\title{
Angewandte
}

Supporting Information

\section{Direct and Versatile Synthesis of Red-Shifted Azobenzenes}

Mickel J. Hansen, Michael M. Lerch, Wiktor Szymanski,* and Ben L. Feringa* anie_201607529_sm_miscellaneous_information.pdf 


\section{Table of Contents}

$\begin{array}{lr}\text { Materials and methods } & \text { S2 }\end{array}$

$\begin{array}{ll}\text { Synthesis } & \text { S3 }\end{array}$

$\begin{array}{ll}\text { Aryldiazonium structures and analyses } & \text { S4 }\end{array}$

$\begin{array}{ll}\text { Optimization table } & \text { S5 }\end{array}$

Synthetic methodology $\quad$ S5

$\begin{array}{ll}\text { UV-Vis spectroscopy and half-life determination } & \text { S13 }\end{array}$

$\begin{array}{ll}\text { Photostationary states (PSS) } & \text { S28 }\end{array}$

${ }^{1} \mathrm{H},{ }^{13} \mathrm{C}-\mathrm{NMR}$ (DMSO-d ${ }_{6}$ ), HR-MS (ESI+) and IR (neat) spectra

References $\quad$ S76 


\section{Materials and methods}

All chemicals for synthesis were obtained from commercial sources and used as received unless stated otherwise.

Thin Layer Chromatography (TLC) was performed using commercial Kieselgel 60, F254 silica gel plates with fluorescence-indicator $\mathrm{UV}_{254}$ (Merck, TLC silica gel $60 F_{254}$ ). For detection of components, UV light at $\lambda=254 \mathrm{~nm}$ or $\lambda=365 \mathrm{~nm}$ was used. Alternatively, oxidative staining using aqueous basic potassium permanganate solution $\left(\mathrm{KMnO}_{4}\right)$ or aqueous acidic cerium phosphomolybdic acid solution (Seebach's stain) was used. Flash chromatography was performed on silica gel (Silicycle Siliaflash P60, 40-63 mm, 230-400 mesh). Drying of solutions was performed with $\mathrm{MgSO}_{4}$ and volatiles were removed with a rotary evaporator.

Nuclear Magnetic Resonance spectra were measured with an Agilent Technologies 400-MR (400/54 Premium Shielded) spectrometer $(400 \mathrm{MHz})$. All spectra were measured at room temperature (22$\left.24{ }^{\circ} \mathrm{C}\right)$. Chemical shifts for ${ }^{1} \mathrm{H}$ - and ${ }^{13} \mathrm{C}-\mathrm{NMR}$ measurements were determined relative to the residual solvent peaks in ppm $\left(\delta_{\mathrm{H}} 7.26\right.$ for $\mathrm{CHCl}_{3}, 2.50$ for DMSO and $2.05 \mathrm{ppm}$ for Acetone, $\delta_{\mathrm{C}} 77.16$ for $\mathrm{CHCl}_{3}$ and 39.52 for DMSO). The following abbreviations are used to indicate signal multiplicity: $\mathrm{s}$, singlet; d, doublet; $t$, triplet; $q$, quartet; $m$, multiplet; brs, broad signal. All ${ }^{13} \mathrm{C}-\mathrm{NMR}$ spectra are ${ }^{1} \mathrm{H}-$ broadband decoupled. High-resolution mass spectrometric measurements were performed on a Thermo scientific LTQ Orbitrap XL with ESI ionization. Melting points were recorded using a Stuart analogue capillary melting point SMP11 apparatus. For spectroscopic measurements, solutions in Uvasol ${ }^{\circledR}$ grade solvents were measured in a $10 \mathrm{~mm}$ quartz cuvette. UV-Vis absorption spectra were recorded on an Agilent 8453 UV-Visible absorption Spectrophotometer. 


\section{Synthesis}

Aryldiazonium salt preparation, method A: ${ }^{[1]}$<smiles>Nc1ccccc1</smiles>

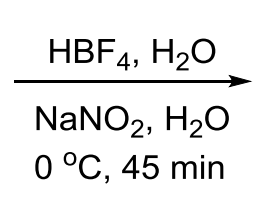<smiles>N#[N+]c1cc#[R]cc1</smiles>

To a solution of aniline $(1.00 \mathrm{mmol})$ in $\mathrm{H}_{2} \mathrm{O}(0.4 \mathrm{~mL})$ was added 50 wt. \% aq. $\mathrm{HBF}_{4}(0.34 \mathrm{~mL})$ and the mixture was stirred while cooling on ice. Subsequently, a solution of $\mathrm{NaNO}_{2}(1.00 \mathrm{mmol}, 69.0 \mathrm{mg})$ in $\mathrm{H}_{2} \mathrm{O}(0.2 \mathrm{~mL})$ was added dropwise. After addition, the reaction mixture was stirred for $45 \mathrm{~min}$ on ice and subsequently filtered over a glass filter. The obtained crystals were washed with $\mathrm{Et}_{2} \mathrm{O}(4 \times 20 \mathrm{~mL})$ and dried under vacuum. The products were stored at room temperature in the dark under $\mathrm{N}_{2}$ atmosphere to prevent degradation.

\section{Aryldiazonium salt preparation, method B: ${ }^{[2]}$}<smiles>[R]c1cccc(N)c1</smiles>

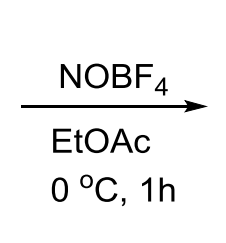<smiles></smiles>

To a stirred solution of aniline $(1.00 \mathrm{mmol})$ in EtOAc $(2.2 \mathrm{~mL})$ at $0{ }^{\circ} \mathrm{C}$ was added $\mathrm{NOBF}_{4}(1.00 \mathrm{mmol}$, $117 \mathrm{mg}$ ) in small portions. After addition, the mixture was stirred at $0{ }^{\circ} \mathrm{C}$ for $1 \mathrm{~h}$ and subsequently filtered over a glass filter. The obtained crystals were washed with $\mathrm{Et}_{2} \mathrm{O}(2 \times 20 \mathrm{~mL})$ and pentane $(2 \mathrm{x}$ $20 \mathrm{~mL}$ ) and dried under vacuum. The products were stored at room temperature in the dark under $\mathrm{N}_{2}$ atmosphere to prevent degradation. 
Table S1. Aryldiazonium structures with ${ }^{1} \mathrm{H}-\mathrm{NMR}$ and IR analysis.

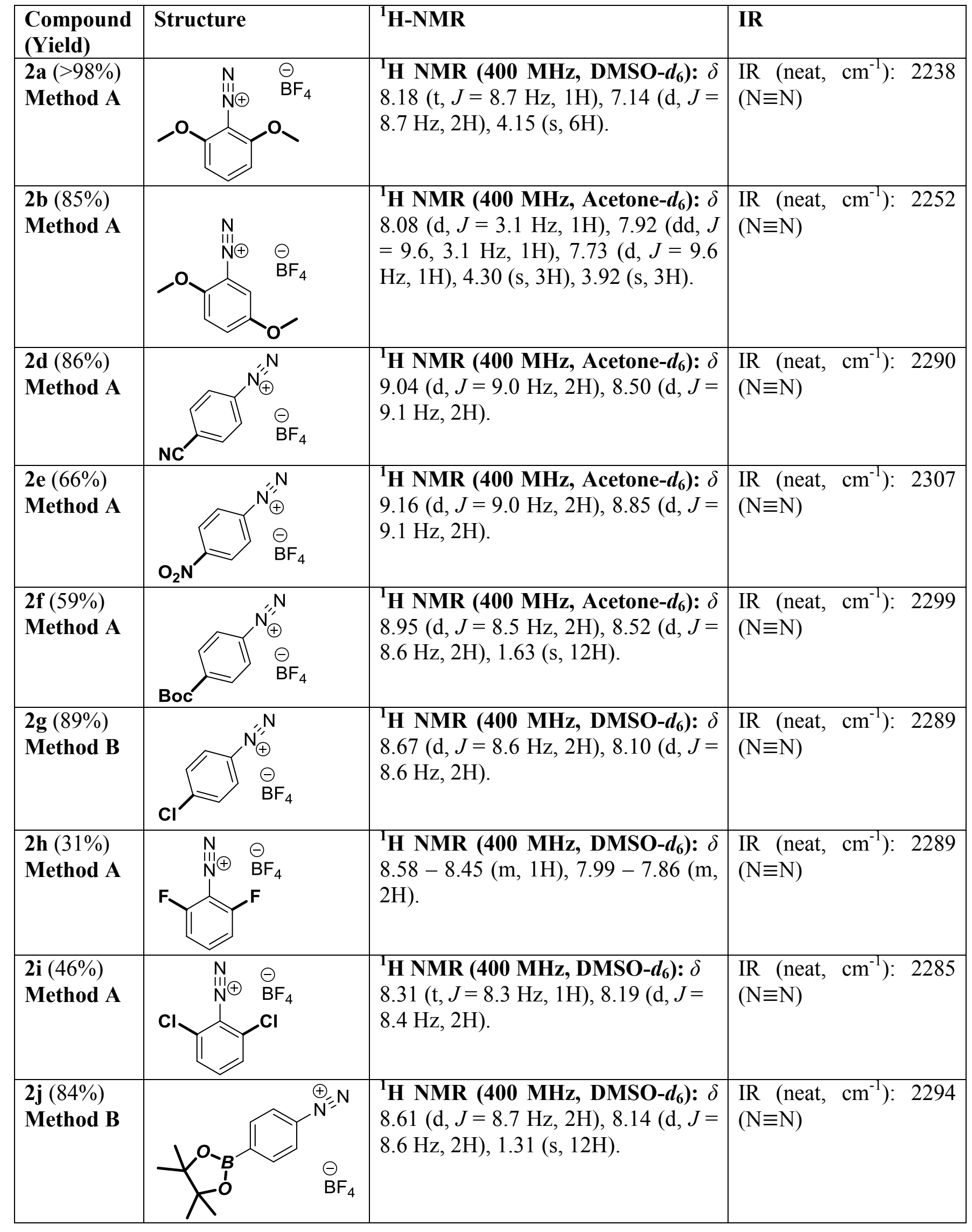


Table S2. Optimization of $\boldsymbol{n}$-butyllithium and 2a concentrations in the novel synthetic methodology leading to the conditions as defined in entry 5.

\begin{tabular}{cccc}
\hline Entry & $\boldsymbol{n}^{\text {-BuLi (equiv) }}$ a $^{\text {a }}$ & 2a (equiv) & Isolated Yield 3a (\%) \\
\hline $\mathbf{1}$ & 1.0 & 1.2 & 71 \\
$\mathbf{2}$ & 1.0 & 1.4 & 64 \\
$\mathbf{3}$ & 1.2 & 1.0 & 48 \\
$\mathbf{4}$ & 1.4 & 1.0 & 23 \\
$\mathbf{5}$ & 1.0 & 1.0 & 86 \\
\hline
\end{tabular}

[a] $1.6 \mathrm{M}$ in hexanes

\section{Synthetic methodology}

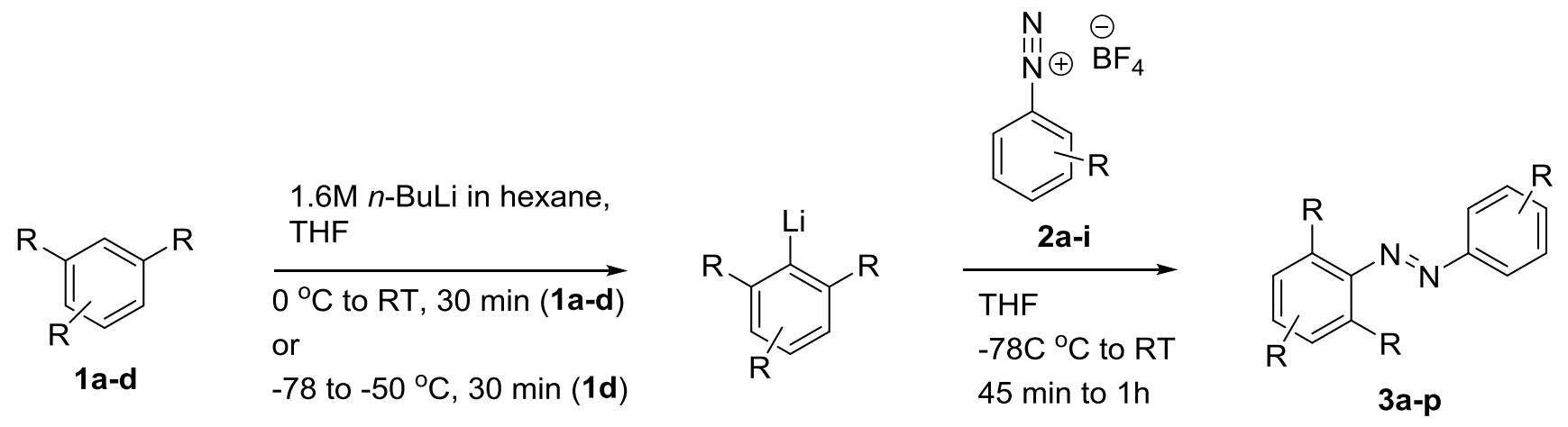

To a stirred solution of $1(1.00 \mathrm{mmol})$ in THF $(1.0 \mathrm{~mL})$ in an oven-dried Schlenk flask under $\mathrm{N}_{2}$ atmosphere at $0{ }^{\circ} \mathrm{C}^{*+}$ was slowly added $1.6 \mathrm{M} n$-BuLi in hexane $(625 \mu \mathrm{L}, 1.00 \mathrm{mmol})$. Subsequently the reaction mixture was allowed to warm up to room temperature over $30 \mathrm{~min}$. This mixture was added to a solution of $2(1.00 \mathrm{mmol})$ in THF $(1.0 \mathrm{~mL})$ in an oven-dried Schlenk flask under $\mathrm{N}_{2}$ atmosphere at $78{ }^{\circ} \mathrm{C}$. The reaction mixture was slowly heated to room temperature over $1 \mathrm{~h}$ and subsequently $2.0 \mathrm{~mL}$ saturated aqueous $\mathrm{NaHCO}_{3}$ solution was added. The resulting solution was extracted with EtOAc $(3 \mathrm{x}$ $20 \mathrm{~mL})$. The combined organic layers were washed with brine and dried $\left(\mathrm{MgSO}_{4}\right)$. Evaporation of all the volatiles yielded the crude product. The product, dissolved in EtOAc, was either precipitated in pentane or subjected to short flash chromatography (Pentane:EtOAc, 95:5 to 3:1) yielding pure azobenzenes.

*Note 1: For compound 1d, a slightly modified lithiation procedure was utilized starting at $-78{ }^{\circ} \mathrm{C}$ instead of $0{ }^{\circ} \mathrm{C}$, and warming to $-50{ }^{\circ} \mathrm{C}$ instead of room temperature in 30 min before addition to a solution of 2 in THF.

${ }^{+}$Note 2: For the synthesis of 3p-r, 1e was kept at $-78{ }^{\circ} \mathrm{C}$ after lithiation and reverse addition of a suspension of $\mathbf{2} \mathbf{a}, \mathbf{j}, \mathbf{h}$ was applied to prevent the formation of side products due to benzyne formation. 


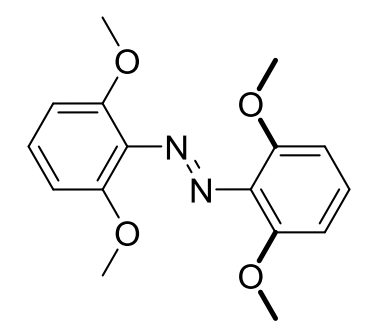

2,2',6,6'-tetra-methoxyazobenzene (3a)

Yield: $86 \%$ (261 mg), brown/yellow crystals

Melting point: $138-140{ }^{\circ} \mathrm{C}$ (Ethyl acetate)

${ }^{1}$ H NMR (400 MHz, DMSO-d $\left.{ }_{6}\right): \delta 7.28$ (t, $\left.J=8.4 \mathrm{~Hz}, 2 \mathrm{H}\right), 6.77(\mathrm{~d}, J=8.4 \mathrm{~Hz}, 4 \mathrm{H}), 3.71(\mathrm{~s}, 12 \mathrm{H})$.

${ }^{13}$ C NMR (100 MHz, DMSO-d $\left.\boldsymbol{d}_{\mathbf{6}}\right): \delta$ 152.0, 133.9, 129.9, 105.8, 56.6.

HR-MS (ESI, [M+H] ${ }^{+}$): Calcd. for $\mathrm{C}_{16} \mathrm{H}_{19} \mathrm{~N}_{2} \mathrm{O}_{4}$ : 303.1339; Found: 303.1344

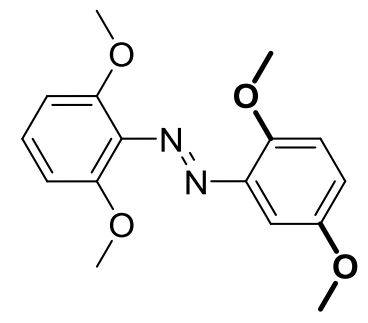

2,2',5',6-tetra-methoxyazobenzene (3b)

Yield: 59\% (180 mg), red oil

${ }^{1}$ H NMR (400 MHz, DMSO- $\left.\boldsymbol{d}_{6}\right): \delta 7.27(\mathrm{t}, J=8.4 \mathrm{~Hz}, 1 \mathrm{H}), 7.18(\mathrm{~d}, J=9.1 \mathrm{~Hz}, 1 \mathrm{H}), 7.11(\mathrm{dd}, J=9.1$, $3.1 \mathrm{~Hz}, 1 \mathrm{H}), 6.89(\mathrm{~d}, J=3.1 \mathrm{~Hz}, 1 \mathrm{H}), 6.78(\mathrm{~d}, J=8.5 \mathrm{~Hz}, 2 \mathrm{H}), 3.85(\mathrm{~s}, 3 \mathrm{H}), 3.72(\mathrm{~s}, 3 \mathrm{H}), 3.71(\mathrm{~s}, 6 \mathrm{H})$.

${ }^{13}$ C NMR (100 MHz, DMSO-d $\left.{ }_{6}\right): \delta$ 162.2, 151.7, 147.2, 133.6, 129.0, 124.5, 114.7, 105.6, 56.5, 56.07.

HR-MS (ESI, [M+H] ${ }^{+}$): Calcd. for $\mathrm{C}_{16} \mathrm{H}_{19} \mathrm{~N}_{2} \mathrm{O}_{4}$ : 303.1339; Found: 303.1344

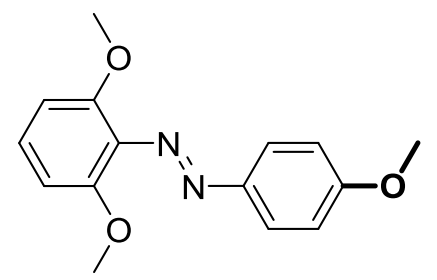

2,6,4'-trimethoxyazobenzene (3c)

Yield: 91\% (248 mg), red oil

${ }^{1}$ H NMR (400 MHz, DMSO-d $\left.\boldsymbol{d}_{6}\right): \delta 7.88-7.64(\mathrm{~m}, 2 \mathrm{H}), 7.25(\mathrm{t}, J=8.4 \mathrm{~Hz}, 1 \mathrm{H}), 7.14-7.01$ (m, 2H), $6.86-6.59(\mathrm{~m}, 2 \mathrm{H}), 3.84(\mathrm{~s}, 3 \mathrm{H}), 3.71(\mathrm{~s}, 6 \mathrm{H})$.

${ }^{13}$ C NMR (100 MHz, DMSO-d $\left.\boldsymbol{d}_{6}\right): \delta$ 162.2, 151.7, 147.2, 133.6, 129.0, 124.5, 114.7, 105.6, 56.5, 56.1.

HR-MS (ESI, [M+H] ${ }^{+}$): Calcd. for $\mathrm{C}_{15} \mathrm{H}_{17} \mathrm{~N}_{2} \mathrm{O}_{4}$ : 273.1233; Found: 273.1238 


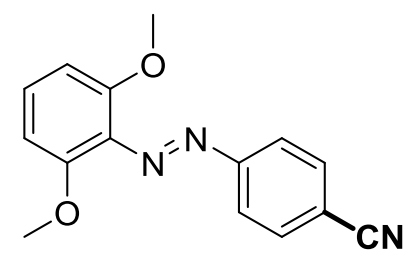

2,6-dimethoxy-4'-cyano-azobenzene (3d)

Yield: $81 \%$ (217 mg), dark red solid

Melting point: $92-93{ }^{\circ} \mathrm{C}$ (Ethyl acetate)

${ }^{1}$ H NMR (400 MHz, DMSO-d $\left.\boldsymbol{d}_{6}\right): \delta 8.03(\mathrm{~d}, J=8.5 \mathrm{~Hz}, 2 \mathrm{H}), 7.86(\mathrm{~d}, J=8.5 \mathrm{~Hz}, 2 \mathrm{H}), 7.37(\mathrm{t}, J=8.5$ $\mathrm{Hz}, 1 \mathrm{H}), 6.82(\mathrm{~d}, J=8.5 \mathrm{~Hz}, 2 \mathrm{H}), 3.76(\mathrm{~s}, 6 \mathrm{H})$.

${ }^{13}$ C NMR (100 MHz, DMSO-d $\left.\mathbf{d}_{6}\right): \delta$ 155.0, 152.5, 134.2, 133.0, 131.6, 123.1, 118.9, 113.4, 105.6, 56.6.

HR-MS (ESI, $[\mathbf{M}+\mathbf{H}]^{+}$): Calcd. for $\mathrm{C}_{15} \mathrm{H}_{14} \mathrm{~N}_{3} \mathrm{O}_{2}$ : 268.1080; Found: 268.1085<smiles>COc1cccc(OC)c1N=Nc1ccc([N+](=O)[O-])cc1</smiles>

2,6-dimethoxy-4'-nitro-azobenzene (3e)

Yield: $67 \%$ (193 mg), red oil

${ }^{1}$ H NMR (400 MHz, DMSO-d $\left.\mathbf{d}_{\mathbf{6}}\right): \delta 8.38(\mathrm{~d}, J=9.0 \mathrm{~Hz}, 2 \mathrm{H}), 7.92(\mathrm{~d}, J=9.0 \mathrm{~Hz}, 2 \mathrm{H}), 7.38(\mathrm{t}, J=8.5$ $\mathrm{Hz}, 1 \mathrm{H}), 6.82(\mathrm{~d}, J=8.5 \mathrm{~Hz}, 2 \mathrm{H}), 3.78(\mathrm{~s}, 6 \mathrm{H})$.

${ }^{13}$ C NMR (100 MHz, DMSO-d $\left.\mathbf{d}\right): \delta$ 156.3, 152.7, 148.6, 133.0, 132.0, 125.4, 123.3, 105.6, 56.7.

HR-MS (ESI, $[\mathbf{M}+\mathbf{H}]^{+}$): Calcd. for $\mathrm{C}_{14} \mathrm{H}_{14} \mathrm{~N}_{3} \mathrm{O}_{4}$ : 288.0978; Found: 288.0984<smiles>COc1cccc(OC)c1N=Nc1ccc(C(=O)OC(C)(C)C)cc1</smiles>

2,6-dimethoxy-4'-Boc-azobenzene (3f)

Yield: 45\% (154 mg), red oil

${ }^{1}$ H NMR (400 MHz, DMSO-d $\left.\mathbf{d}_{6}\right): \delta 8.09(\mathrm{~d}, J=8.3 \mathrm{~Hz}, 2 \mathrm{H}), 7.84(\mathrm{~d}, J=8.3 \mathrm{~Hz}, 2 \mathrm{H}), 7.34(\mathrm{t}, J=8.4$ $\mathrm{Hz}, 1 \mathrm{H}), 6.81(\mathrm{~d}, J=8.5 \mathrm{~Hz}, 2 \mathrm{H}), 3.77(\mathrm{~s}, 6 \mathrm{H}), 1.57(\mathrm{~s}, 9 \mathrm{H})$.

${ }^{13}$ C NMR (100 MHz, DMSO-d $\left.\boldsymbol{d}_{6}\right): \delta 164.3,154.9,151.8,132.9,130.2,122.03,105.13,81.13,56.14$, 27.71 .

HR-MS (ESI, [M+H] ${ }^{+}$): Calcd. for $\mathrm{C}_{19} \mathrm{H}_{23} \mathrm{~N}_{2} \mathrm{O}_{4}$ : 343.1652; Found: 343.1658 


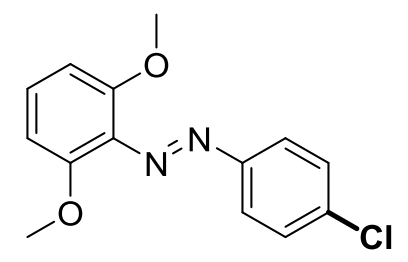

2,6-dimethoxy-4'-chloro-azobenzene (3g)

Yield: $77 \%$ (213 mg), orange solid

Melting point: $77-78^{\circ} \mathrm{C}$ (Ethyl acetate)

${ }^{1}$ H NMR (400 MHz, DMSO-d $\left.\boldsymbol{d}_{6}\right): \delta 7.77(\mathrm{~d}, J=8.7 \mathrm{~Hz}, 2 \mathrm{H}), 7.61(\mathrm{~d}, J=8.8 \mathrm{~Hz}, 2 \mathrm{H}), 7.31(\mathrm{t}, J=8.4$ $\mathrm{Hz}, 1 \mathrm{H}), 6.79(\mathrm{~d}, J=8.5 \mathrm{~Hz}, 2 \mathrm{H}), 3.74(\mathrm{~s}, 6 \mathrm{H})$.

${ }^{13}$ C NMR (100 MHz, DMSO-d $\left.\boldsymbol{d}_{6}\right)$ : $\delta$ 152.1, 151.5, 136.2, 133.2, 130.4, 129.8, 124.2, 105.6, 56.6.

HR-MS (ESI, [M+H] ${ }^{+}$): Calcd. for $\mathrm{C}_{14} \mathrm{H}_{14} \mathrm{ClN}_{2} \mathrm{O}_{2}$ : 277.0738; Found: 277.0743

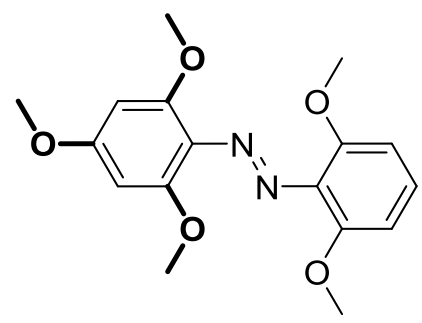

2,2',6,6',4-pentamethoxyazobenzene (3h)

Yield: $81 \%$ (269 mg), red oil

${ }^{1}$ H NMR (400 MHz, DMSO-d d $_{\text {) }} \delta 7.21(\mathrm{t}, J=8.4 \mathrm{~Hz}, 1 \mathrm{H}), 6.74(\mathrm{~d}, J=8.4 \mathrm{~Hz}, 2 \mathrm{H}), 6.33(\mathrm{~s}, 2 \mathrm{H}), 3.84$ $(\mathrm{s}, 3 \mathrm{H}), 3.73(\mathrm{~s}, 6 \mathrm{H}), 3.68(\mathrm{~s}, 6 \mathrm{H})$.

${ }^{13}$ C NMR (100 MHz, DMSO-d $\left.\boldsymbol{d}_{6}\right): \delta 162.0,154.5,151.5,134.8,128.6,127.7,105.8,92.0,56.5,56.5$, 56.0 .

HR-MS (ESI, [M+H] ${ }^{+}$): Calcd. for $\mathrm{C}_{17} \mathrm{H}_{21} \mathrm{~N}_{2} \mathrm{O}_{5}$ : 333.1445; Found: 333.1450

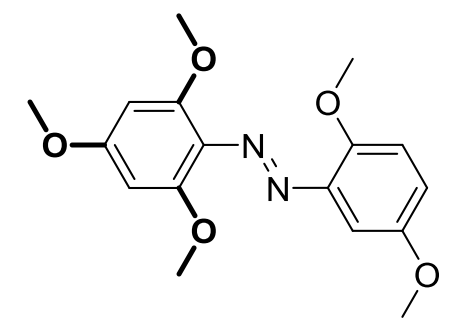

2,2',4,5',6-pentamethoxyazobenzene (3i)

Yield: 41\% (137 mg), red oil

${ }^{1}$ H NMR (400 MHz, DMSO-d $\left.{ }_{6}\right): \delta 7.11(\mathrm{~d}, J=9.0 \mathrm{~Hz}, 1 \mathrm{H}), 7.02(\mathrm{dd}, J=9.0,3.2 \mathrm{~Hz}, 1 \mathrm{H}), 6.86(\mathrm{~d}, J=$ $3.2 \mathrm{~Hz}, 1 \mathrm{H}), 6.35(\mathrm{~s}, 2 \mathrm{H}), 3.85(\mathrm{~d}, J=4.5 \mathrm{~Hz}, 6 \mathrm{H}), 3.76(\mathrm{~s}, 6 \mathrm{H}), 3.72(\mathrm{~s}, 3 \mathrm{H})$. 
${ }^{13}$ C NMR (100 MHz, DMSO-d $\left.\mathbf{6}\right): \delta$ 161.8, 154.2, 153.8, 151.0, 143.7, 128.1, 117.6, 115.7, 101.1, 92.1, $57.5,56.6,55.9,55.9$.

HR-MS (ESI, $[\mathbf{M}+\mathbf{H}]^{+}$): Calcd. for $\mathrm{C}_{17} \mathrm{H}_{21} \mathrm{~N}_{2} \mathrm{O}_{5}$ : 333.1445; Found: 333.1450

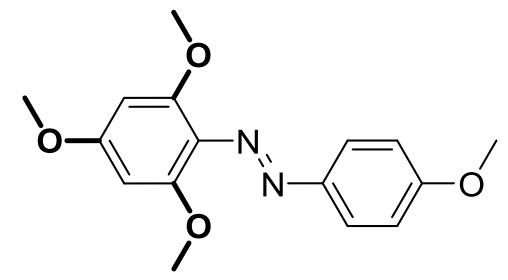

2,4,4',6-tetramethoxyazobenzene (3j)

Yield: $86 \%$ (260 mg), red oil

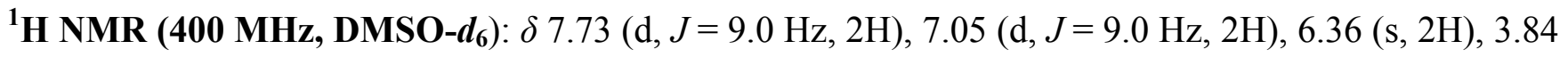
(s, 3H), $3.82(\mathrm{~s}, 3 \mathrm{H}), 3.77(\mathrm{~s}, 6 \mathrm{H})$.

${ }^{13}$ C NMR (100 MHz, DMSO-d $\left.\boldsymbol{d}_{6}\right): \delta$ 161.5, 161.3, 154.1, 147.8, 127.5, 124.1, 114.6, 92.1, 56.5, 55.8.

HR-MS (ESI, [M+H] ${ }^{+}$): Calcd. for $\mathrm{C}_{16} \mathrm{H}_{19} \mathrm{~N}_{2} \mathrm{O}_{4}$ : 303.1339; Found: 303.1343

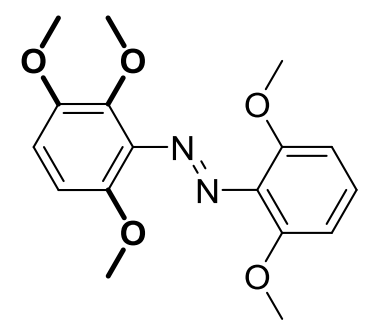

2,2',3,6,6'-pentamethoxyazobenzene (3k)

Yield: 76\% (253 mg), red oil

${ }^{1}$ H NMR (400 MHz, DMSO-d $\left.\mathbf{d}_{6}\right): \delta 7.31(\mathrm{td}, J=8.4,1.9 \mathrm{~Hz}, 1 \mathrm{H}), 6.98(\mathrm{dd}, J=9.1,1.9 \mathrm{~Hz}, 1 \mathrm{H}), 6.88-$ $6.73(\mathrm{~m}, 3 \mathrm{H}), 3.78(\mathrm{~d}, J=1.9 \mathrm{~Hz}, 3 \mathrm{H}), 3.74(\mathrm{~d}, J=1.9 \mathrm{~Hz}, 6 \mathrm{H}), 3.70(\mathrm{~d}, J=1.9 \mathrm{~Hz}, 3 \mathrm{H}), 3.58(\mathrm{~d}, J=$ $1.9 \mathrm{~Hz}, 3 \mathrm{H})$.

${ }^{13}$ C NMR (100 MHz, DMSO-d $)$ : $\delta$ 152.2, 147.7, 146.6, 139.8, 133.6, 130.4, 112.6, 107.9, 105.7, 61.6, $56.9,56.8,56.6$.

HR-MS (ESI, [M+H] ${ }^{+}$): Calcd. for $\mathrm{C}_{17} \mathrm{H}_{21} \mathrm{~N}_{2} \mathrm{O}_{5}$ : 333.1445; Found: 333.1449

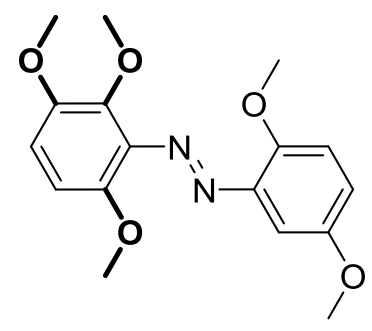

2,2',3,5',6-pentamethoxyazobenzene (31)

Yield: $58 \%$ (193 mg), red oil 
${ }^{1}$ H NMR (400 MHz, DMSO-d d $_{\text {): }} \delta 7.21(\mathrm{~d}, J=9.1 \mathrm{~Hz}, 1 \mathrm{H}), 7.14(\mathrm{dd}, J=9.1,3.1 \mathrm{~Hz}, 1 \mathrm{H}), 6.99(\mathrm{~d}, J=$ $9.1 \mathrm{~Hz}, 1 \mathrm{H}), 6.94(\mathrm{~d}, J=3.1 \mathrm{~Hz}, 1 \mathrm{H}), 6.83(\mathrm{~d}, J=9.2 \mathrm{~Hz}, 1 \mathrm{H}), 3.86(\mathrm{~s}, 3 \mathrm{H}), 3.78(\mathrm{~s}, 3 \mathrm{H}), 3.73(\mathrm{~s}, 6 \mathrm{H})$, $3.70(\mathrm{~s}, 3 \mathrm{H}), 3.63(\mathrm{~s}, 3 \mathrm{H})$.

${ }^{13}$ C NMR (100 MHz, DMSO-d d $_{\text {): }} \delta$ 153.7, 151.9, 147.6, 146.6, 142.3, 140.1, 139.6, 119.7, 115.6, $112.6,107.7,100.4,62.1,57.1,56.8,56.7,56.0$.

HR-MS (ESI, [M+H] ${ }^{+}$): Calcd. for $\mathrm{C}_{17} \mathrm{H}_{21} \mathrm{~N}_{2} \mathrm{O}_{5}: 333.1445$; Found: 333.1450

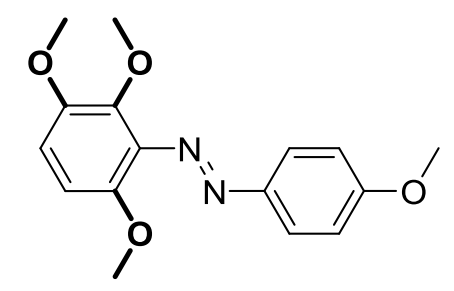

2,3,4',6-tetramethoxyazobenzene $(\mathbf{3 m})$

Yield: 64\% (193 mg), red oil

${ }^{1}$ H NMR (400 MHz, DMSO-d N $): \delta 7.80(\mathrm{~d}, J=9.0 \mathrm{~Hz}, 2 \mathrm{H}), 7.11(\mathrm{~d}, J=9.0 \mathrm{~Hz}, 2 \mathrm{H}), 6.97(\mathrm{~d}, J=9.2$ $\mathrm{Hz}, 1 \mathrm{H}), 6.82(\mathrm{~d}, J=9.2 \mathrm{~Hz}, 1 \mathrm{H}), 3.85(\mathrm{~s}, 3 \mathrm{H}), 3.78(\mathrm{~s}, 3 \mathrm{H}), 3.69(\mathrm{~s}, 3 \mathrm{H}), 3.63(\mathrm{~s}, 3 \mathrm{H})$.

${ }^{13}$ C NMR (100 MHz, DMSO-d $\left.\mathbf{6}\right): \delta 162.5,147.6,147.1,146.4,140.2,139.3,124.7,114.9,112.2$, $107.7,61.8,56.8,56.7,56.1$.

HR-MS (ESI, [M+H] ${ }^{+}$): Calcd. for $\mathrm{C}_{16} \mathrm{H}_{19} \mathrm{~N}_{2} \mathrm{O}_{4}$ : 303.1339; Found: 303.1344

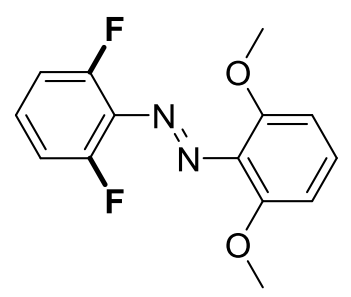

2,6-difluor-2',6'-dimethoxyazobenzene (3n)

Yield: $82 \%(228 \mathrm{mg})$, red solid

Melting point: $128-129^{\circ} \mathrm{C}$ (Ethyl acetate)

${ }^{1}$ H NMR (400 MHz, DMSO-d $\left.\mathbf{d}_{6}\right): \delta 7.57-7.44(\mathrm{~m}, 1 \mathrm{H}), 7.37(\mathrm{t}, J=8.5 \mathrm{~Hz}, 1 \mathrm{H}), 7.28(\mathrm{td}, J=9.0,2.1$ $\mathrm{Hz}, 2 \mathrm{H}), 6.82(\mathrm{~d}, J=8.5 \mathrm{~Hz}, 2 \mathrm{H}), 3.76(\mathrm{~s}, 6 \mathrm{H})$.

${ }^{13}$ C NMR (100 MHz, DMSO-d d $_{\text {): } \delta} 155.9$ (d, $\left.J=4.7 \mathrm{~Hz}\right), 153.3$ (d, $\left.J=4.6 \mathrm{~Hz}\right), 152.3$, $133.5,131.7$, $131.5(\mathrm{t}, J=10.3 \mathrm{~Hz}), 113.9-112.8(\mathrm{~m}), 105.6,56.7$.

${ }^{19}$ F NMR (376 MHz, DMSO-d $\left.\boldsymbol{d}_{6}\right): \delta-123.88(\mathrm{~m})$.

HR-MS (ESI, [M+H] ${ }^{+}$): Calcd. for $\mathrm{C}_{14} \mathrm{H}_{13} \mathrm{~F}_{2} \mathrm{~N}_{2} \mathrm{O}_{2}$ : 279.0939; Found: 279.0944 


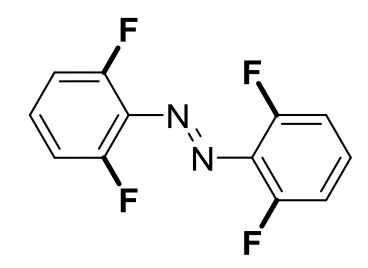

2,2',6,6'-tetrafluoroazobenzene (3o)

Yield: $77 \%$ (196 mg), red oil

${ }^{1}$ H NMR (400 MHz, DMSO-d N $): \delta .68-7.52(\mathrm{~m}, 2 \mathrm{H}), 7.34(\mathrm{t}, J=9.2 \mathrm{~Hz}, 4 \mathrm{H})$.

${ }^{13}$ C NMR (100 MHz, DMSO-d $): \delta 156.3$ (d, $\left.J=4.0 \mathrm{~Hz}\right), 153.7(\mathrm{~d}, J=4.3 \mathrm{~Hz}), 133.6(\mathrm{t}, J=10.7 \mathrm{~Hz})$, $113.6(\mathrm{dd}, J=19.9,3.6 \mathrm{~Hz})$.

${ }^{19}$ F NMR (376 MHz, DMSO-d $\left.\boldsymbol{d}_{6}\right): \delta-122.01(\mathrm{dd}, J=10.1,5.9 \mathrm{~Hz})$.

HR-MS (ESI, [M+H] ${ }^{+}$): Calcd. for $\mathrm{C}_{12} \mathrm{H}_{7} \mathrm{~F}_{4} \mathrm{~N}_{2}$ : 255.0539; Found: 255.0544

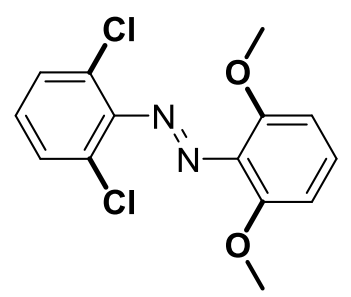

2,6-dichloro-2',6'-dimethoxyazobenzene (3p)

Yield: $91 \%$ (284 mg), red solid (Ethyl acetate)

Melting point: $79-82^{\circ} \mathrm{C}$

${ }^{1}$ H NMR (400 MHz, DMSO-d d $_{\text {): } \delta} 7.59$ (d, $\left.J=8.1 \mathrm{~Hz}, 2 \mathrm{H}\right), 7.49-7.26(\mathrm{~m}, 2 \mathrm{H}), 6.84(\mathrm{~d}, J=8.5 \mathrm{~Hz}$, 2H), $3.79(\mathrm{~s}, 6 \mathrm{H})$.

${ }^{13}$ C NMR (100 MHz, DMSO-d $\left.\boldsymbol{d}_{6}\right): \delta$ 153.1, 148.9, 132.6, 132.3, 129.77, 129.6, 125.7, 105.6, 56.7.

HR-MS (ESI, [M+H] ${ }^{+}$): Calcd. for $\mathrm{C}_{14} \mathrm{H}_{12} \mathrm{Cl}_{2} \mathrm{~N}_{2} \mathrm{O}_{2}$ : 311.03486 ; Found: 311.03447

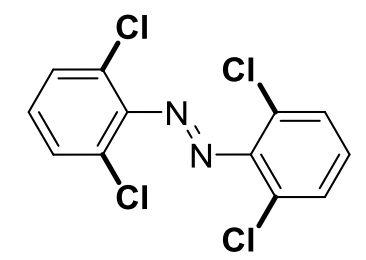

2,6,2',6'-tetrachloroazobenzene (3q)

Yield: $92 \%$ (296 mg), bright orange solid

Melting point: $120-121^{\circ} \mathrm{C}$ (Ethyl acetate)

${ }^{1}$ H NMR (400 MHz, DMSO-d $\left.d_{6}\right): \delta 7.71(\mathrm{~d}, 4 \mathrm{H}), 7.51(\mathrm{t}, J=7.7,2 \mathrm{H})$

${ }^{13}$ C NMR (100 MHz, DMSO- $\left.\boldsymbol{d}_{6}\right): \delta$ 146.9, 131.7, 130.4, 126.5 . 
HR-MS (ESI, $[\mathbf{M}+\mathbf{H}]^{+}$): Calcd. for $\mathrm{C}_{12} \mathrm{H}_{6} \mathrm{Cl}_{4} \mathrm{~N}_{2}$ : 320.9328; Found: 320.9324

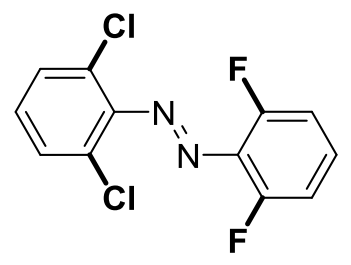

2,6-dichloro-2',6'-difluoroazobenzene (3r)

Yield: $89 \%$ (256 mg), dark red solid

Melting point: $66-69^{\circ} \mathrm{C}$ (Ethyl acetate)

${ }^{1}$ H NMR (400 MHz, DMSO-d $\left.\boldsymbol{d}_{6}\right): \delta 7.74-7.67(\mathrm{~m}, 1 \mathrm{H}), 7.66(\mathrm{~d}, J=8.1 \mathrm{~Hz}, 2 \mathrm{H}), 7.46(\mathrm{t}, J=8.1 \mathrm{~Hz}$, $1 \mathrm{H}), 7.39(\mathrm{t}, J=9.0 \mathrm{~Hz}, 2 \mathrm{H})$.

${ }^{13}$ C NMR (100 MHz, DMSO- $\left.\boldsymbol{d}_{6}\right): \delta 156.5(\mathrm{~d}, J=4.1 \mathrm{~Hz}), 153.9(\mathrm{~d}, J=4.1 \mathrm{~Hz}), 148.1,134.4(\mathrm{t}, J=$ $10.6 \mathrm{~Hz}), 130.9$, $130.1,125.7,113.8$ (dd, $J=19.7,3.6 \mathrm{~Hz})$.

${ }^{19}$ F NMR (376 MHz, DMSO- $\left.\boldsymbol{d}_{6}\right): \delta-121.29(\mathrm{dd}, J=10.1,6.2 \mathrm{~Hz})$.

HR-MS (ESI, [M+H] ${ }^{+}$): Calcd. for $\mathrm{C}_{12} \mathrm{H}_{6} \mathrm{Cl}_{4} \mathrm{~N}_{2}$ : 286.9948; Found: 286.9949

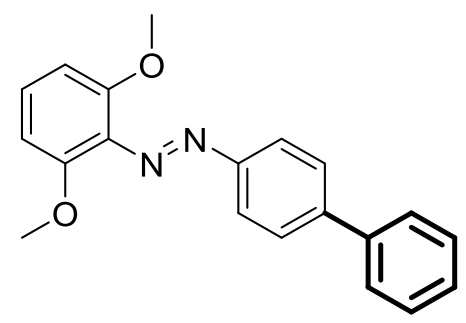

2,6-dimethoxy-4'-phenylazobenzene (3s)

After reaction of 1,3 dimethoxybenzene with para-boronic ester aryldiazonium salt as described in the general methodology, direct addition of a solution of bromobenzene (172 mg, $1.10 \mathrm{mmol}), \mathrm{Pd}\left(\mathrm{PPh}_{3}\right)_{4}$ $(57.0 \mathrm{mg}, 0.05 \mathrm{mmol}), 1 \mathrm{M}$ aqueous $\mathrm{K}_{2} \mathrm{CO}_{3}(2.0 \mathrm{~mL})$ and dioxane $(2.0 \mathrm{~mL})$ to the reaction mixture, led to a biphasic mixture which was heated to reflux under $\mathrm{N}_{2}$ atmosphere for $16 \mathrm{~h}$. After cooling to room temperature $\mathrm{H}_{2} \mathrm{O}$ was added and the mixture was extracted with EtOAc $(3 \times 20 \mathrm{~mL})$. The combined organic layers were washed with brine and dried $\left(\mathrm{MgSO}_{4}\right)$ and the pure product was obtained after purification by flash chromatography (Pentane:EtOAc 9:1 to 3:1).

Yield: 41\% (130 mg), light orange oil

${ }^{1}$ H NMR (400 MHz, DMSO-d $\left.\mathbf{d}_{6}\right): \delta 7.86(\mathrm{~s}, 4 \mathrm{H}), 7.75(\mathrm{~d}, J=7.1 \mathrm{~Hz}, 2 \mathrm{H}), 7.50(\mathrm{t}, J=7.5 \mathrm{~Hz}, 2 \mathrm{H}), 7.41$ (t, $J=7.3 \mathrm{~Hz}, 1 \mathrm{H}), 7.30$ (t, $J=8.5 \mathrm{~Hz}, 1 \mathrm{H}), 6.80(\mathrm{~d}, J=8.5 \mathrm{~Hz}, 2 \mathrm{H}), 3.74(\mathrm{~s}, 6 \mathrm{H})$.

${ }^{13}$ C NMR (100 MHz, DMSO-d $\left.\boldsymbol{d}_{\mathbf{6}}\right): \delta 152.1,151.9,143.2,139.5,133.5,129.9,129.5,128.6,127.9$, $127.3,123.2,105.6,56.6$.

HR-MS (ESI, [M+Na] ${ }^{+}$): Calcd. for $\mathrm{C}_{20} \mathrm{H}_{18} \mathrm{~N}_{2} \mathrm{O}_{2}: 341.1260$; Found: 341.1265 


\section{UV-Vis Spectroscopy}

Solutions were prepared in DMSO or as a mixture of four buffer salts (Bis-TRIS, TRIS, MES, sodium acetate, $25 \mathrm{mM}$ each) to ensure the $\mathrm{pH}$ could be easily adjusted between 2 and 10 by addition of small quantities of concentrated hydrochloric acid and sodium hydroxide. All the compounds were dissolved in DMSO and thermally adapted by heating at $150{ }^{\circ} \mathrm{C}$ for $2 \mathrm{~min}$. The measurements were taken with final concentrations of $20 \mu \mathrm{M}$ in DMSO or $1 \mathrm{vol} \%$ DMSO in buffer.

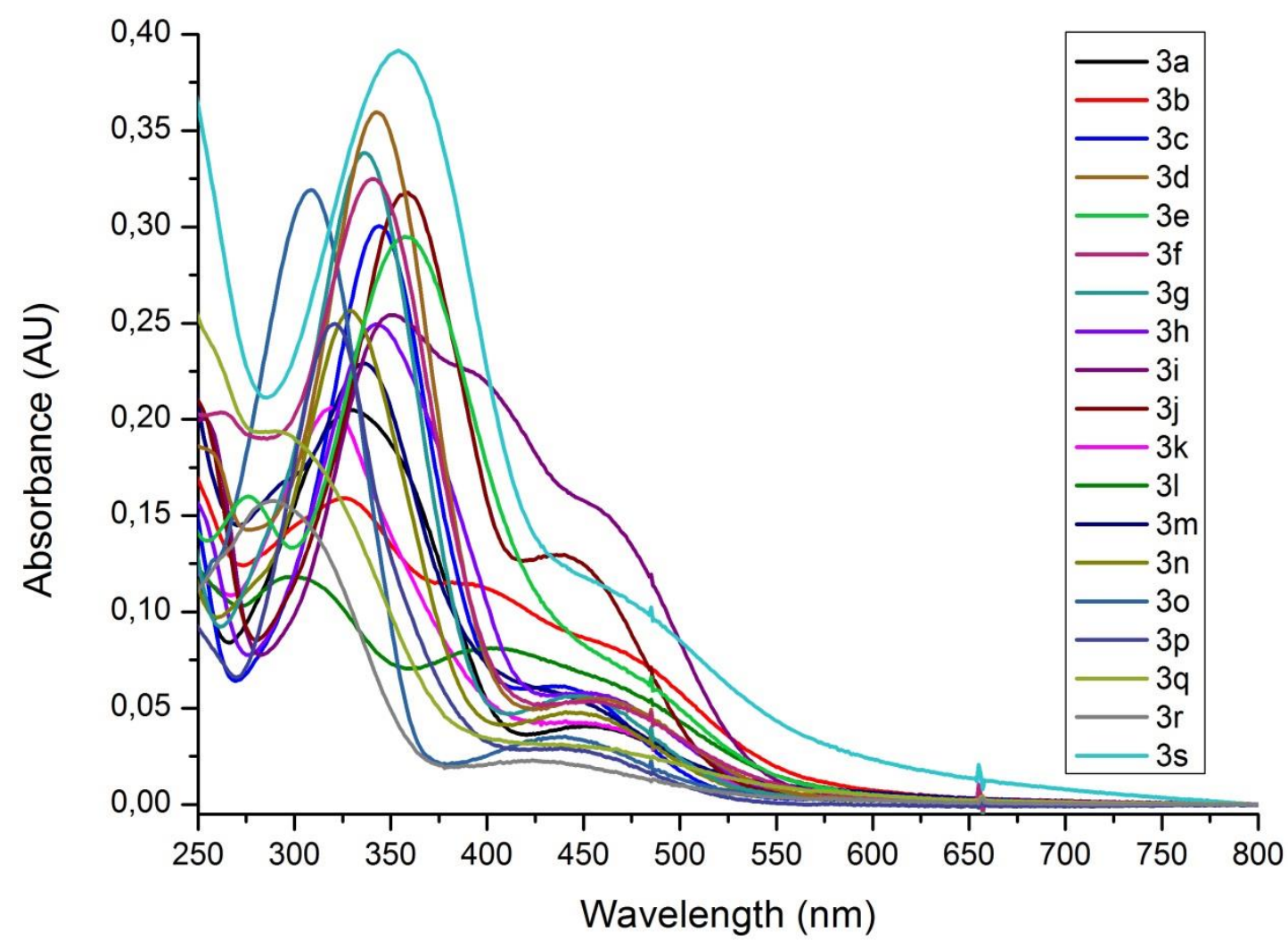

Figure S1. Absorption spectra of the newly synthesized azobenzene derivatives $\mathbf{3 a}-\mathbf{3 p}(20 \mu \mathrm{M}$ in buffer with $1 \mathrm{vol} \%$ DMSO at $\mathrm{pH}=7$ ) 


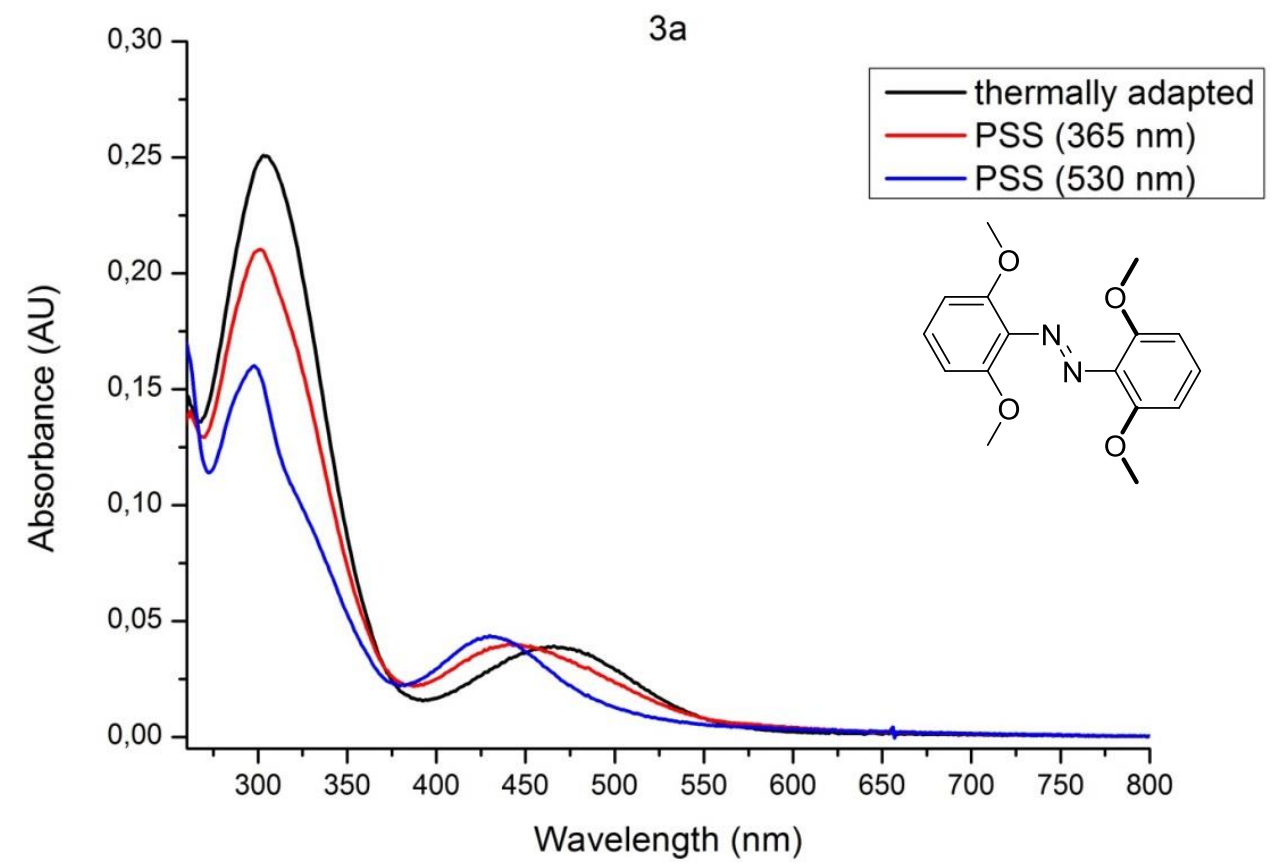

Figure S2. Absorption spectra for the photoisomerization of compound 3a (DMSO, $37^{\circ} \mathrm{C}, 20 \mu \mathrm{M}$ ). Photostationary states after thermal adaptation, $365 \mathrm{~nm}$ irradiation and $530 \mathrm{~nm}$ irradiation were obtained.

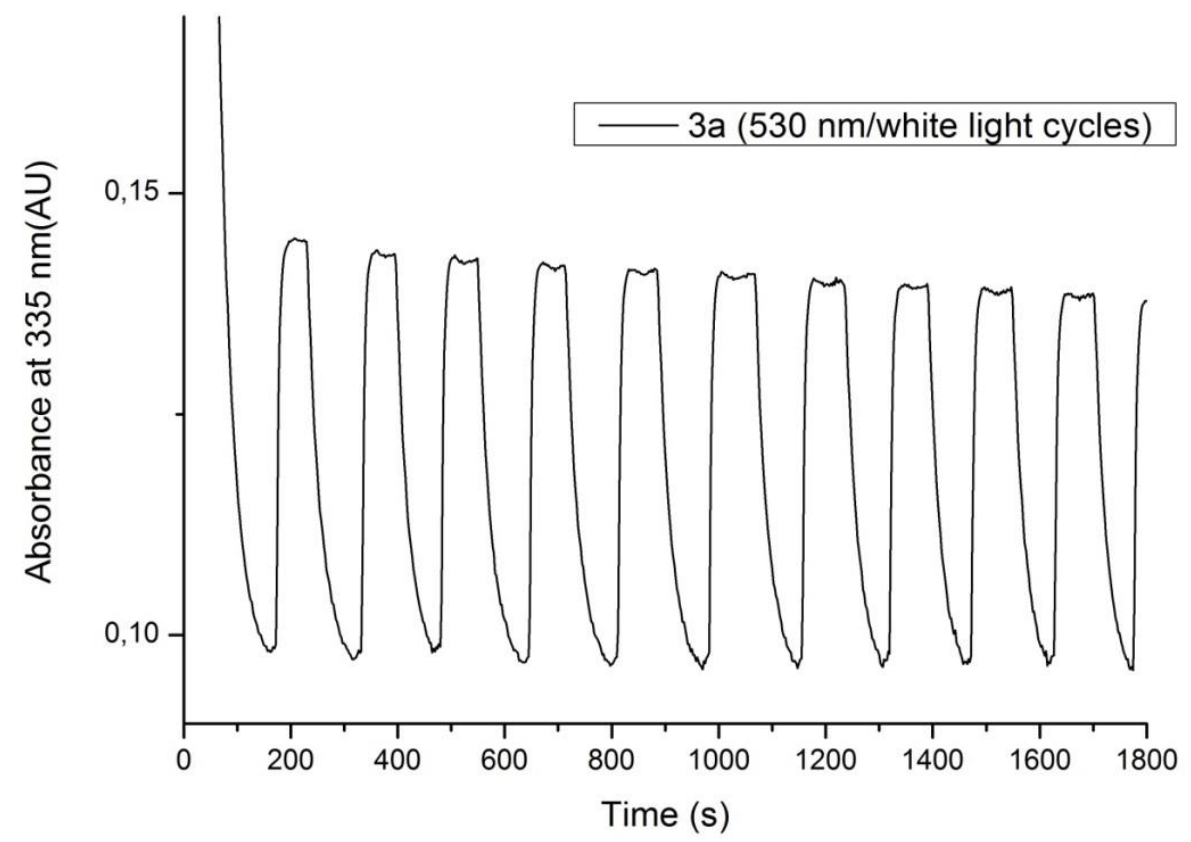

Figure S3. Reversible photosisomerization of 3a ( $37^{\circ} \mathrm{C}$, Buffer with 1 vol\% DMSO at $\mathrm{pH}=7,20 \mu \mathrm{M}$ ) in the presence of $10 \mathrm{mM}$ glutathione (GSH). Ten cycles of $530 \mathrm{~nm} /$ white light irradiation could be performed without the observation of significant fatigue or reduction. 


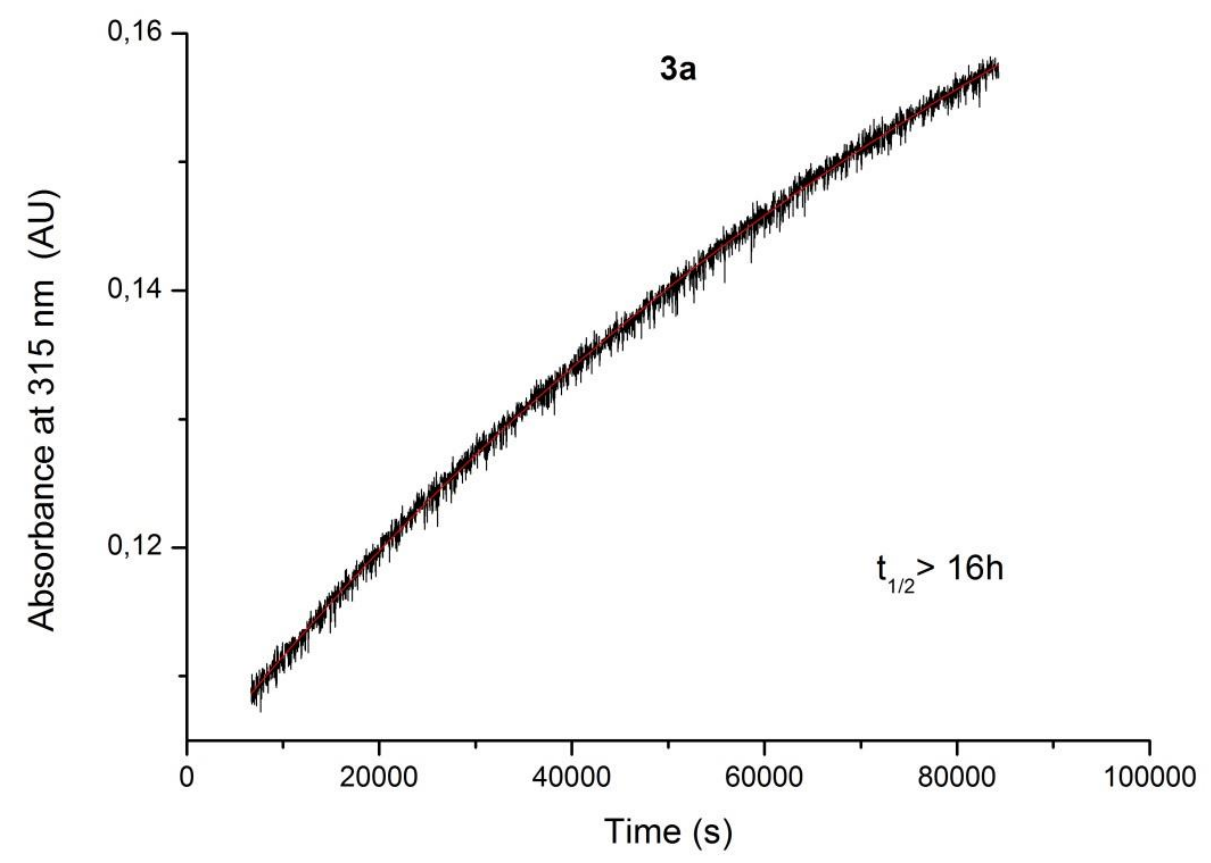

Figure S4. Determination of half-life for 3a at $37{ }^{\circ} \mathrm{C}$ in DMSO. First, PSS was reached upon $530 \mathrm{~nm}$ irradiation, after which the absorption was measured at $\lambda_{\max }=315 \mathrm{~nm}$. Line presents the fitting with single exponential process. 


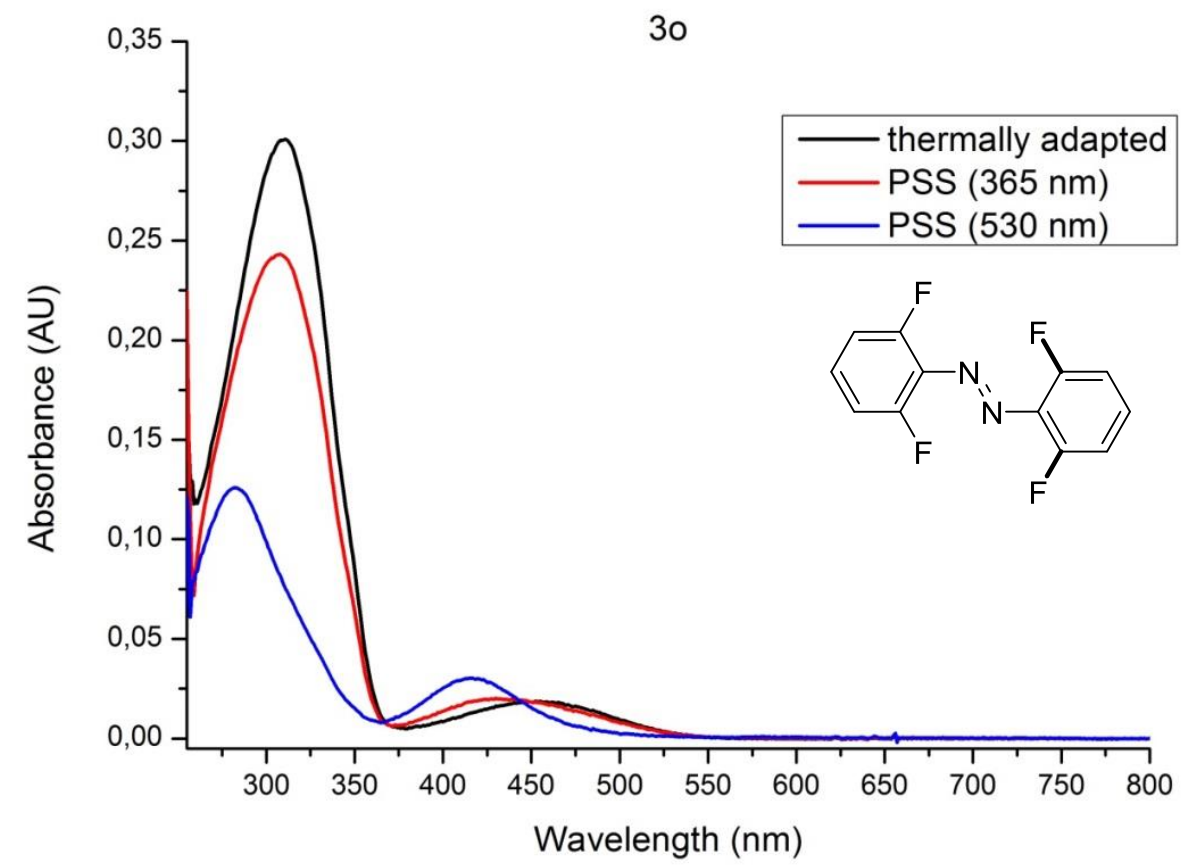

Figure S5. Absorption spectra for the photoisomerization of compound 3o (DMSO, $37{ }^{\circ} \mathrm{C}, 20 \mu \mathrm{M}$ ). Photostationary states after thermal adaptation, $365 \mathrm{~nm}$ irradiation and $530 \mathrm{~nm}$ irradiation were obtained.

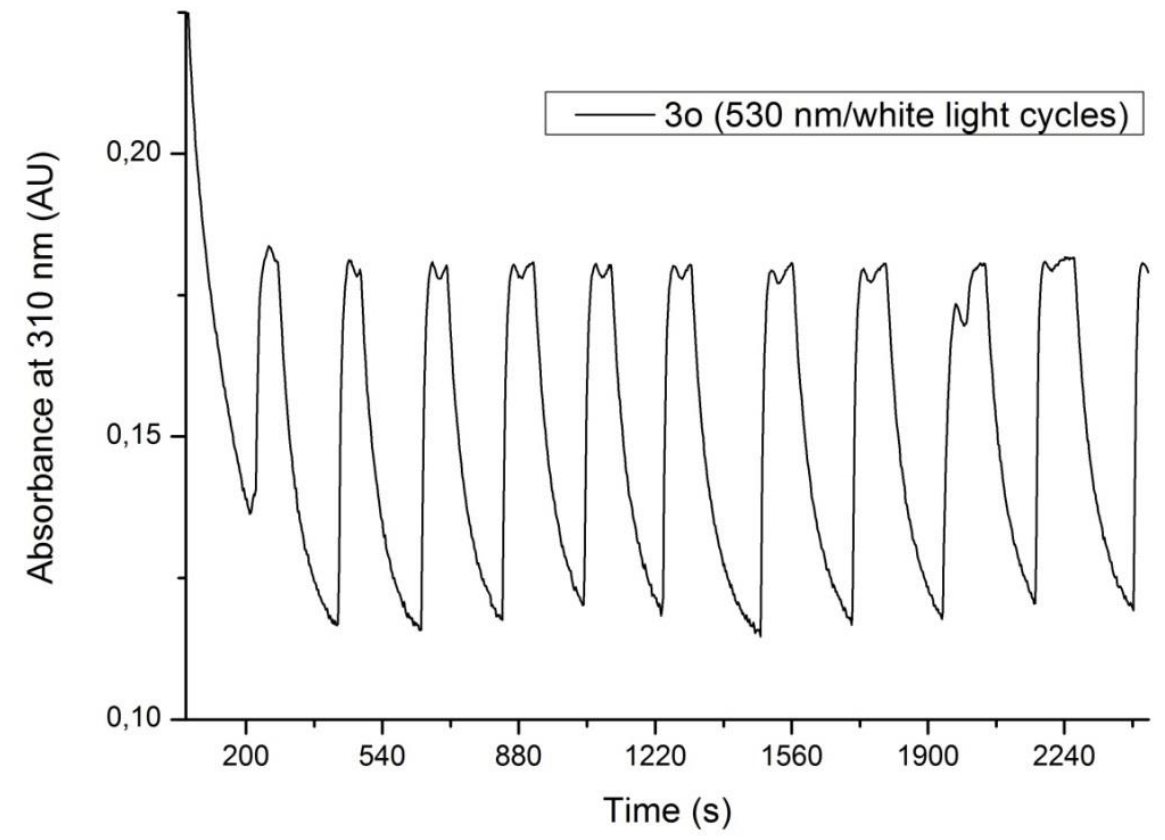

Figure S6. Reversible photosisomerization of 30 ( $37^{\circ} \mathrm{C}$, Buffer with 1 vol\% DMSO at $\mathrm{pH}=7,20 \mu \mathrm{M}$ ) in the presence of $10 \mathrm{mM}$ glutathione $(\mathrm{GSH})$. Ten cycles of $530 \mathrm{~nm} /$ white light irradiation could be performed without the observation of significant fatigue or reduction. 


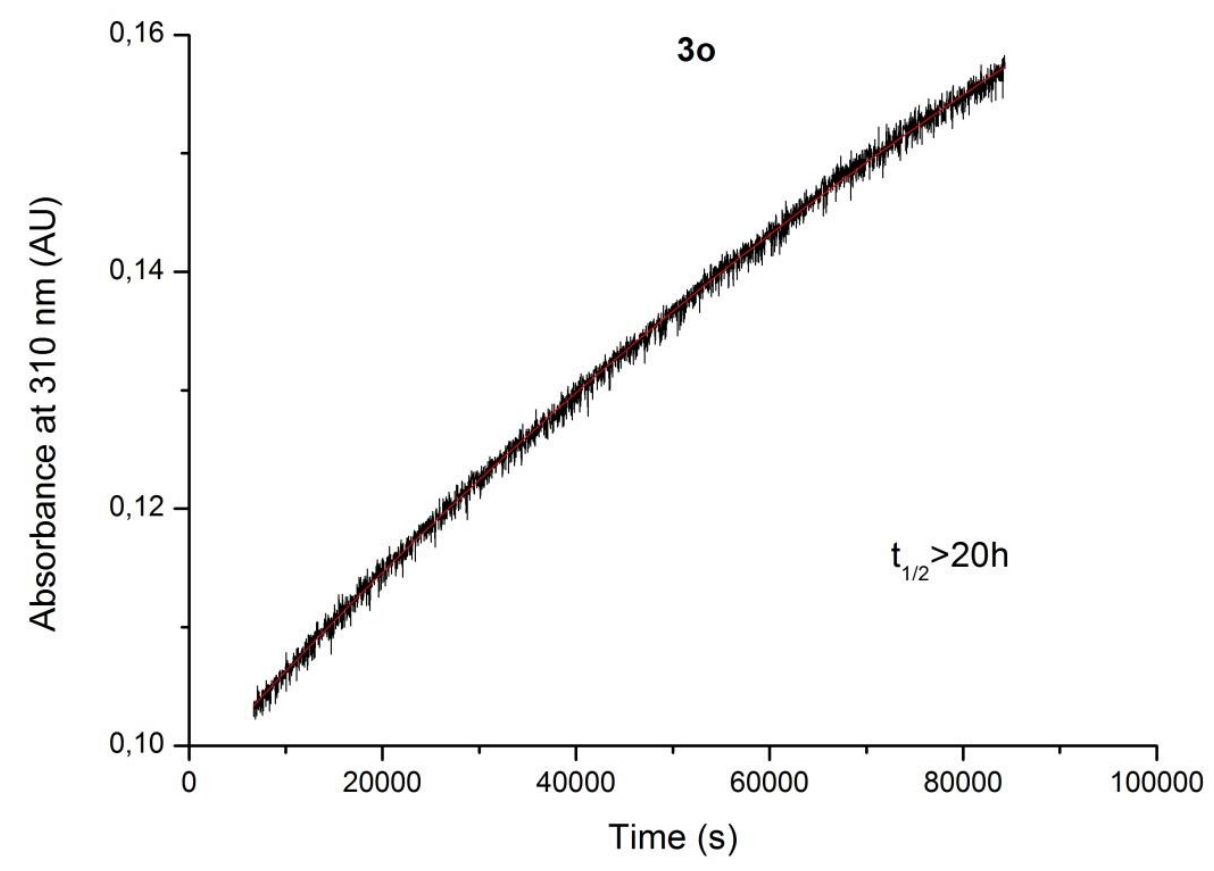

Figure S7. Determination of half-life for 30 at $37^{\circ} \mathrm{C}$ in DMSO. First, PSS was reached upon $530 \mathrm{~nm}$ irradiation, after which the absorption was measured at $\lambda_{\max }=310 \mathrm{~nm}$. Line presents the fitting with single exponential process. 


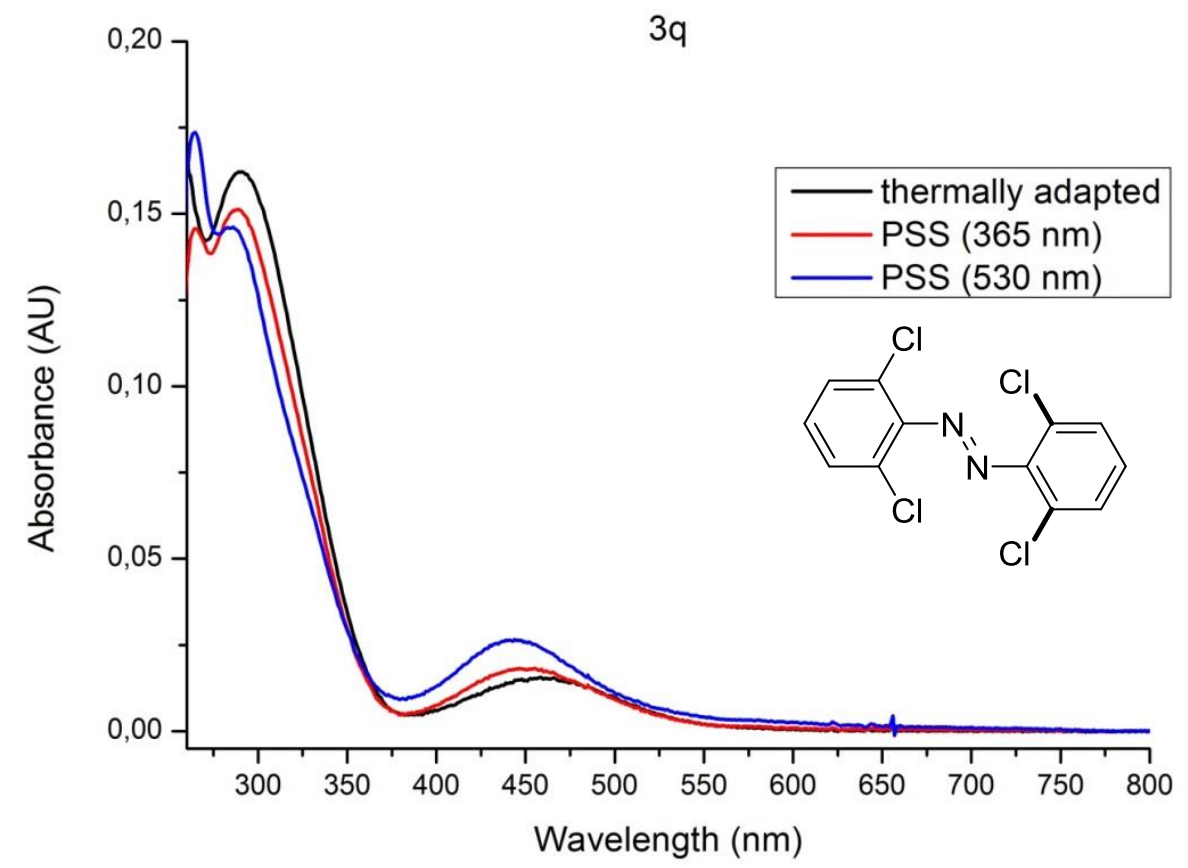

Figure S8. Absorption spectra for the photoisomerization of compound $\mathbf{3 q}\left(\mathrm{DMSO}, 37{ }^{\circ} \mathrm{C}, 20 \mu \mathrm{M}\right)$. Photostationary states after thermal adaptation, $365 \mathrm{~nm}$ irradiation and $530 \mathrm{~nm}$ irradiation were obtained.

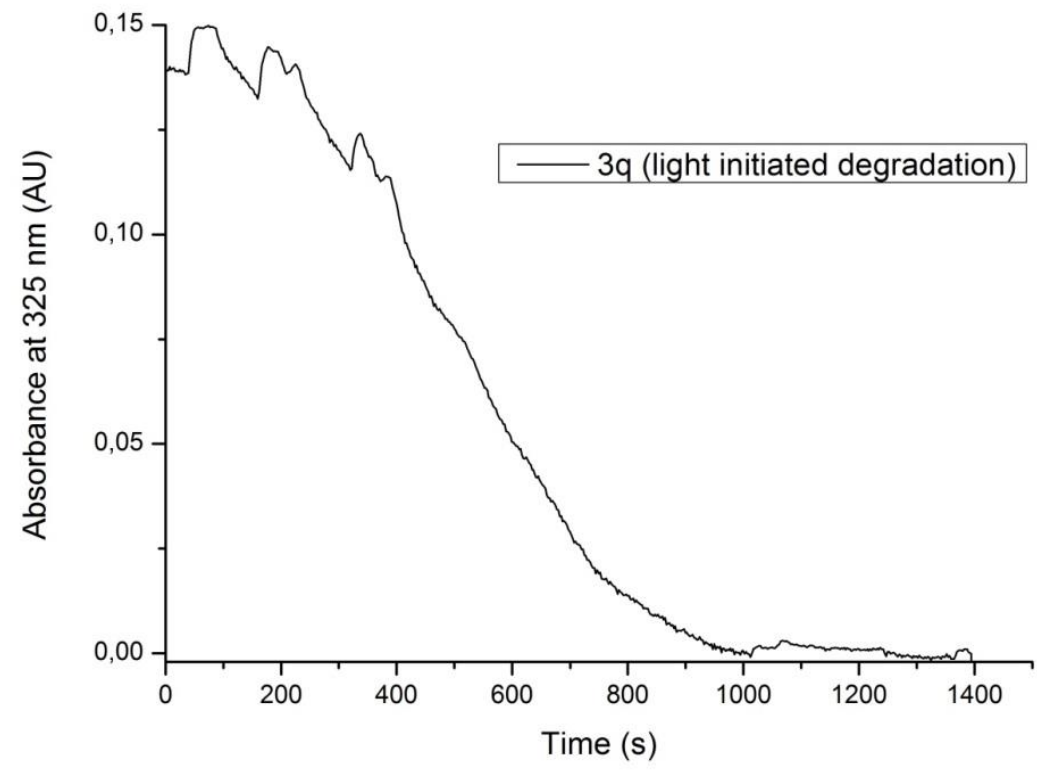

Figure S9. Reversible photosisomerization of $\mathbf{3 q}$ ( $37{ }^{\circ} \mathrm{C}$, Buffer with 1 vol\% DMSO at pH=7, $20 \mu \mathrm{M}$ ) in the presence of $10 \mathrm{mM}$ glutathione $(\mathrm{GSH})$. After two cycles of $530 \mathrm{~nm} /$ white light irradiation, degradation was initiated which led to a full reduction of the absorbance within 20 minutes. This was irreversible and could not be prevented by white light irradiation, $530 \mathrm{~nm}$ irradiation or no light exposure. 


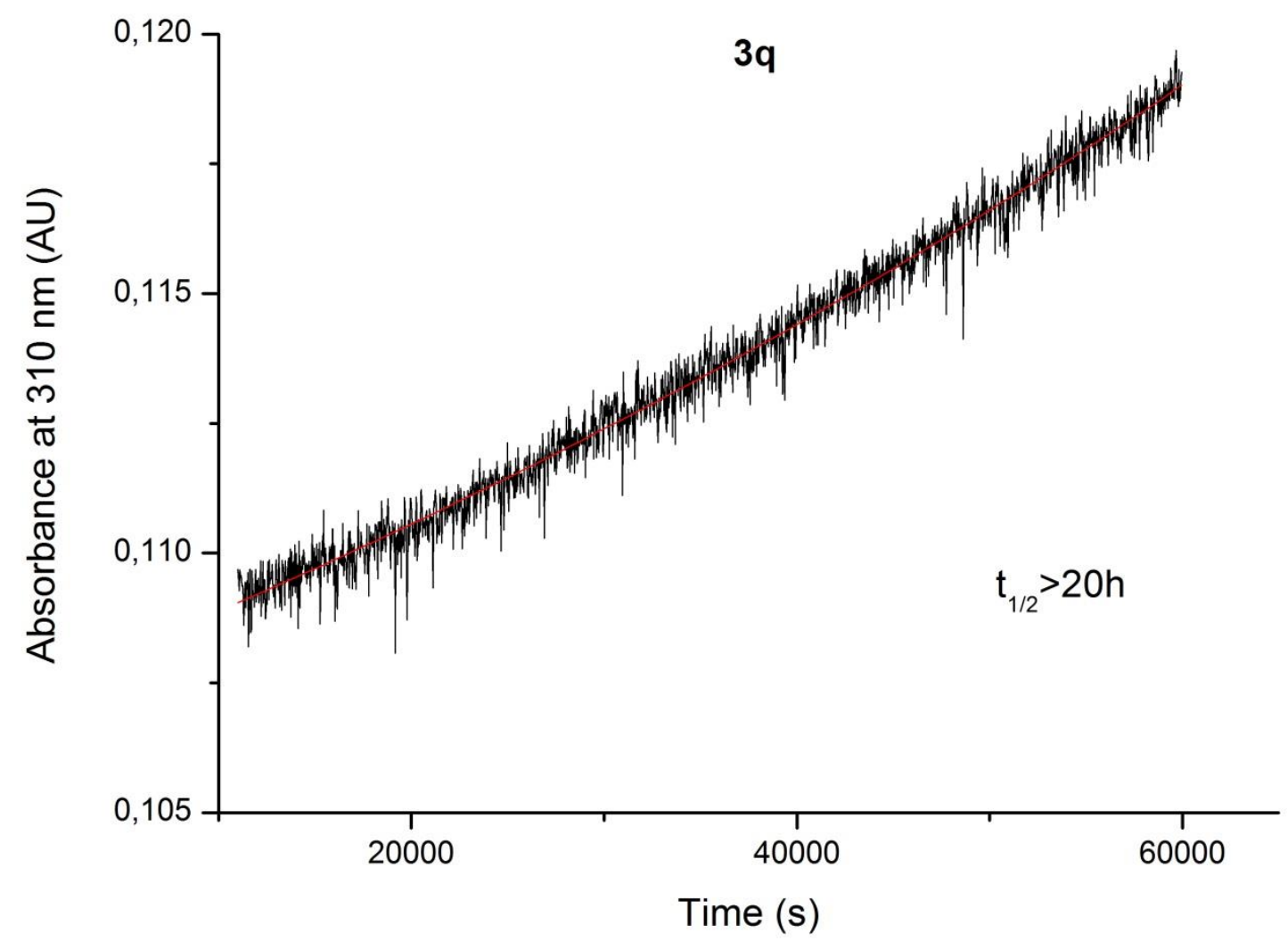

Figure S10. Determination of half-life for $\mathbf{3 q}$ at $37{ }^{\circ} \mathrm{C}$ in DMSO. First, PSS was reached upon $530 \mathrm{~nm}$ irradiation, after which the absorption was measured at $\lambda_{\max }=310 \mathrm{~nm}$. Line presents the fitting with single exponential process. 


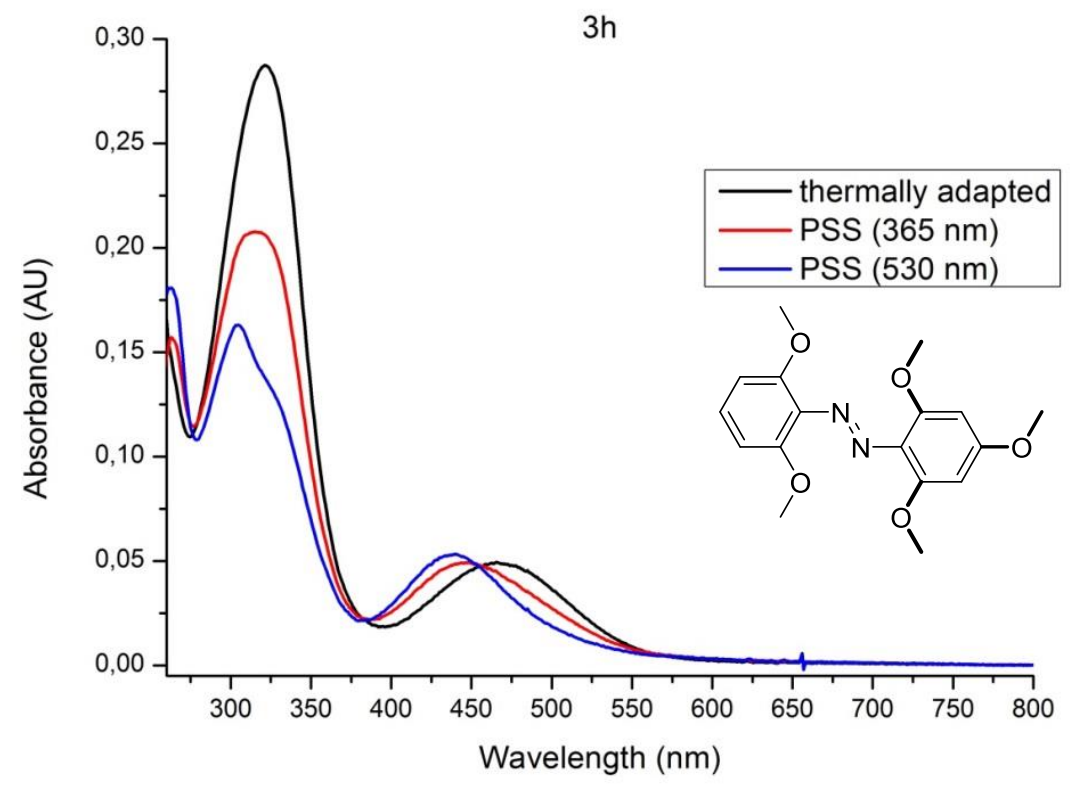

Figure S11. Absorption spectra for the photoisomerization of compound $\mathbf{3 h}\left(\mathrm{DMSO}, 37{ }^{\circ} \mathrm{C}, 20 \mu \mathrm{M}\right)$. Photostationary states after thermal adaptation, $365 \mathrm{~nm}$ irradiation and $530 \mathrm{~nm}$ irradiation were obtained.

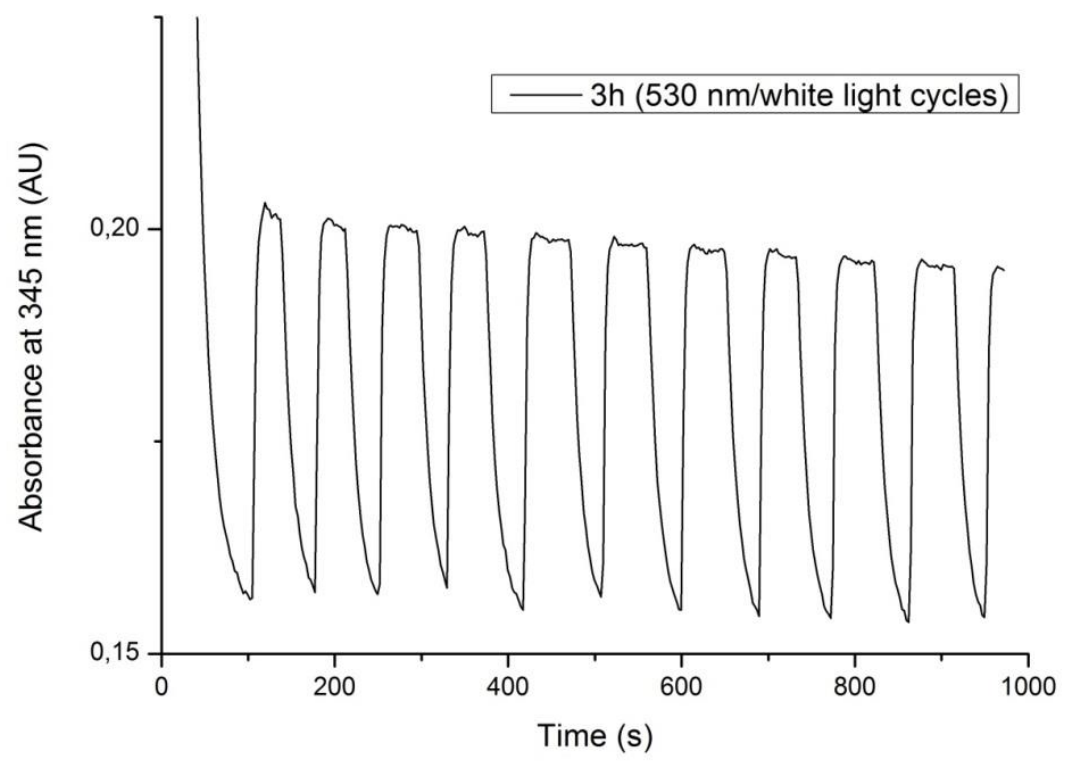

Figure S12. Reversible photosisomerization of $\mathbf{3 h}$ ( $37^{\circ} \mathrm{C}$, Buffer with 1 vol\% DMSO at $\mathrm{pH}=7,20 \mu \mathrm{M}$ ) in the presence of $10 \mathrm{mM}$ glutathione $(\mathrm{GSH})$. Ten cycles of $530 \mathrm{~nm} /$ white light irradiation could be performed without the observation of significant fatigue or reduction. 


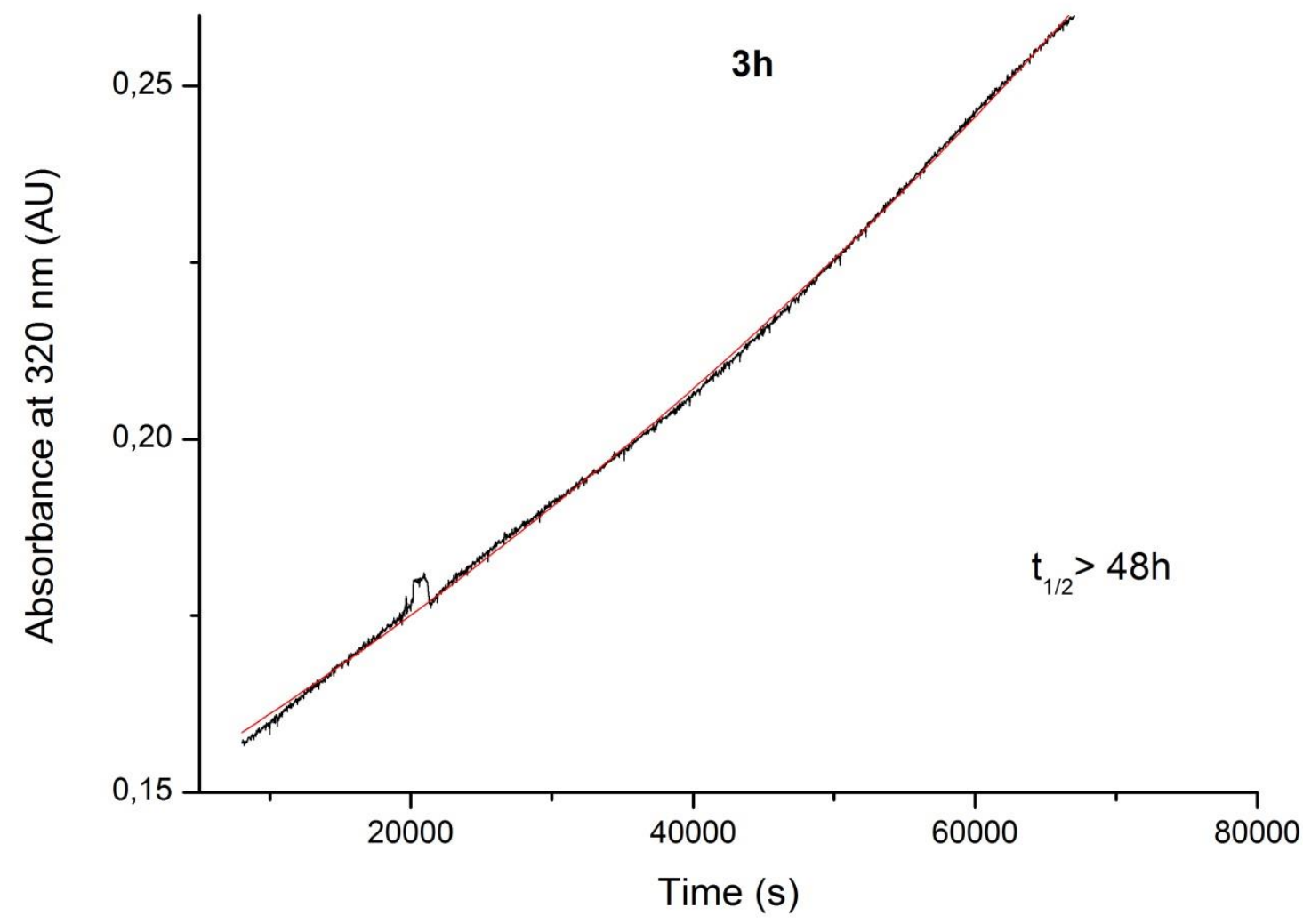

Figure S13. Determination of half-life for $3 \mathbf{h}$ at $37{ }^{\circ} \mathrm{C}$ in DMSO. First, PSS was reached upon $530 \mathrm{~nm}$ irradiation, after which the absorption was measured at $\lambda_{\max }=310 \mathrm{~nm}$. Line presents the fitting with single exponential process. 


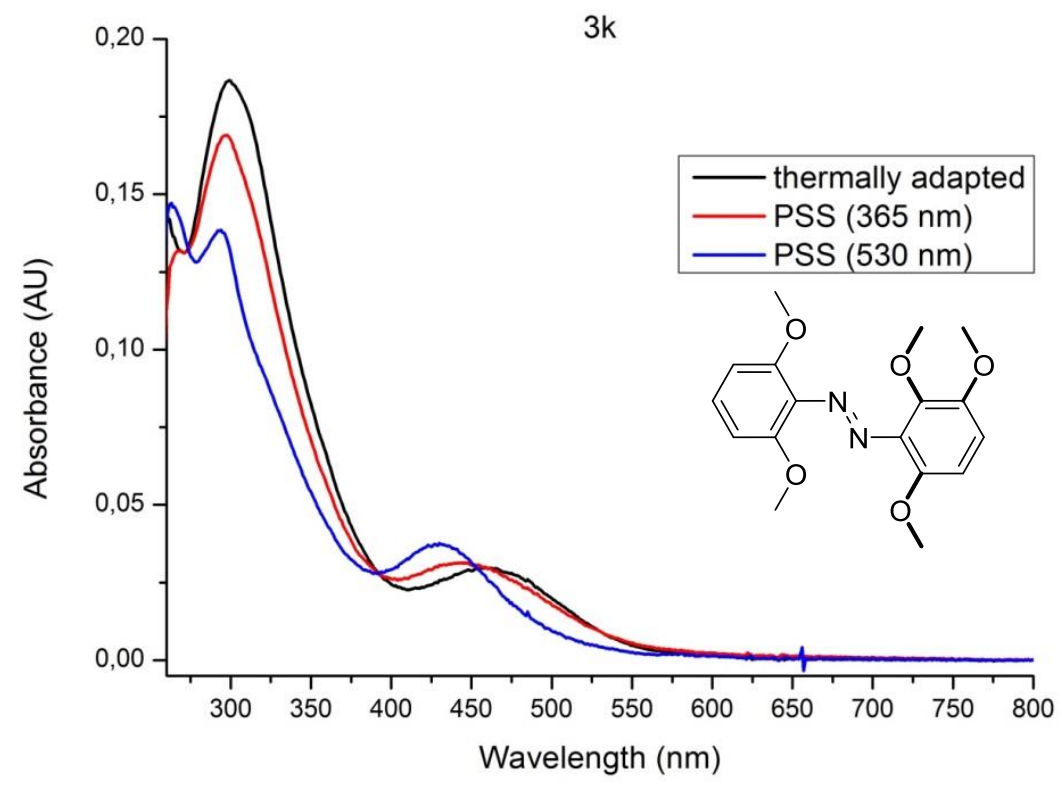

Figure S14. Absorption spectra for the photoisomerization of compound 3k (DMSO, $\left.37{ }^{\circ} \mathrm{C}, 20 \mu \mathrm{M}\right)$. Photostationary states after thermal adaptation, $365 \mathrm{~nm}$ irradiation and $530 \mathrm{~nm}$ irradiation were obtained.

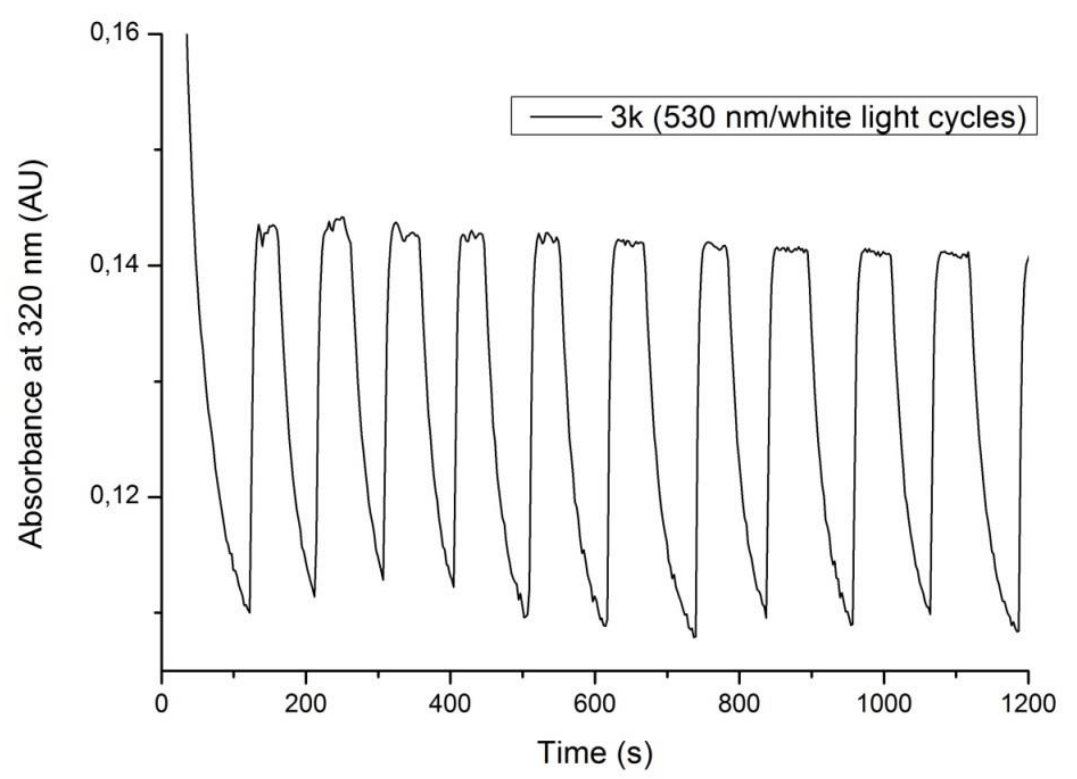

Figure S15. Reversible photosisomerization of 3k ( $37^{\circ} \mathrm{C}$, Buffer with 1 vol\% DMSO at $\mathrm{pH}=7,20 \mu \mathrm{M}$ ) in the presence of $10 \mathrm{mM}$ glutathione $(\mathrm{GSH})$. Ten cycles of $530 \mathrm{~nm} /$ white light irradiation could be performed without the observation of significant fatigue or reduction. 


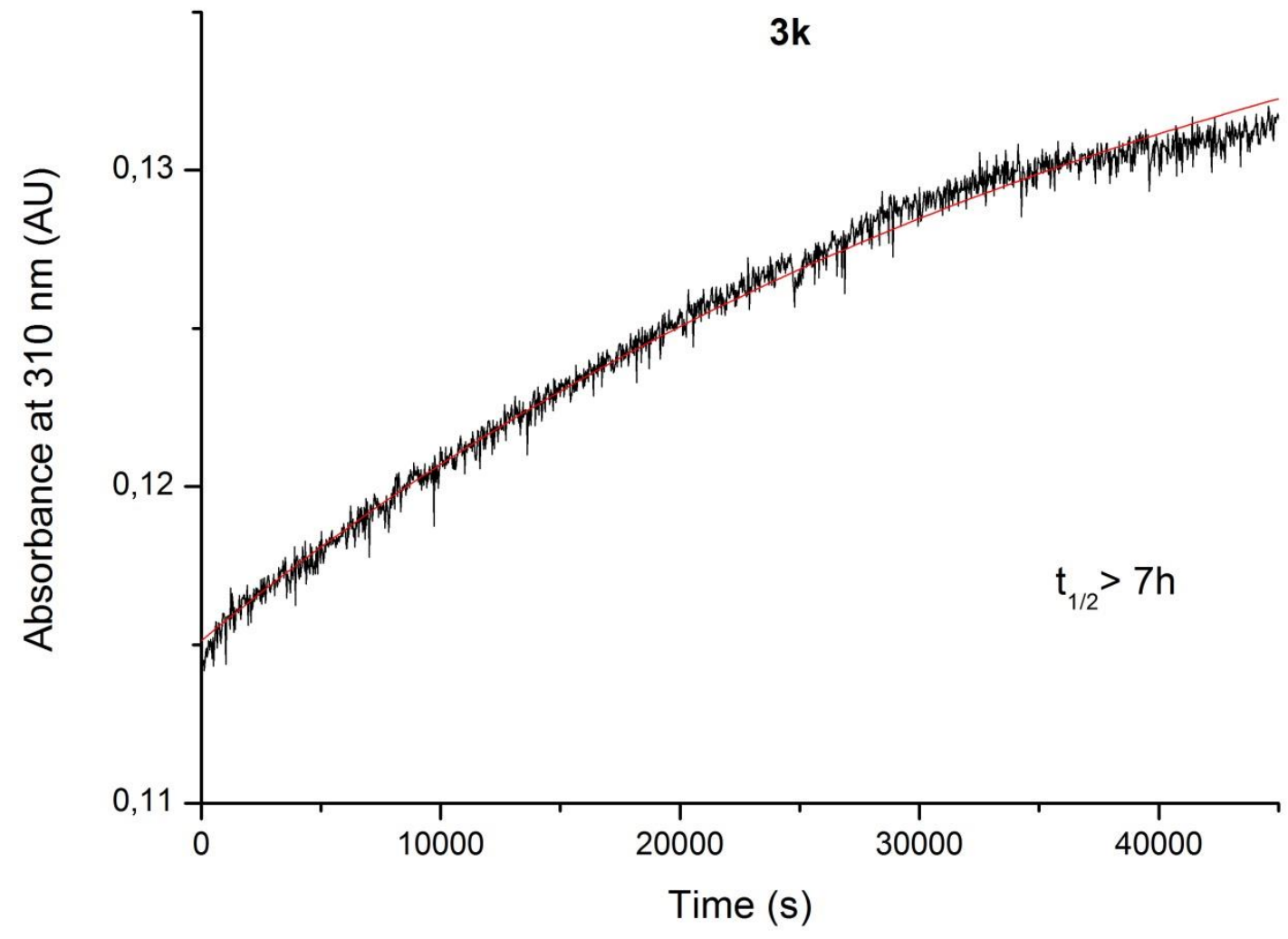

Figure S16. Determination of half-life for 3k at $37{ }^{\circ} \mathrm{C}$ in DMSO. First, PSS was reached upon $530 \mathrm{~nm}$ irradiation, after which the absorption was measured at $\lambda_{\max }=310 \mathrm{~nm}$. Line presents the fitting with single exponential process. 


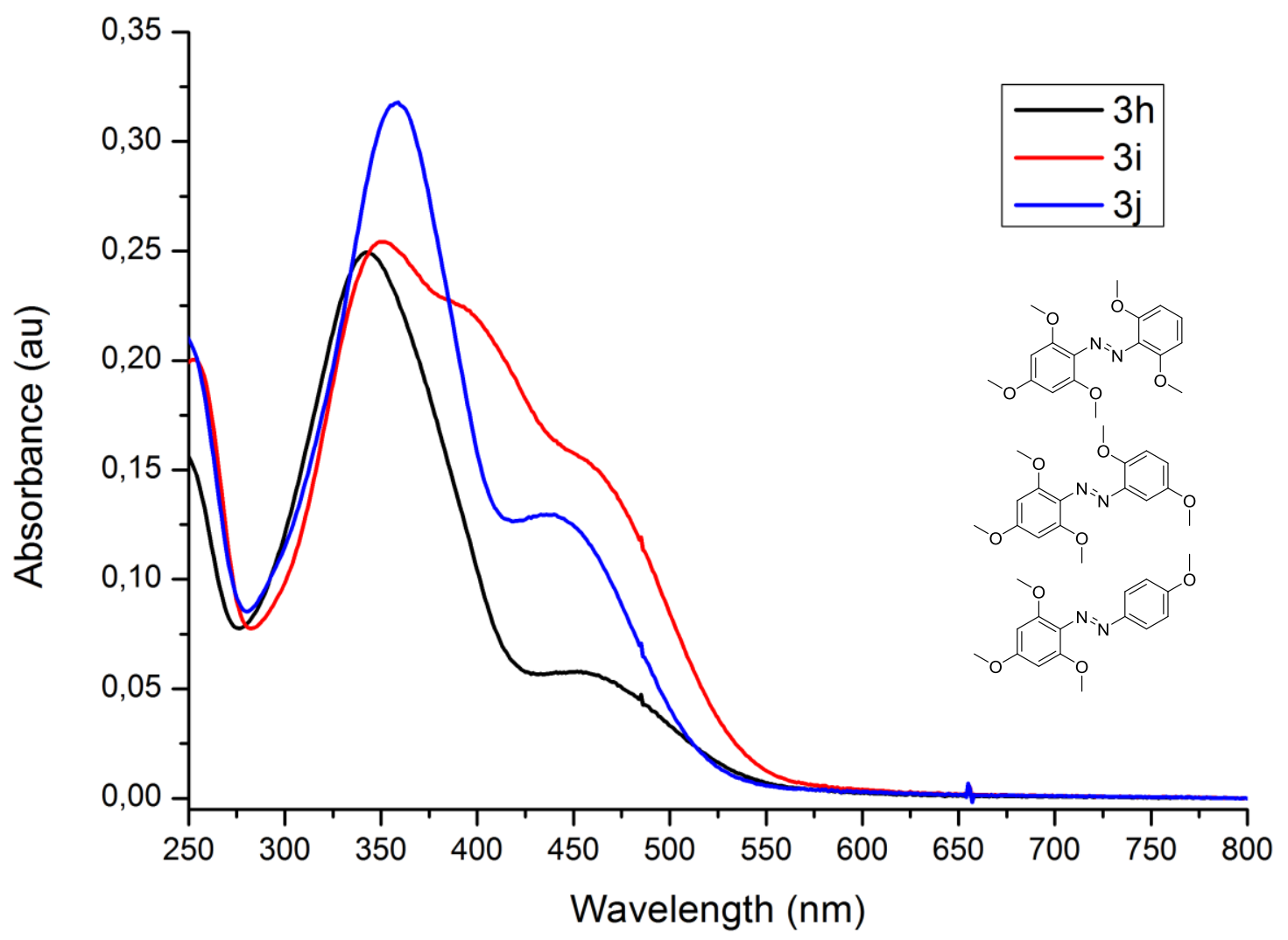

Figure S17. Absorption spectra of compound $\mathbf{3 h}-\mathbf{j}(20 \mu \mathrm{M}$ in buffer with 1 vol\% DMSO at $\mathrm{pH}=7)$ showing the distinct differences in absorption spectrum caused by a changing substitution pattern $(2,6-$ dimethoxy to 2,5-dimethoxy to 4-methoxy substituents). 


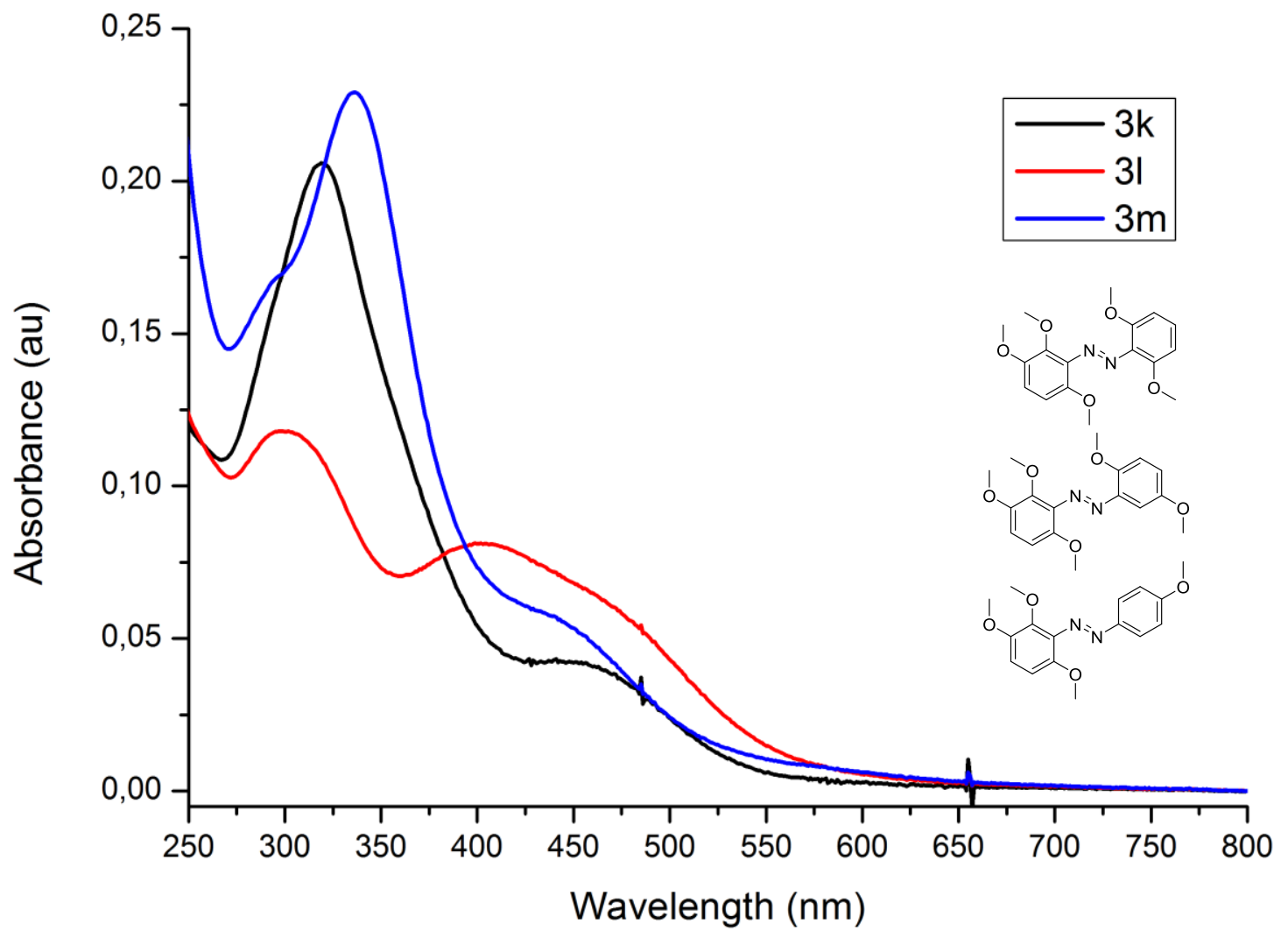

Figure S18. Absorption spectra of compound 3k-m $(20 \mu \mathrm{M}$ in buffer with 1 vol\% DMSO at $\mathrm{pH}=7)$ showing the distinct differences in absorption spectrum caused by a changing substitution pattern $(2,6-$ dimethoxy to 2,5-dimethoxy to 4-methoxy substituents). 


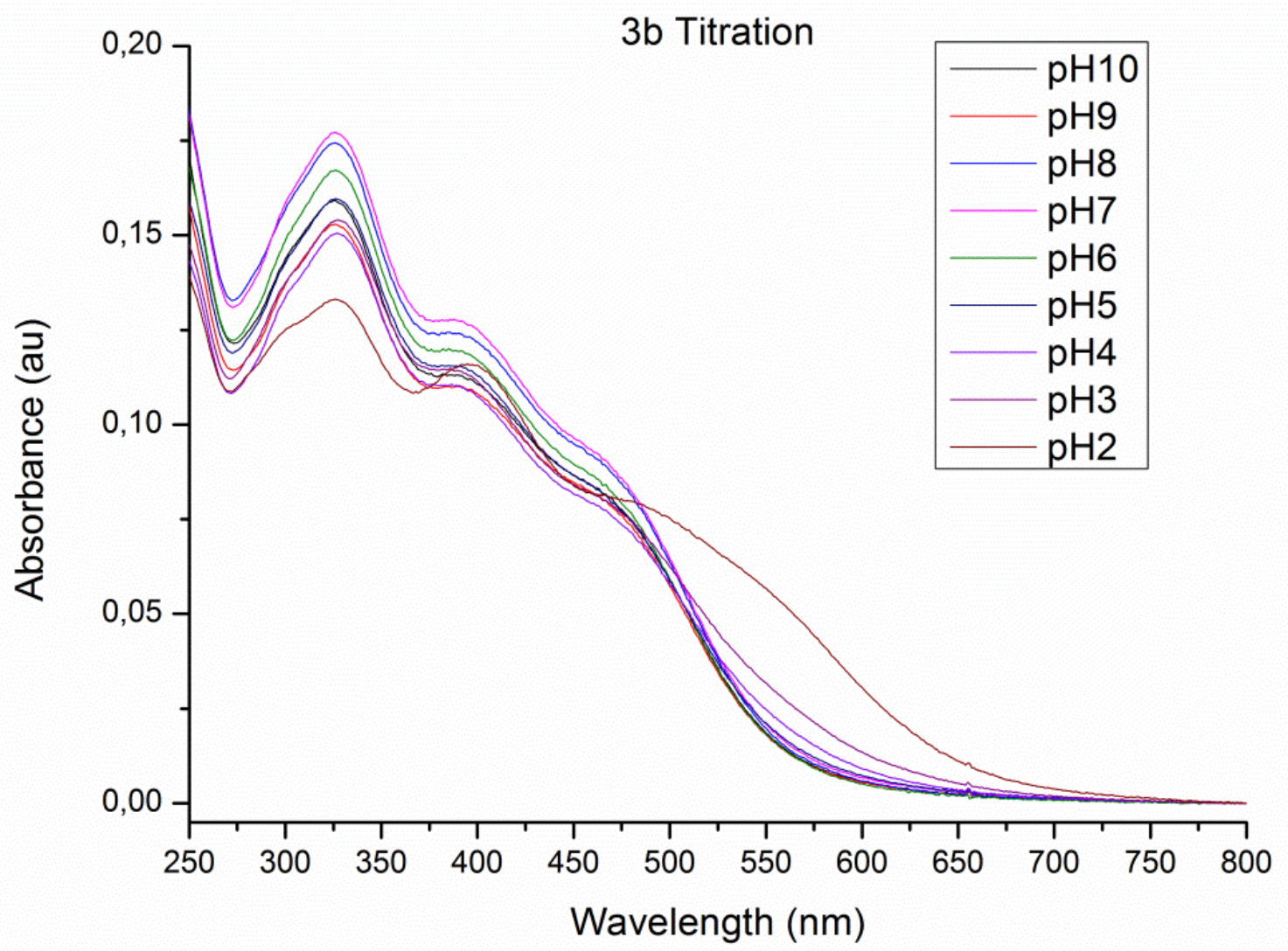

Figure S19. Titration curve of compound 3b $(20 \mu \mathrm{M}$ in buffer with 1 vol\% DMSO) showing the different absorption spectra at different $\mathrm{pH}$. 


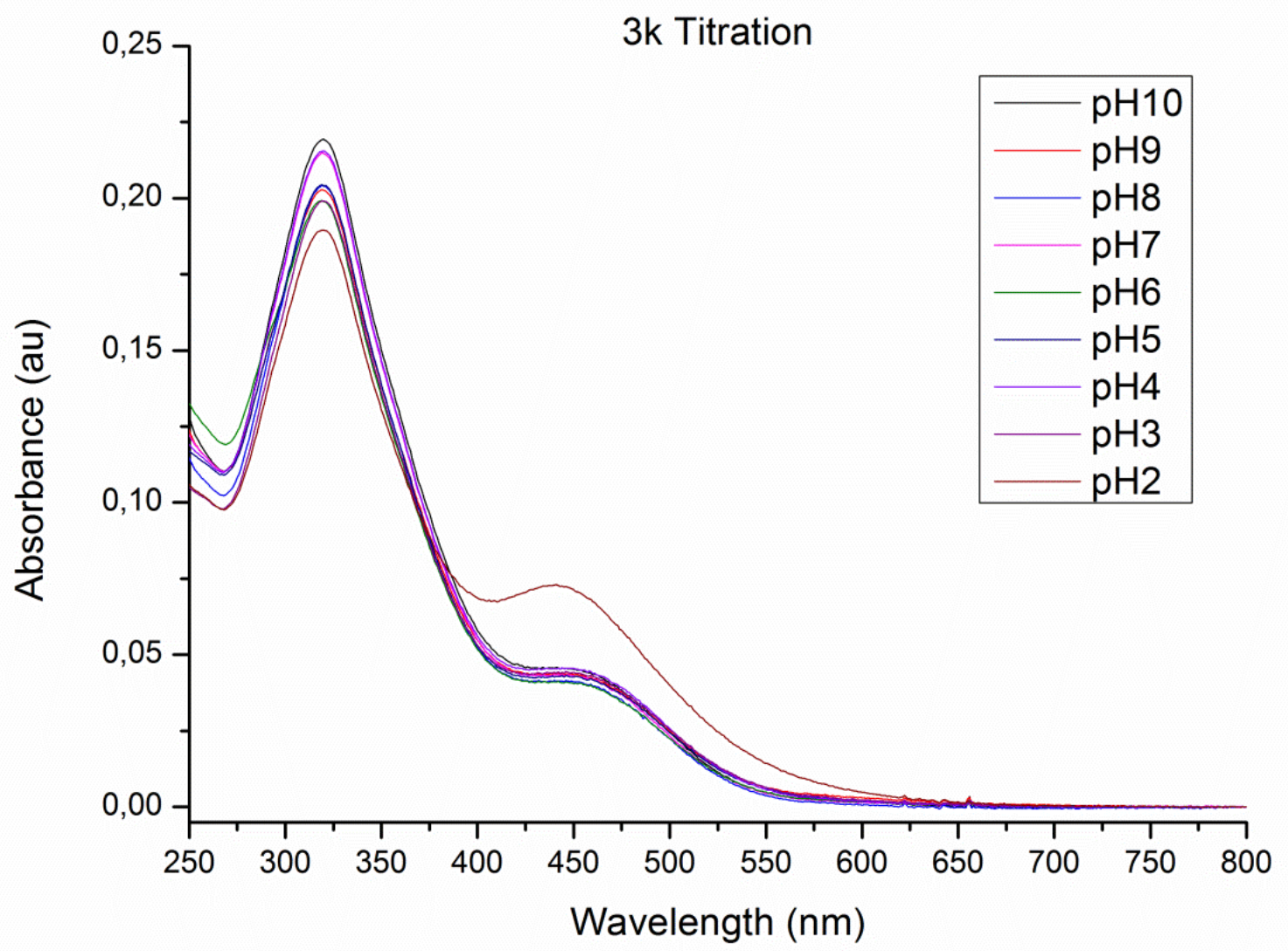

Figure S20.Titration curve of compound 3k $(20 \mu \mathrm{M}$ in buffer with 1 vol\% DMSO) showing the different absorption spectra at different $\mathrm{pH}$. 
Table S3. Photostationary states (PSS, DMSO- $\mathrm{d}_{6},{ }^{1} \mathrm{H}-\mathrm{NMR}$ ) at different wavelengths of irradiation.

\begin{tabular}{|c|c|c|c|}
\hline Compound & $\begin{array}{l}\text { Trans:cis (thermally } \\
\text { adapted) }\end{array}$ & $\begin{array}{l}\text { Trans:cis (365 } \mathrm{nm} \\
\text { irradiated) }\end{array}$ & $\begin{array}{l}\text { Trans:cis (530 } \mathrm{nm} \\
\text { irradiated) }\end{array}$ \\
\hline 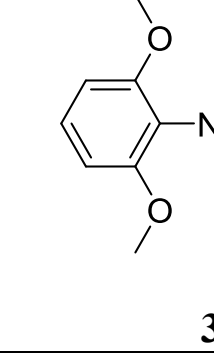 & $97: 3$ & $67: 33$ & $17: 83$ \\
\hline & . & $75: 25$ & 8:92 \\
\hline & $>97: 3$ & $65: 35$ & 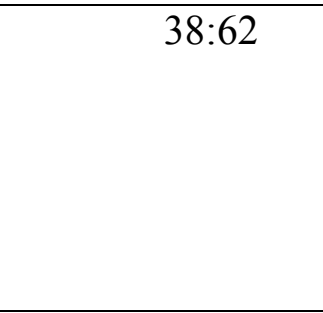 \\
\hline 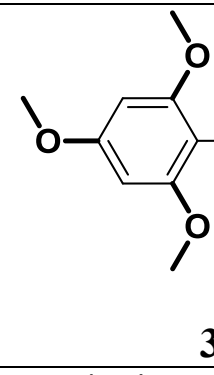 & $>97: 3$ & $54: 46$ & . \\
\hline 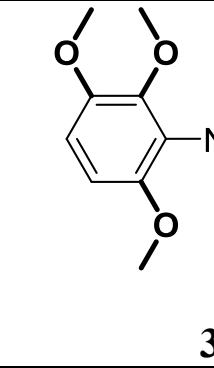 & $89: 11$ & 61:39 & $20: 80$ \\
\hline
\end{tabular}


3a (thermally adapted):

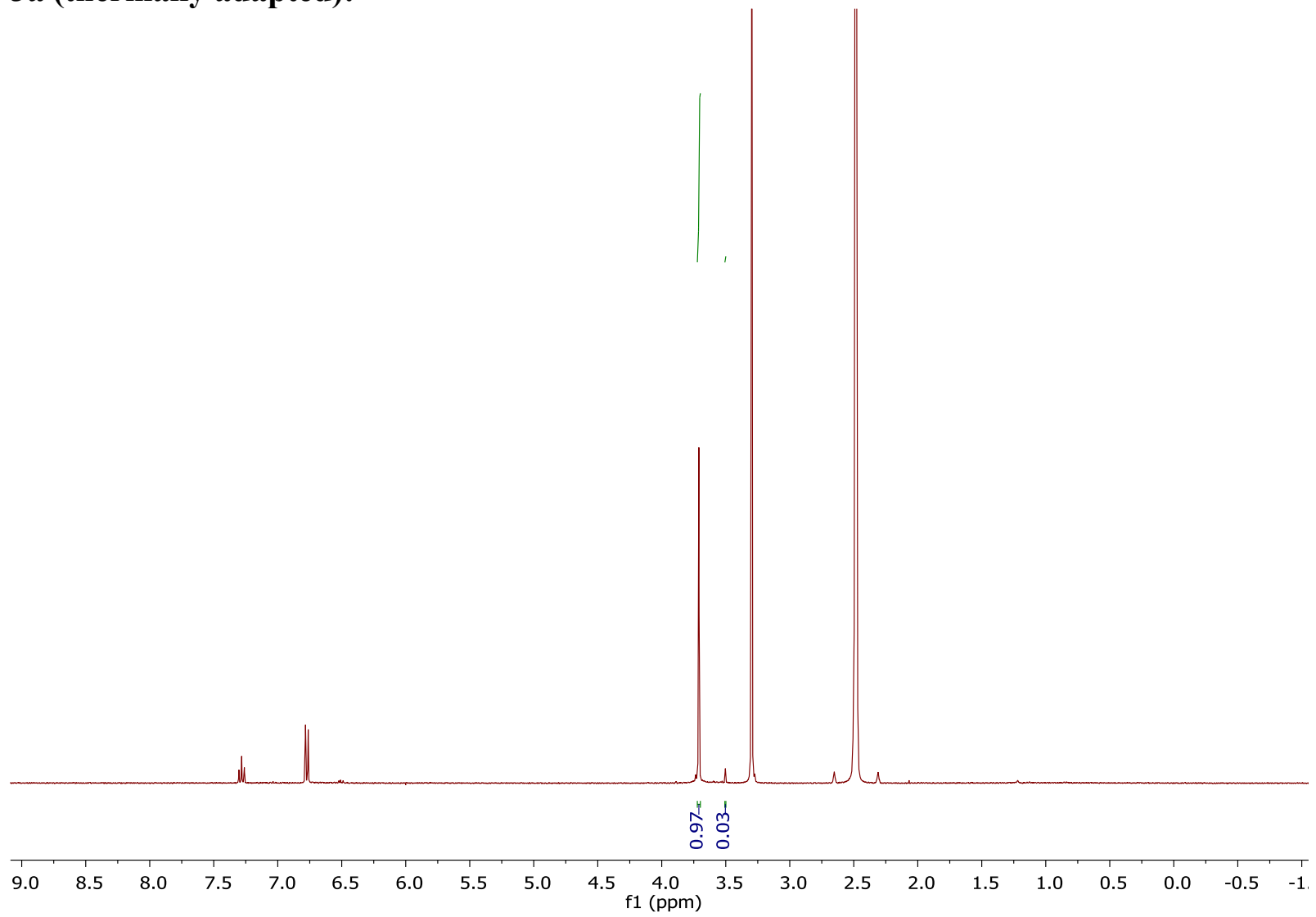

3a (PSS $365 \mathrm{~nm}$ irradiation):

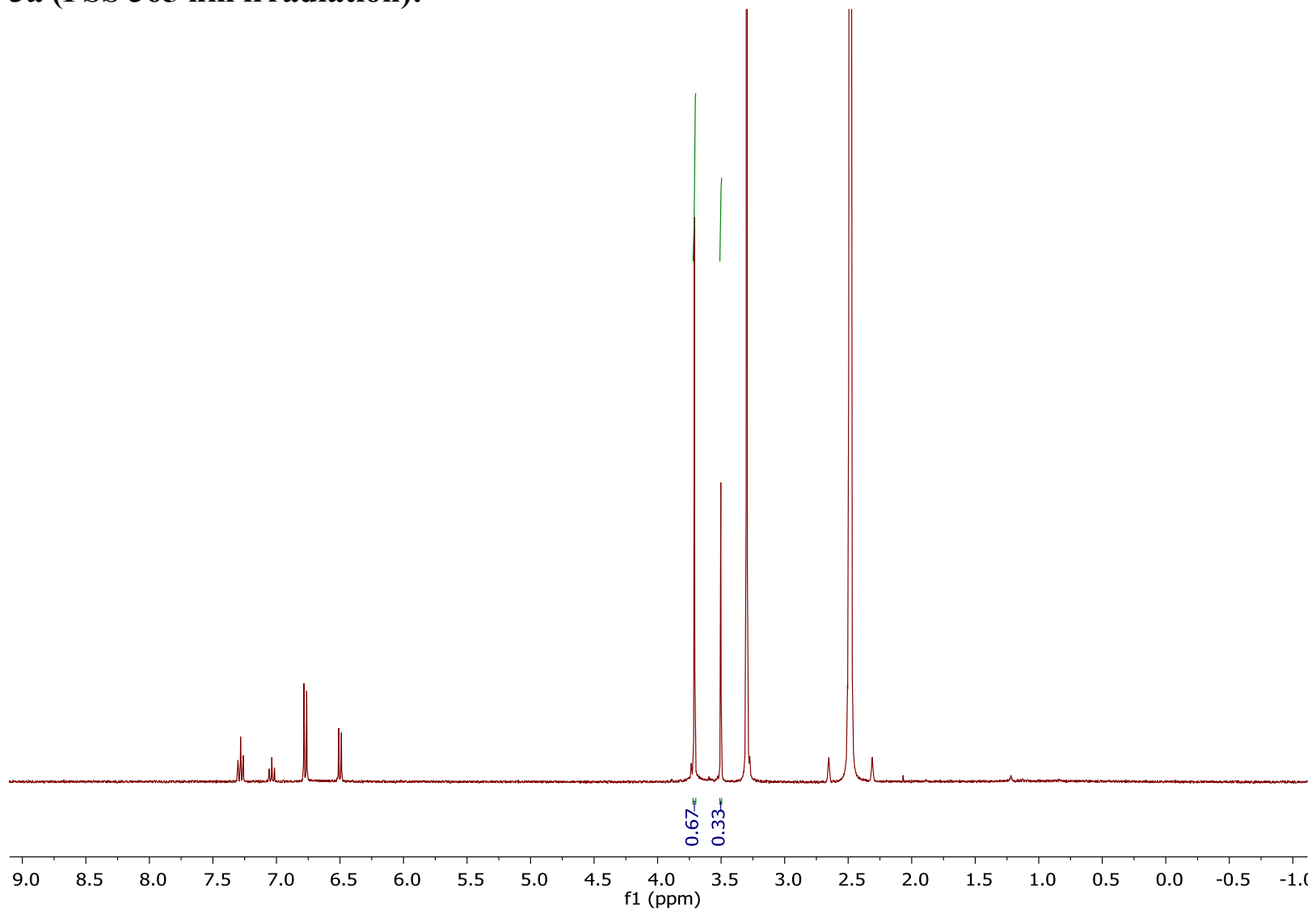


3a (PSS $530 \mathrm{~nm}$ irradiation):

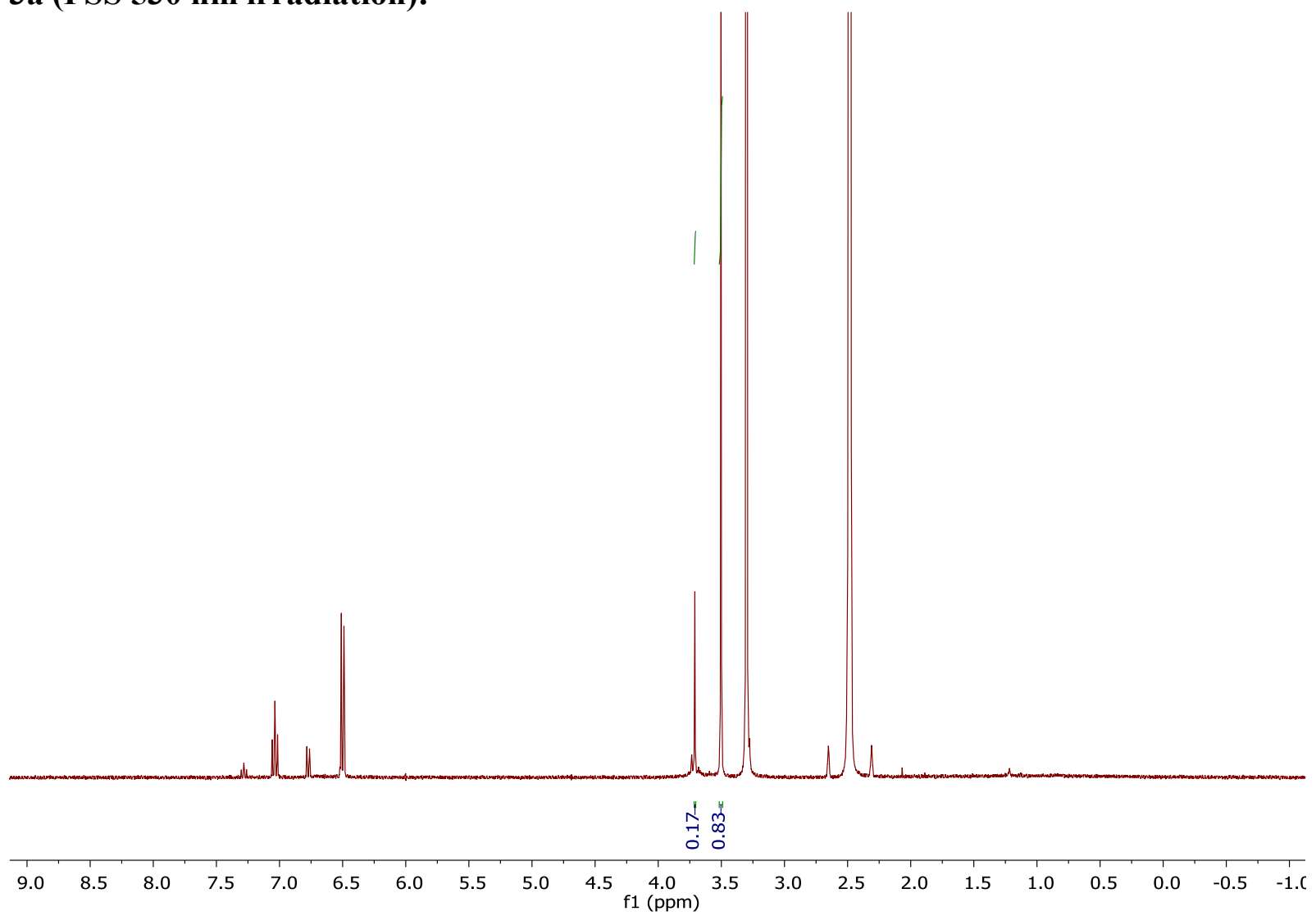

30 (thermally adapted):

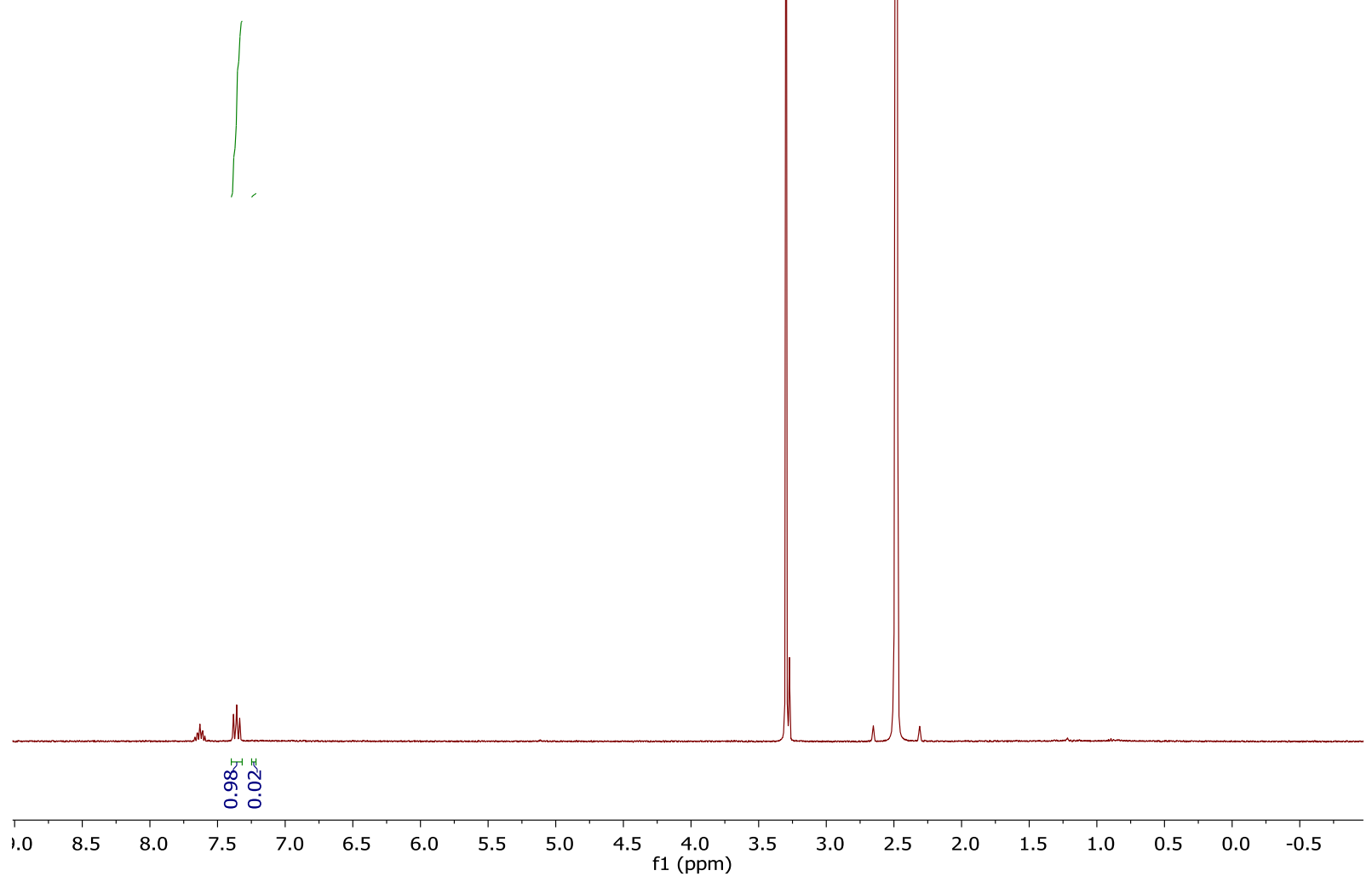


30 (PSS 365 nm irradiated):

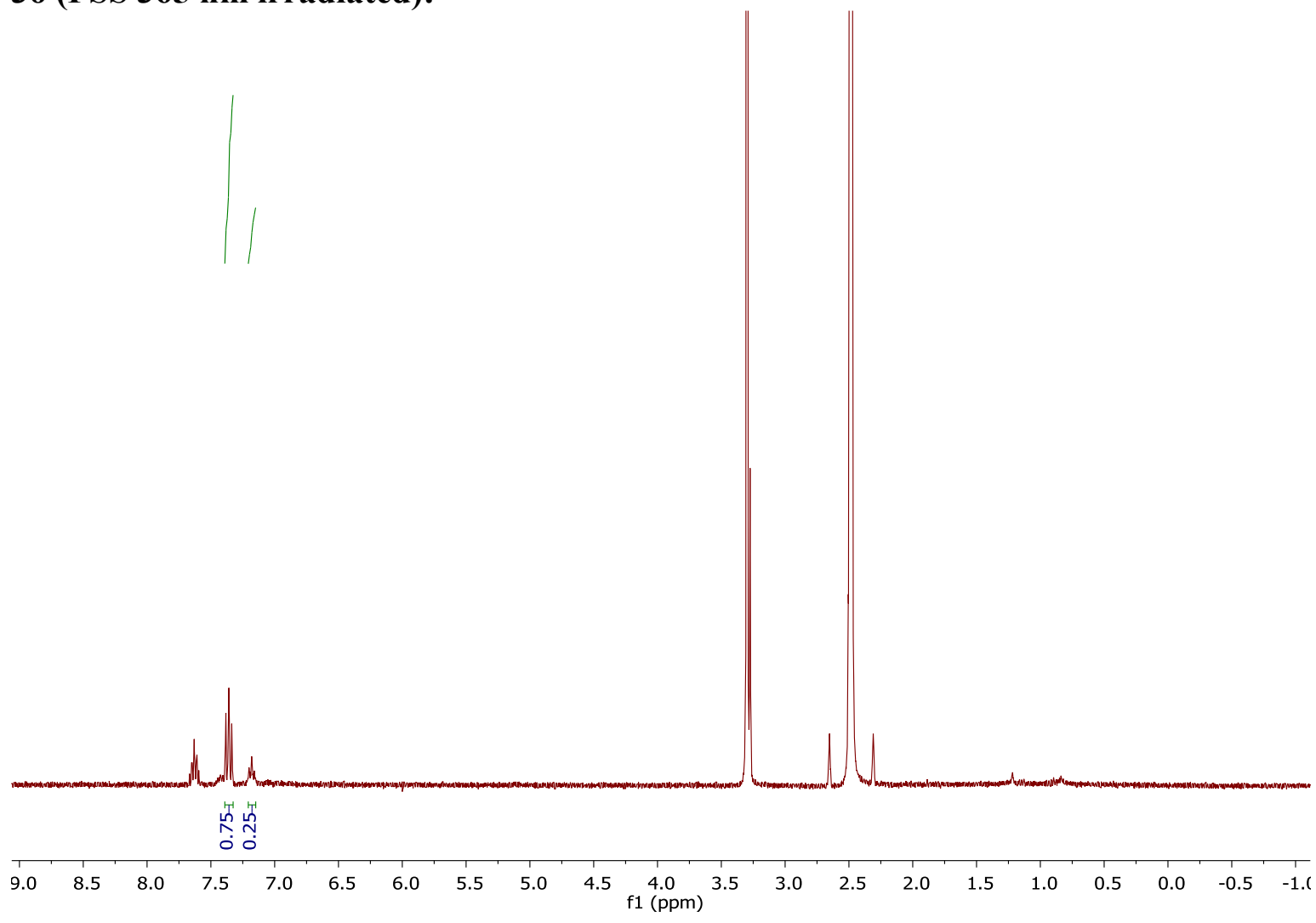

30 (PSS $530 \mathrm{~nm}$ irradiated):

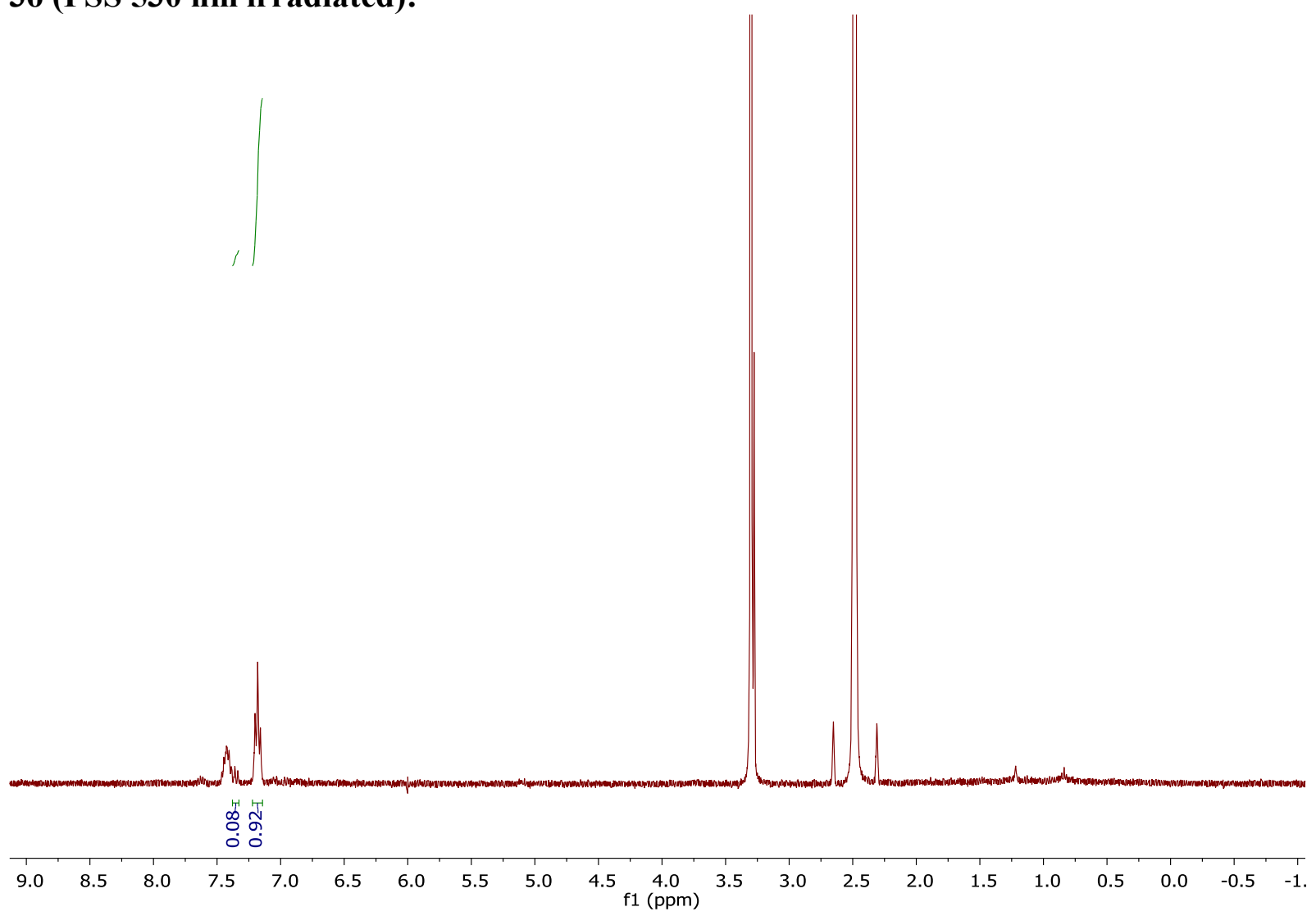


3q (thermally adapted):

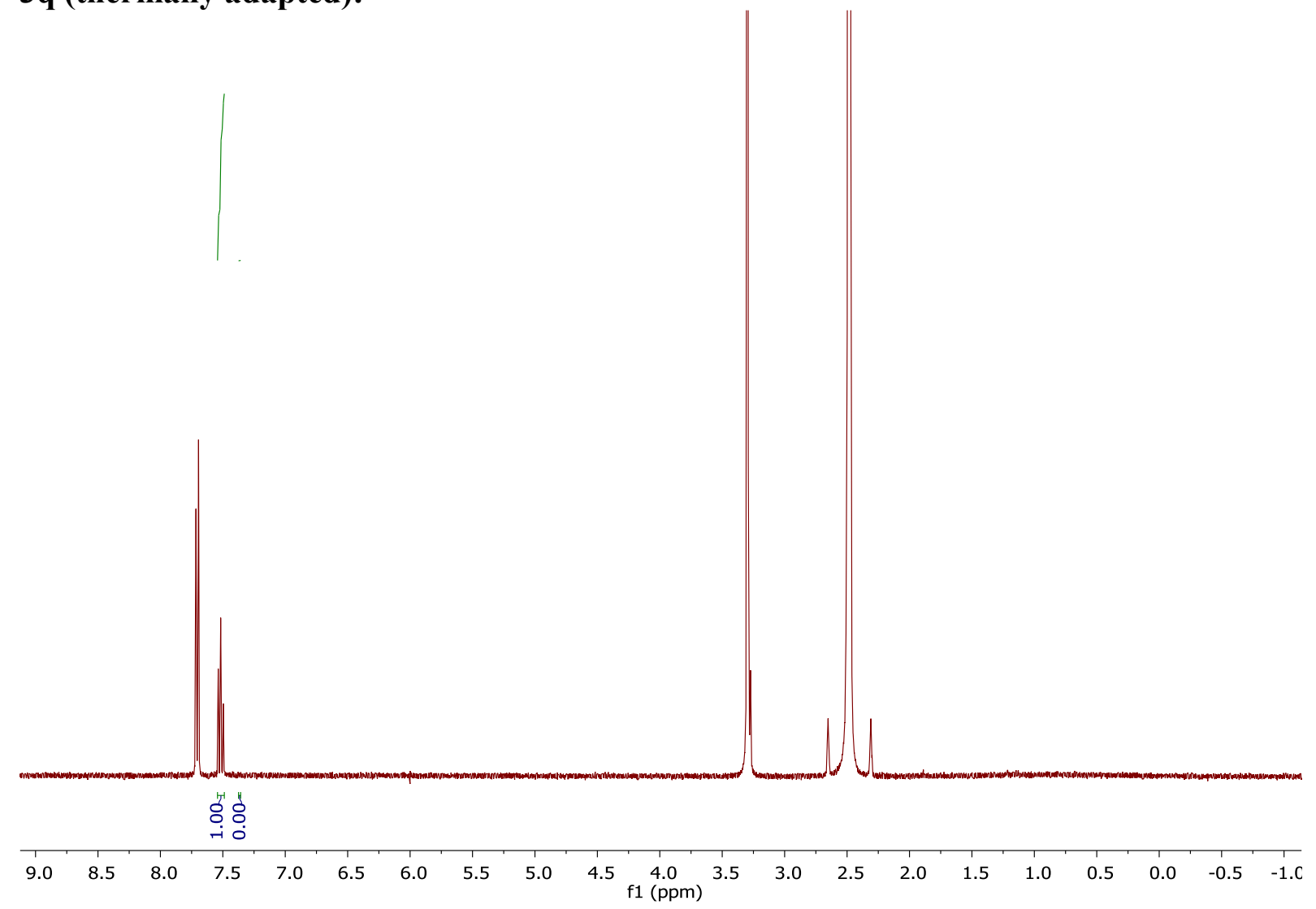

3q (PSS $365 \mathrm{~nm}$ irradiation):

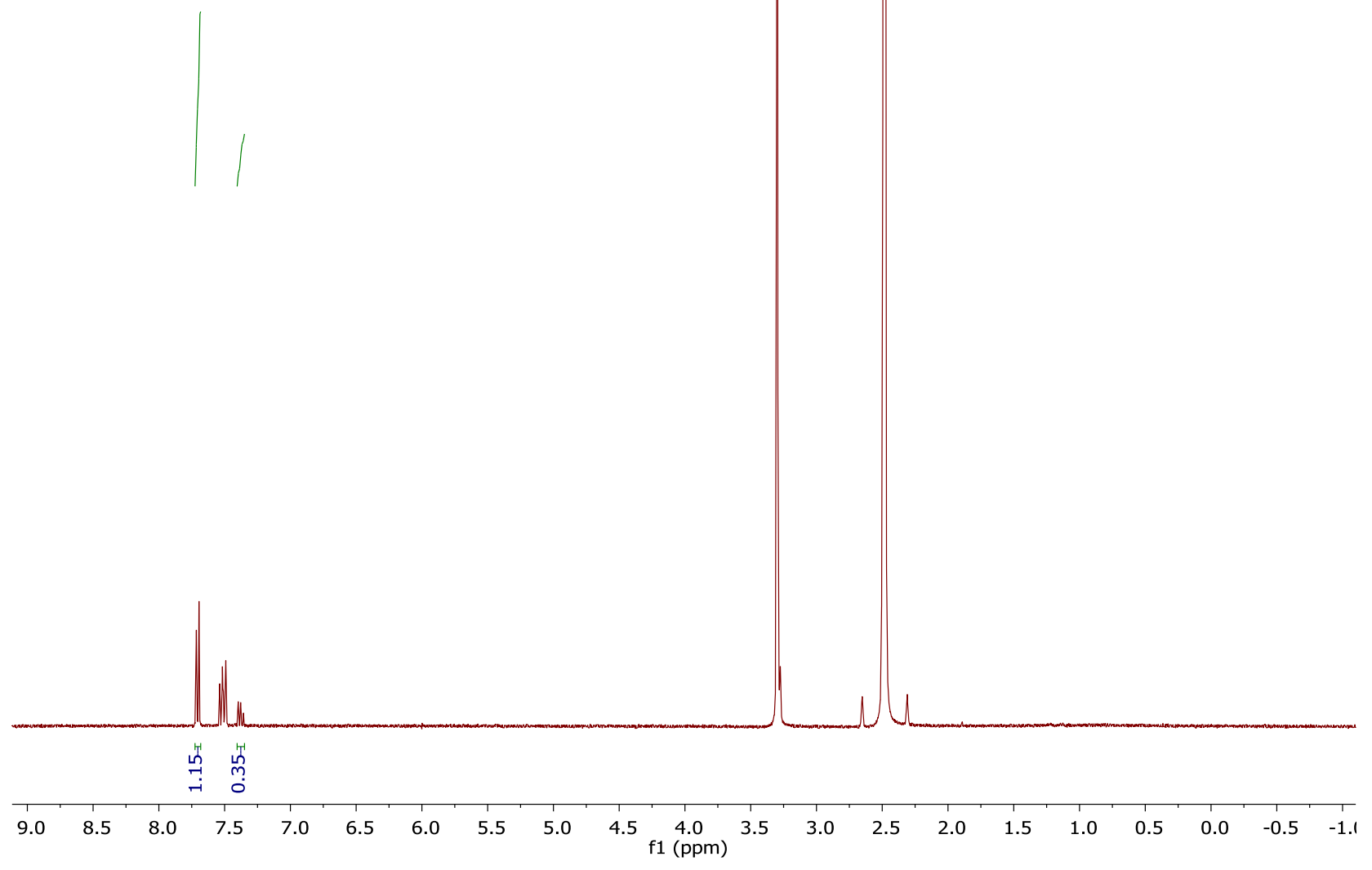


$3 q(530 \mathrm{~nm}$ irradiation $):$

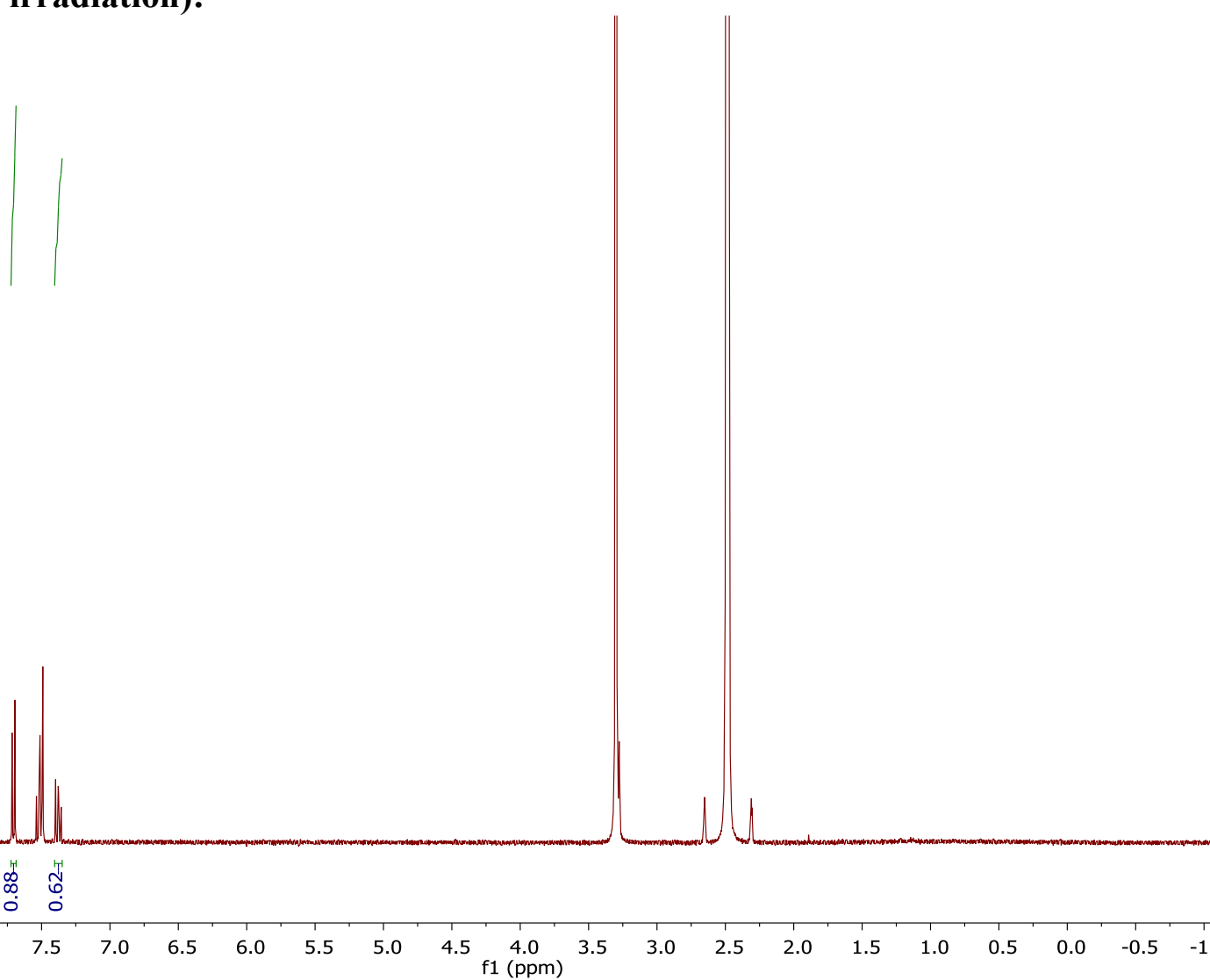

3h (thermally adapted):

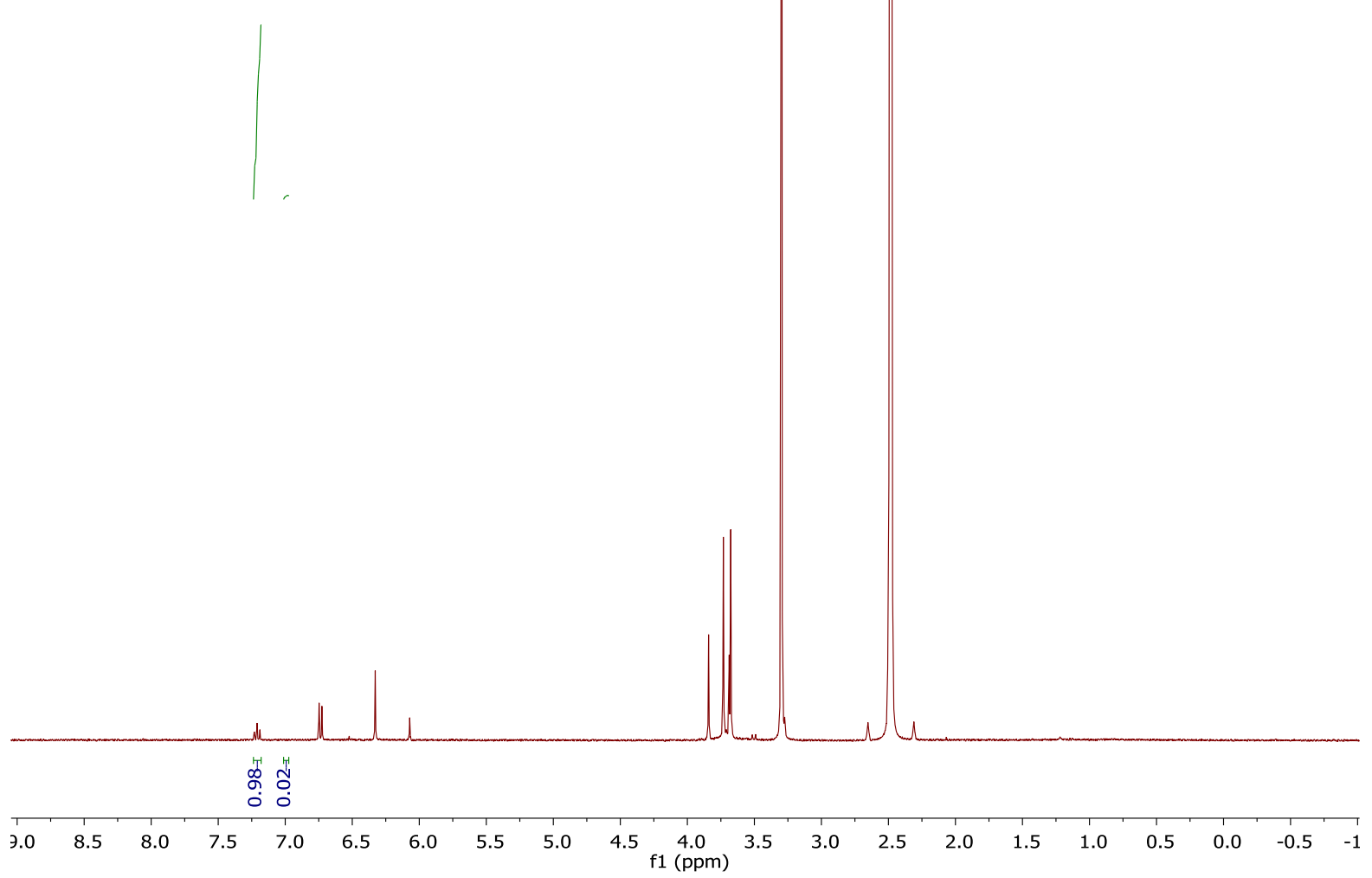


3h (PSS $365 \mathrm{~nm}$ irradiation):

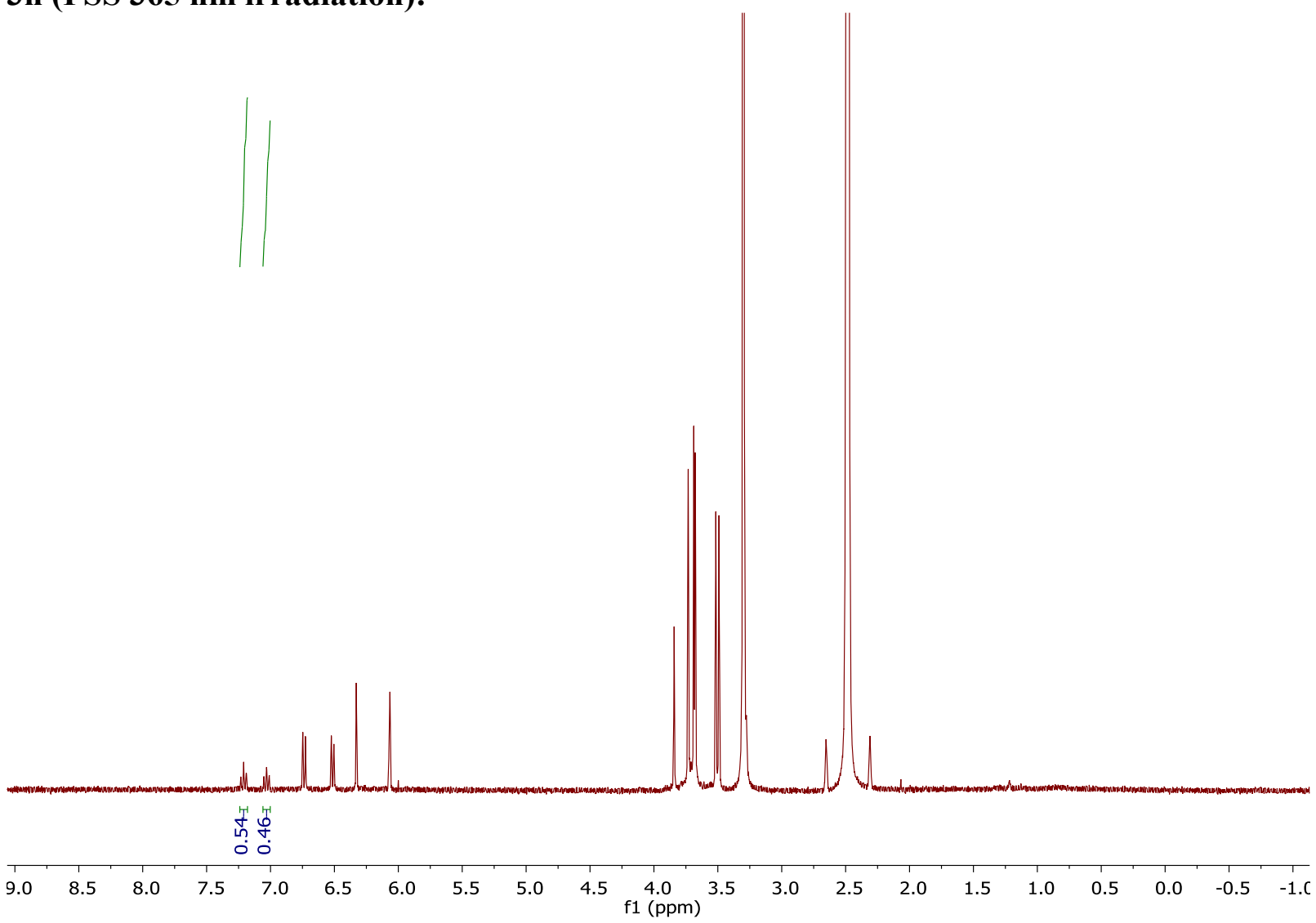

3h (PSS $530 \mathrm{~nm}$ irradiation):

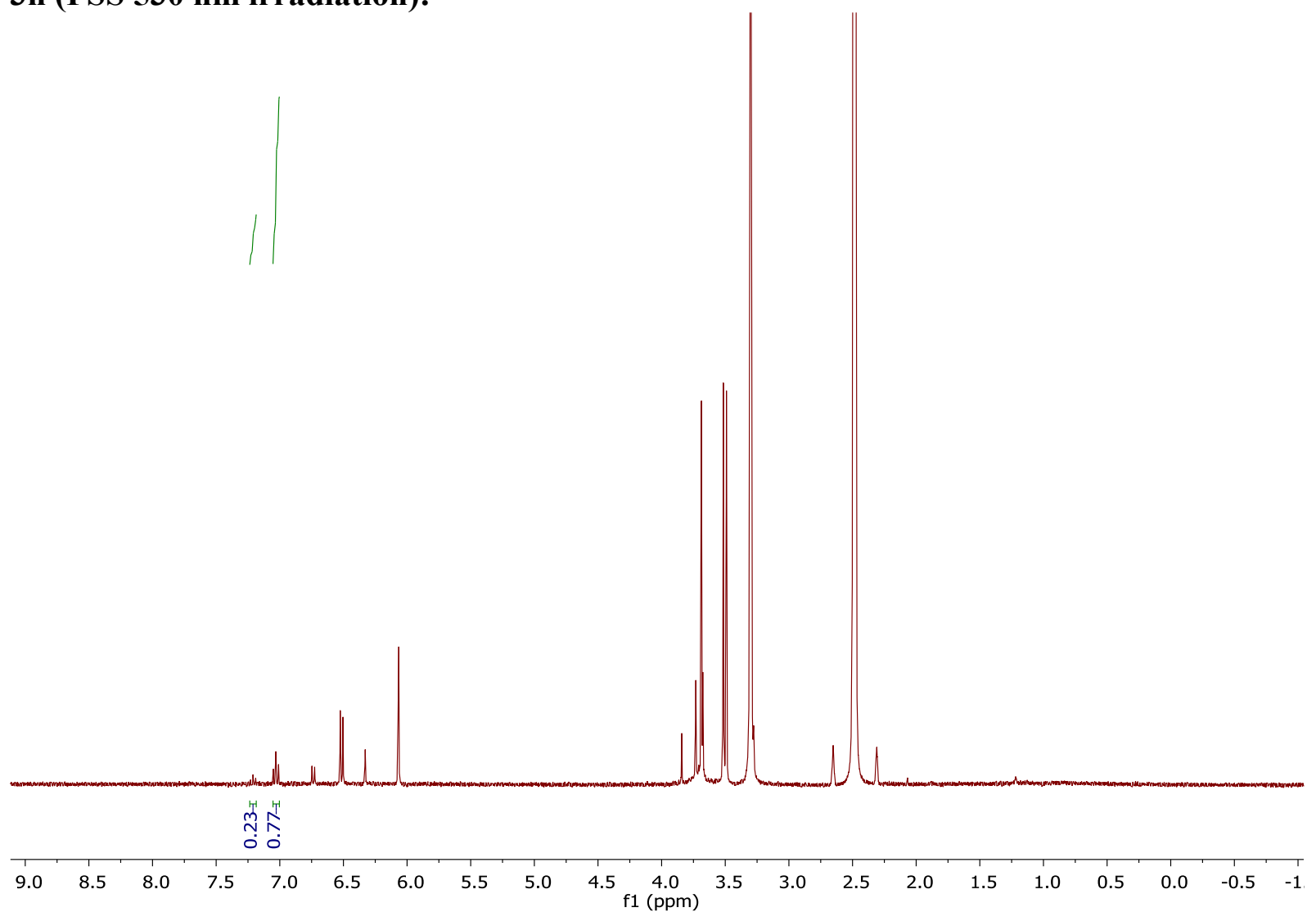


3k (thermally adapted):

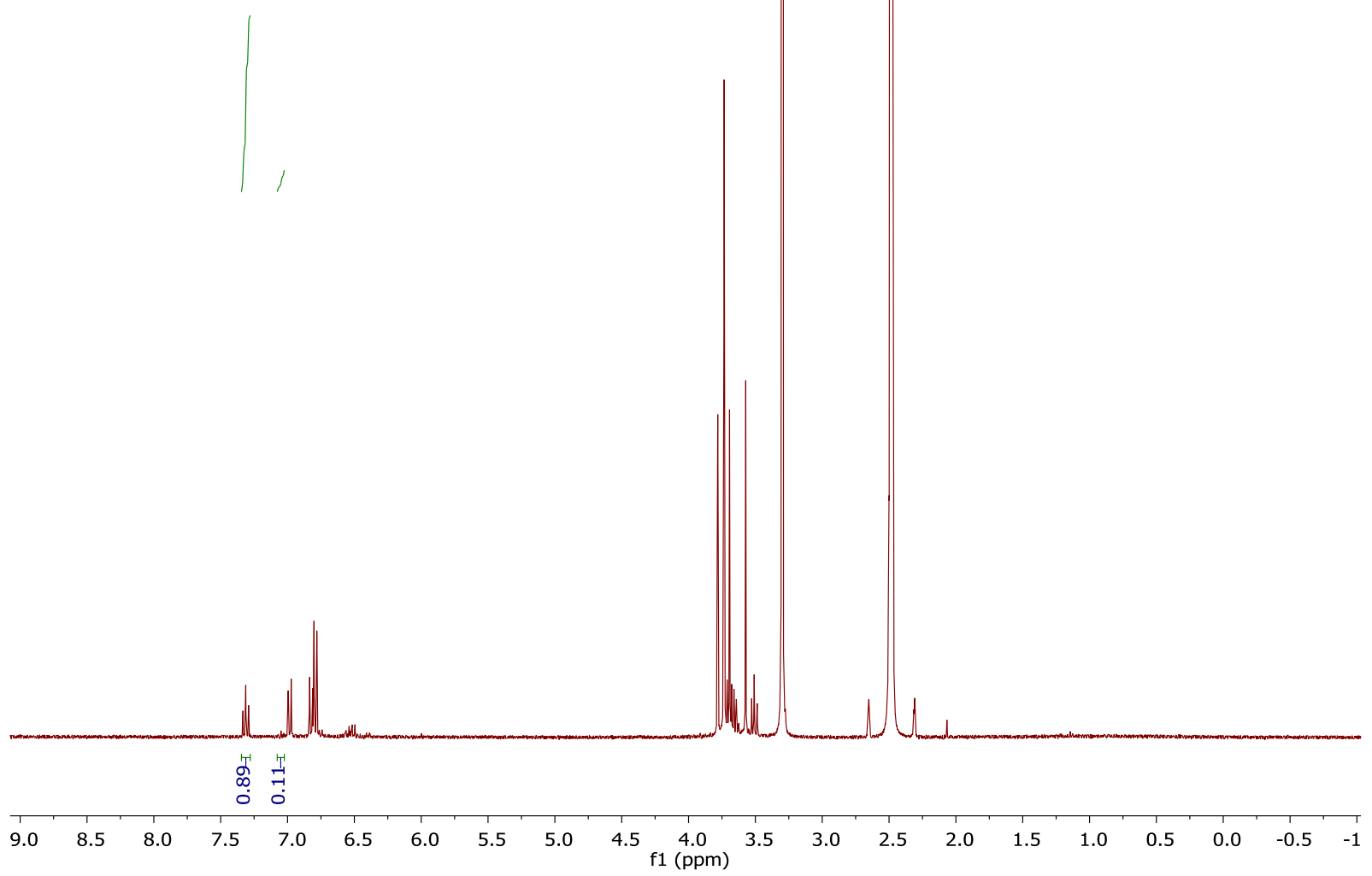

3k (PSS 365 nm irradiation):

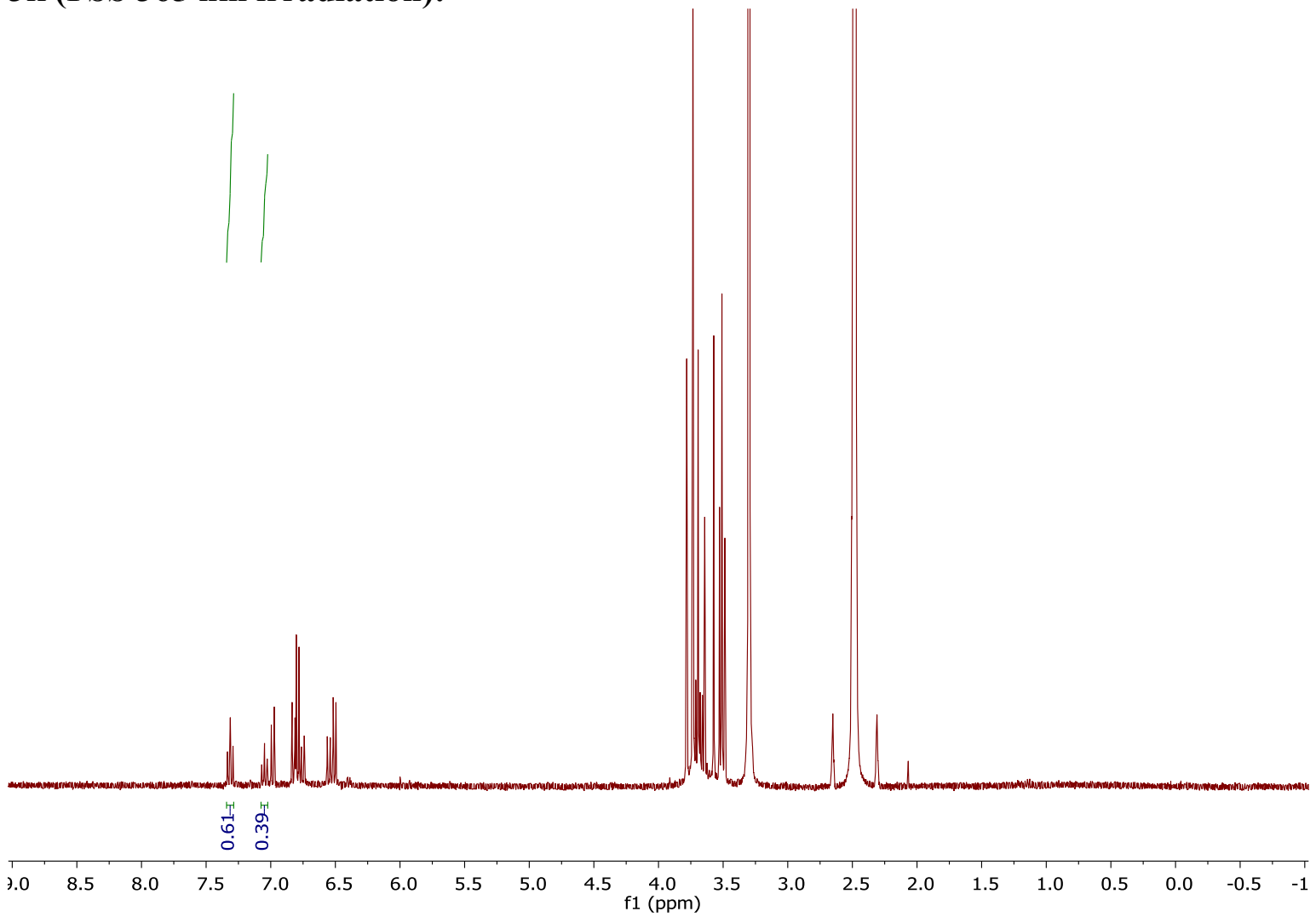


3k (PSS $530 \mathrm{~nm}$ irradiation):

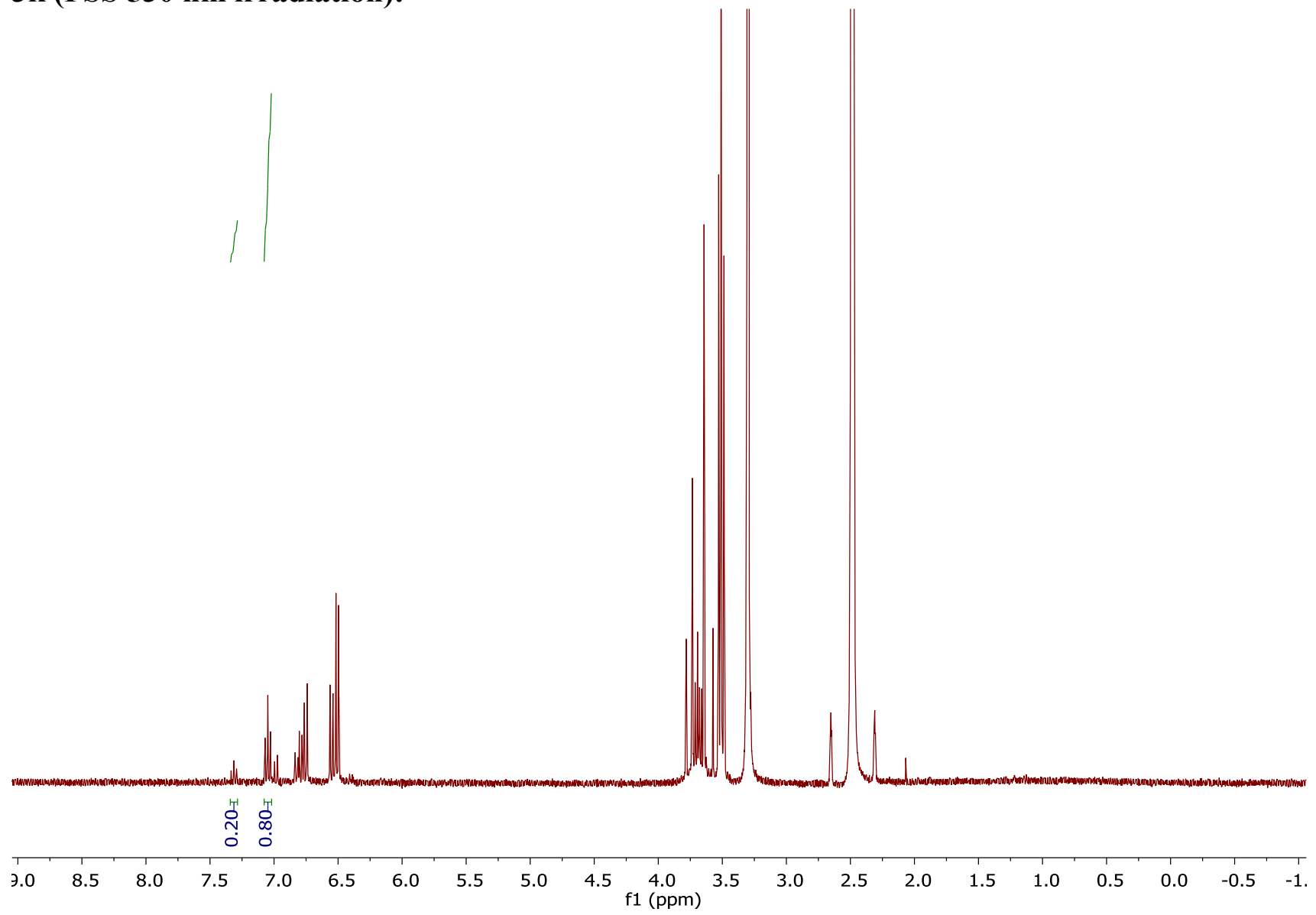




\section{${ }^{1} \mathrm{H},{ }^{13} \mathrm{C}-\mathrm{NMR}$ (DMSO-d 6 ), HR-MS (ESI+) and IR (neat) spectra}

2a:
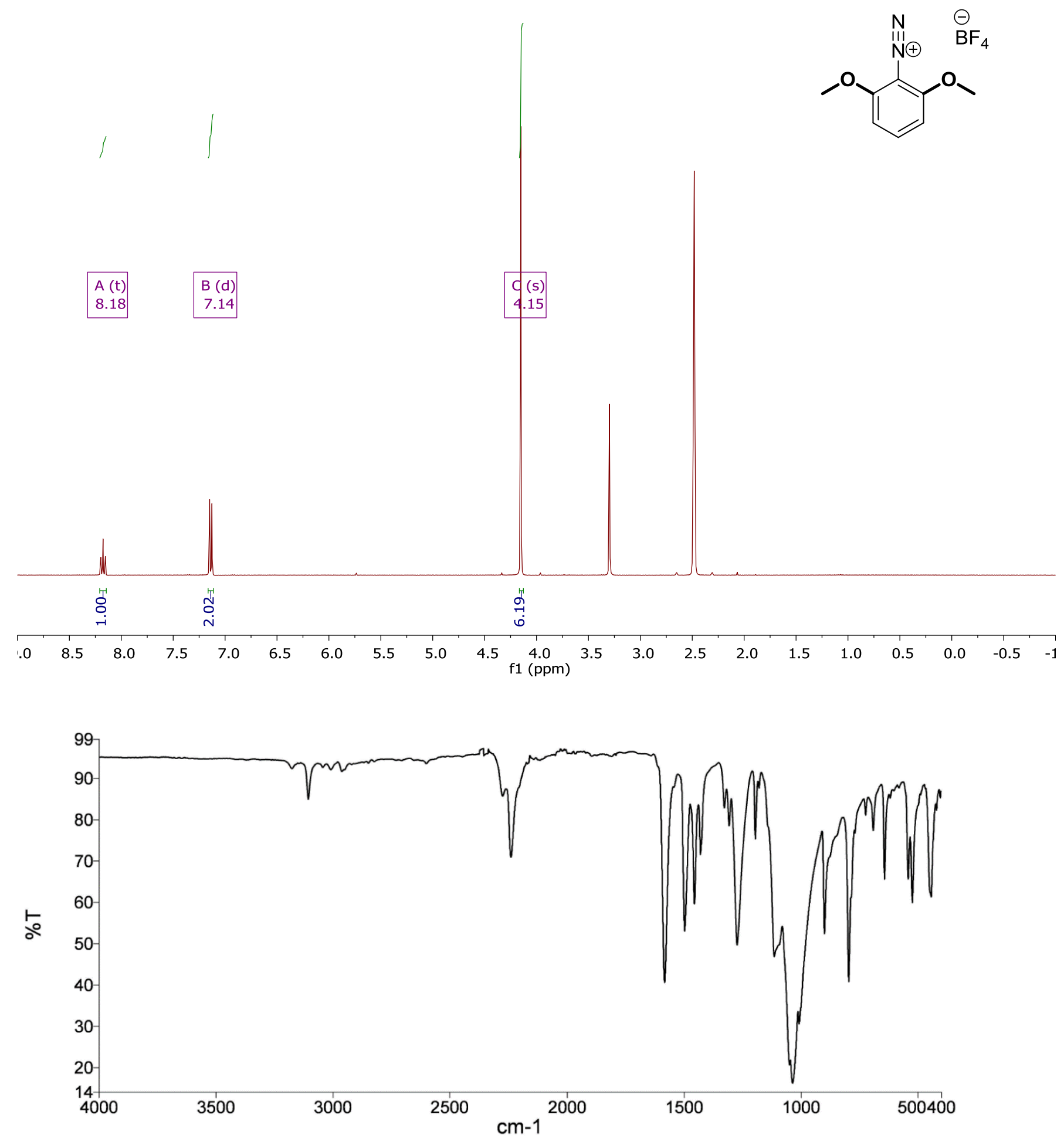
2b:
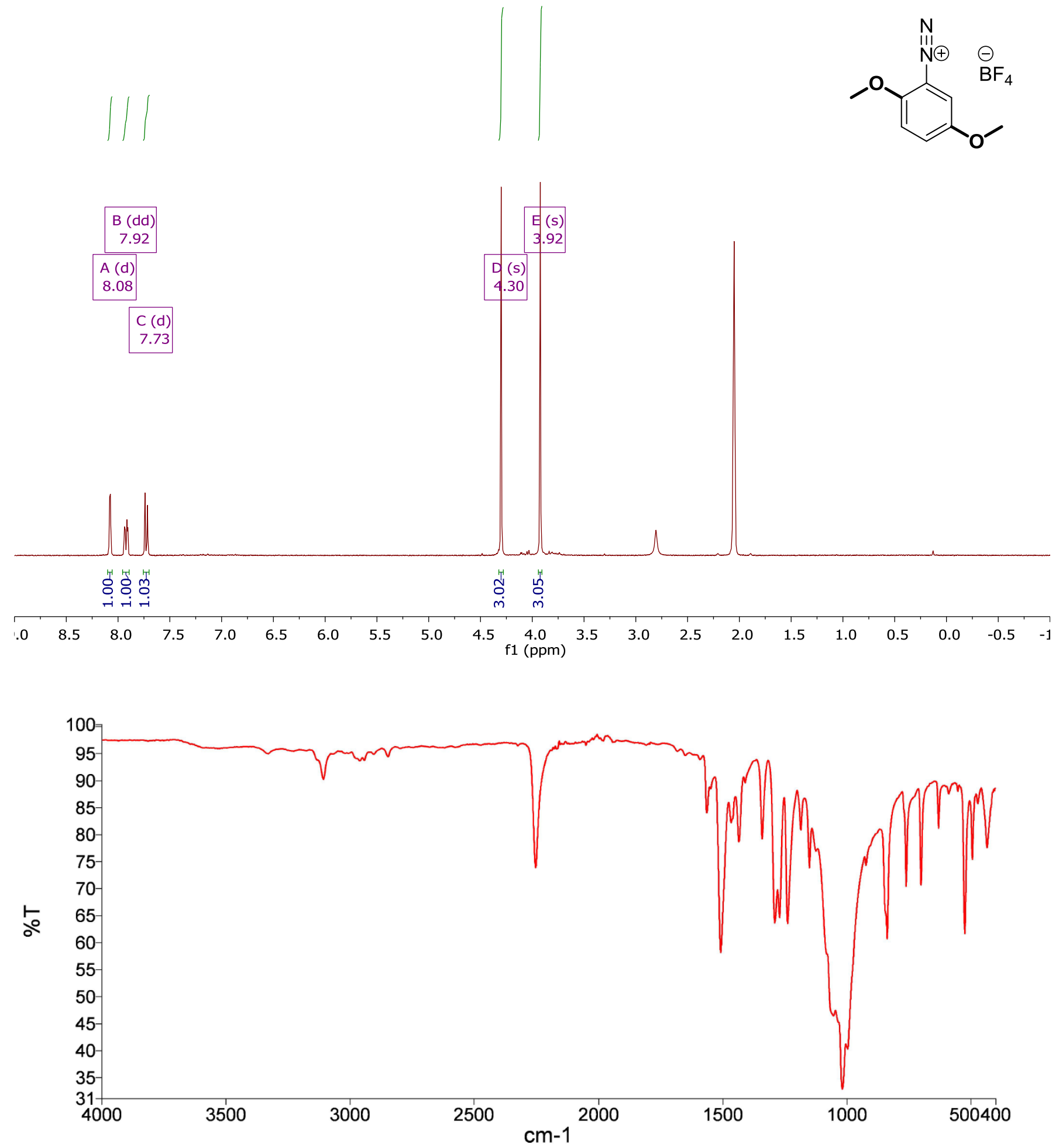
2d:
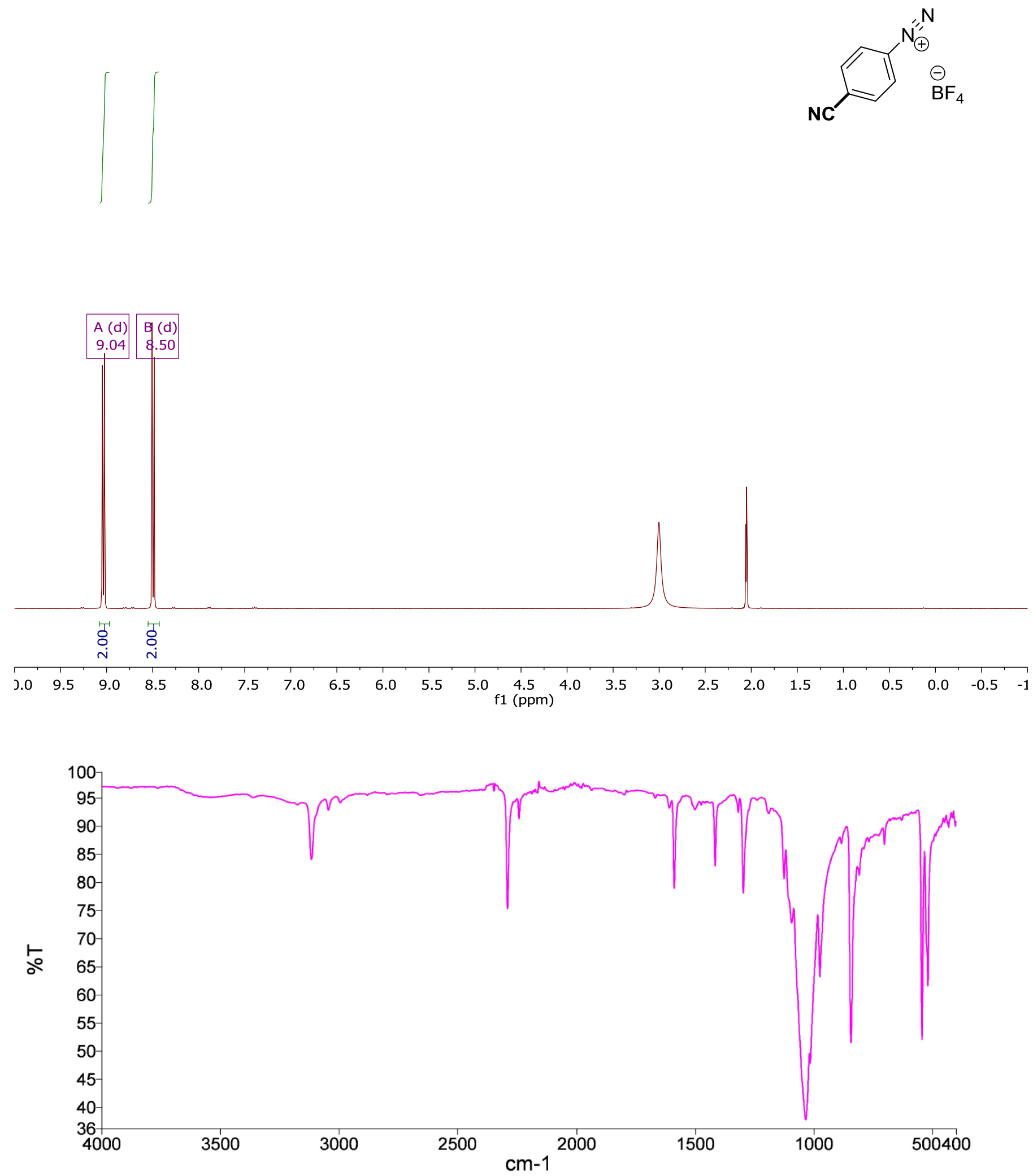
2e:
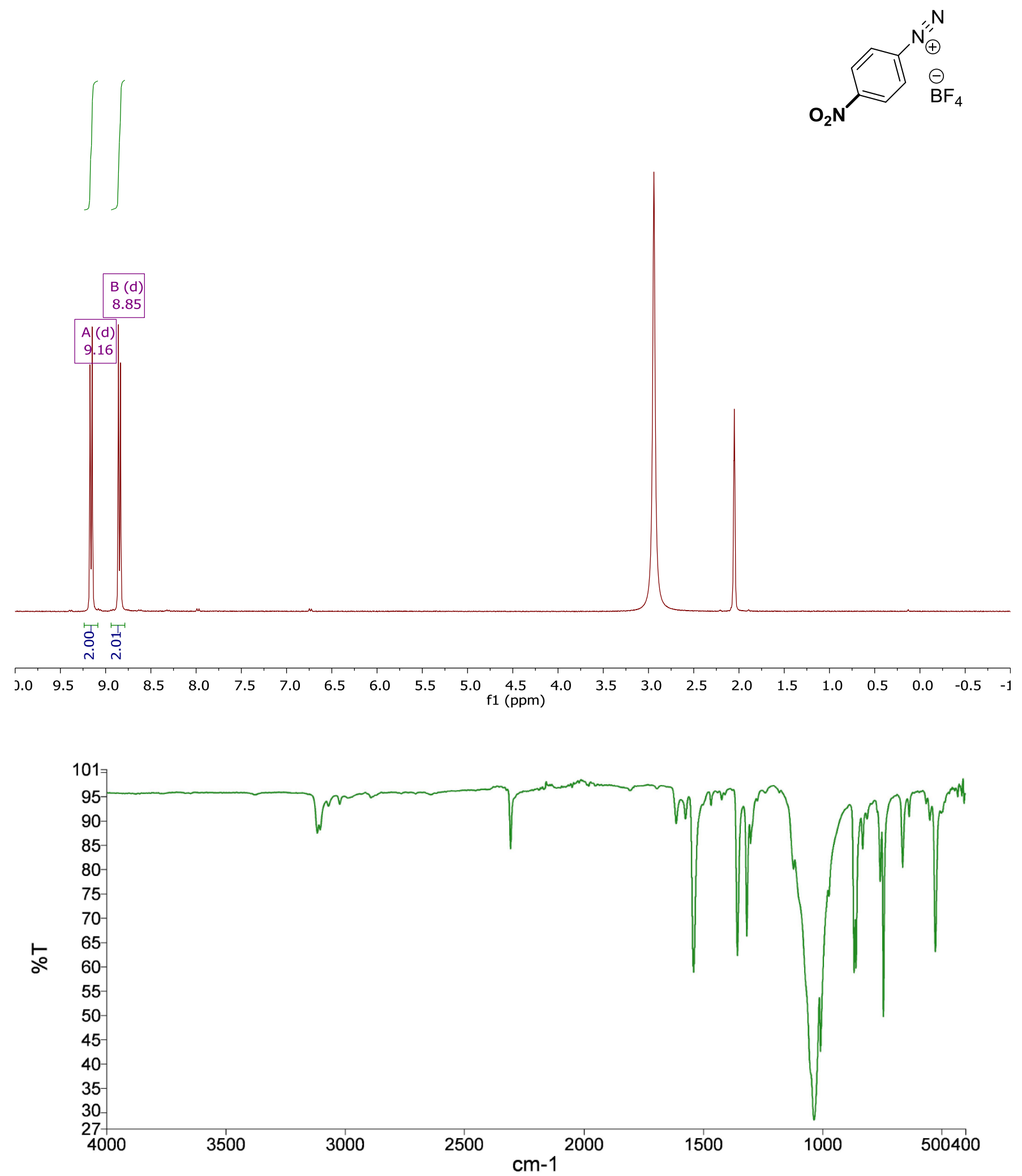
2f:
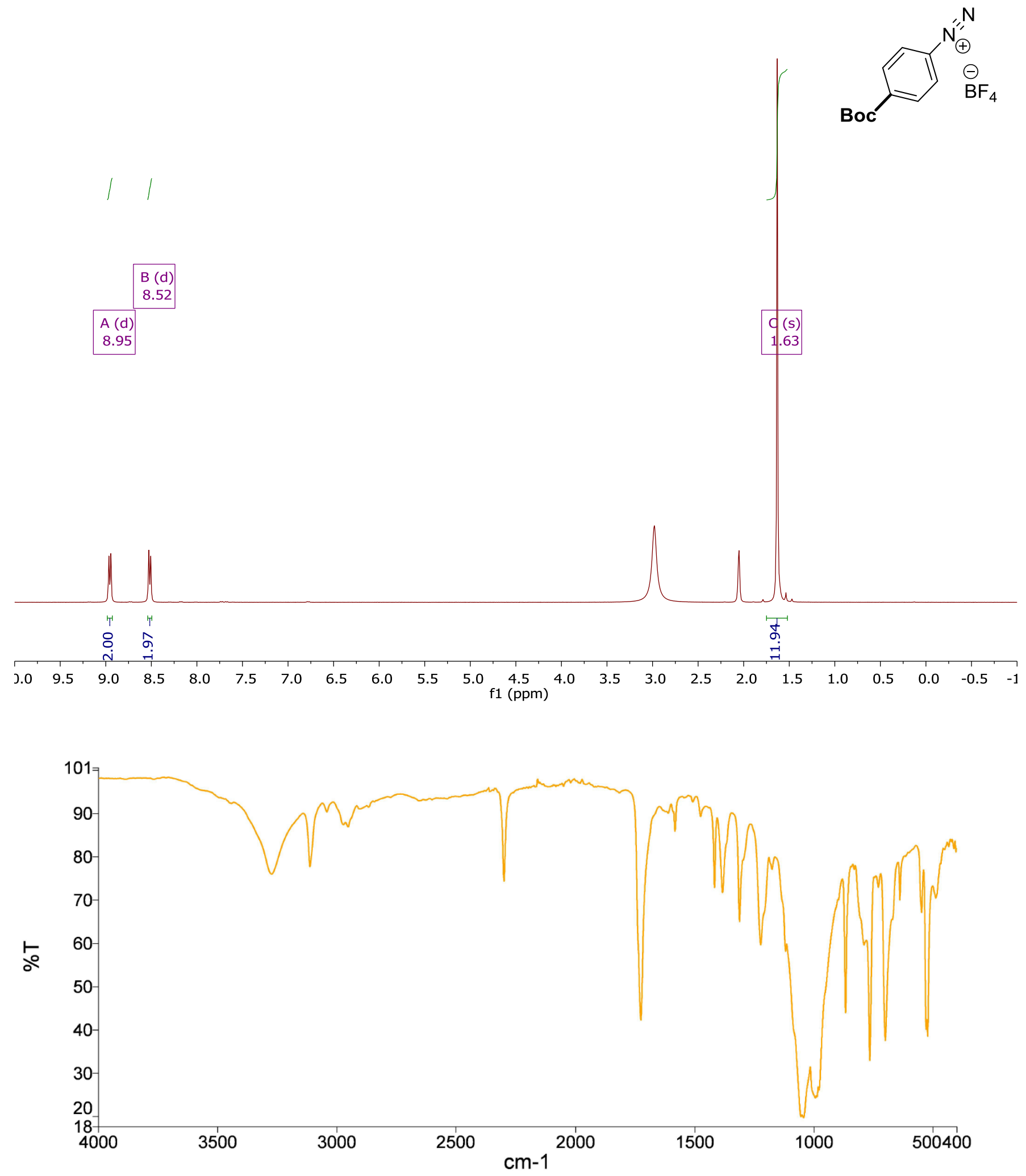
2g:

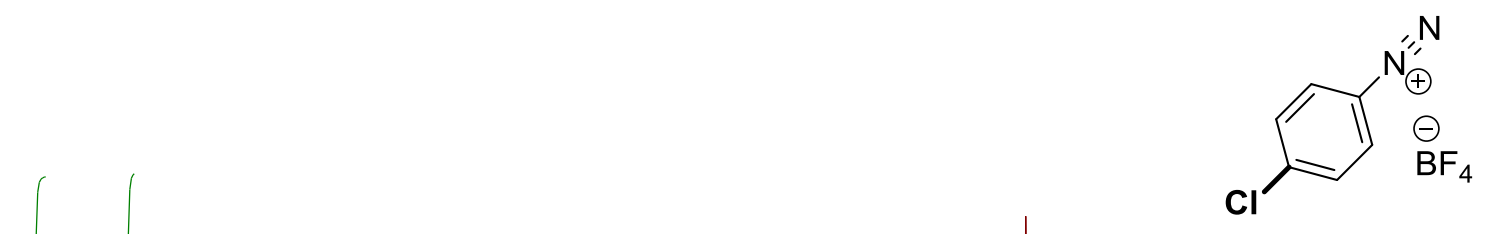

\section{गु่ ले}

\begin{tabular}{|c|c|c|c|c|c|c|c|c|c|c|c|c|c|c|c|c|c|c|c|c|c|}
\hline 3.0 & 9.5 & 9.0 & 8.5 & 8.0 & 7.5 & 7.0 & 6.5 & 6.0 & 5.5 & 5.0 & $\begin{array}{c}4.5 \\
1(\mathrm{ppm}\end{array}$ & 4.0 & 3.5 & 3.0 & 2.5 & 2.0 & 1.5 & 1.0 & 0.5 & 0.0 & -0.5 \\
\hline
\end{tabular}

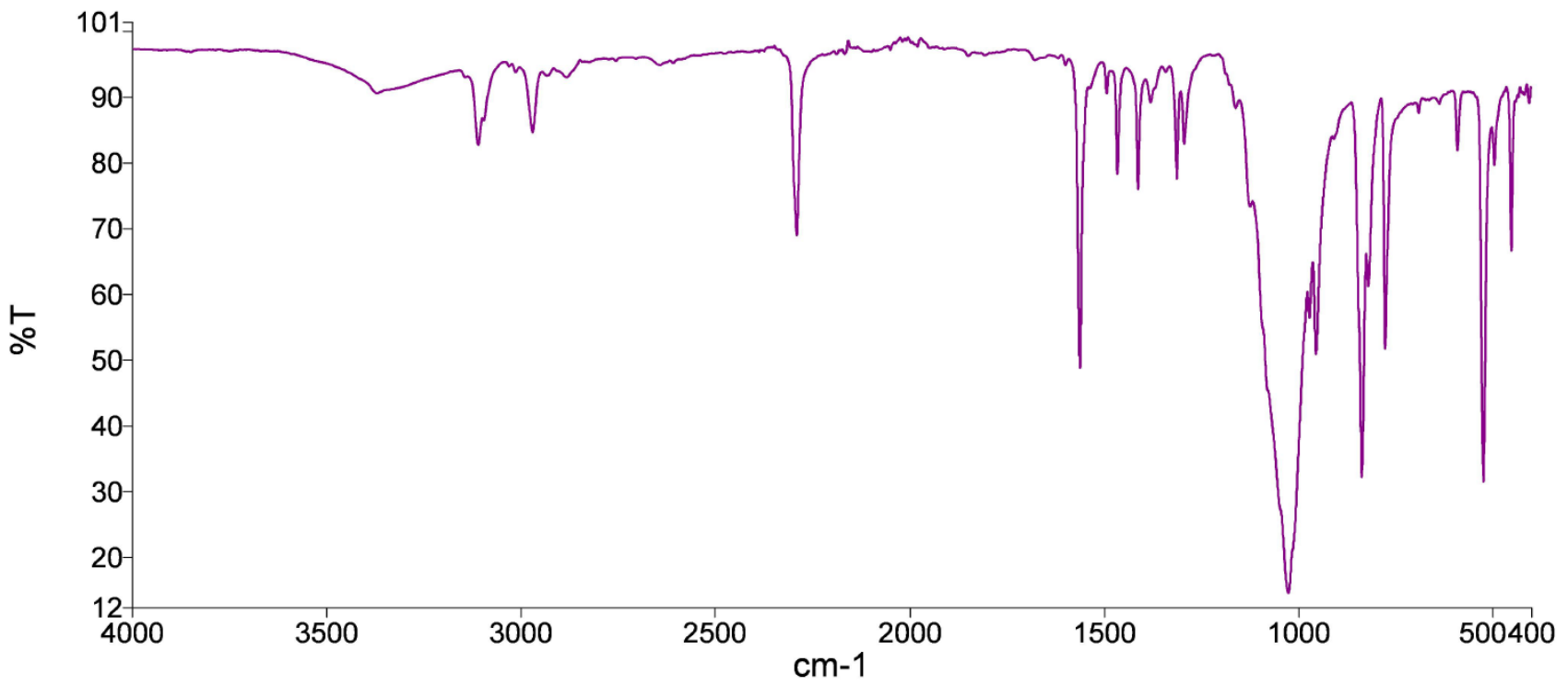


2h:
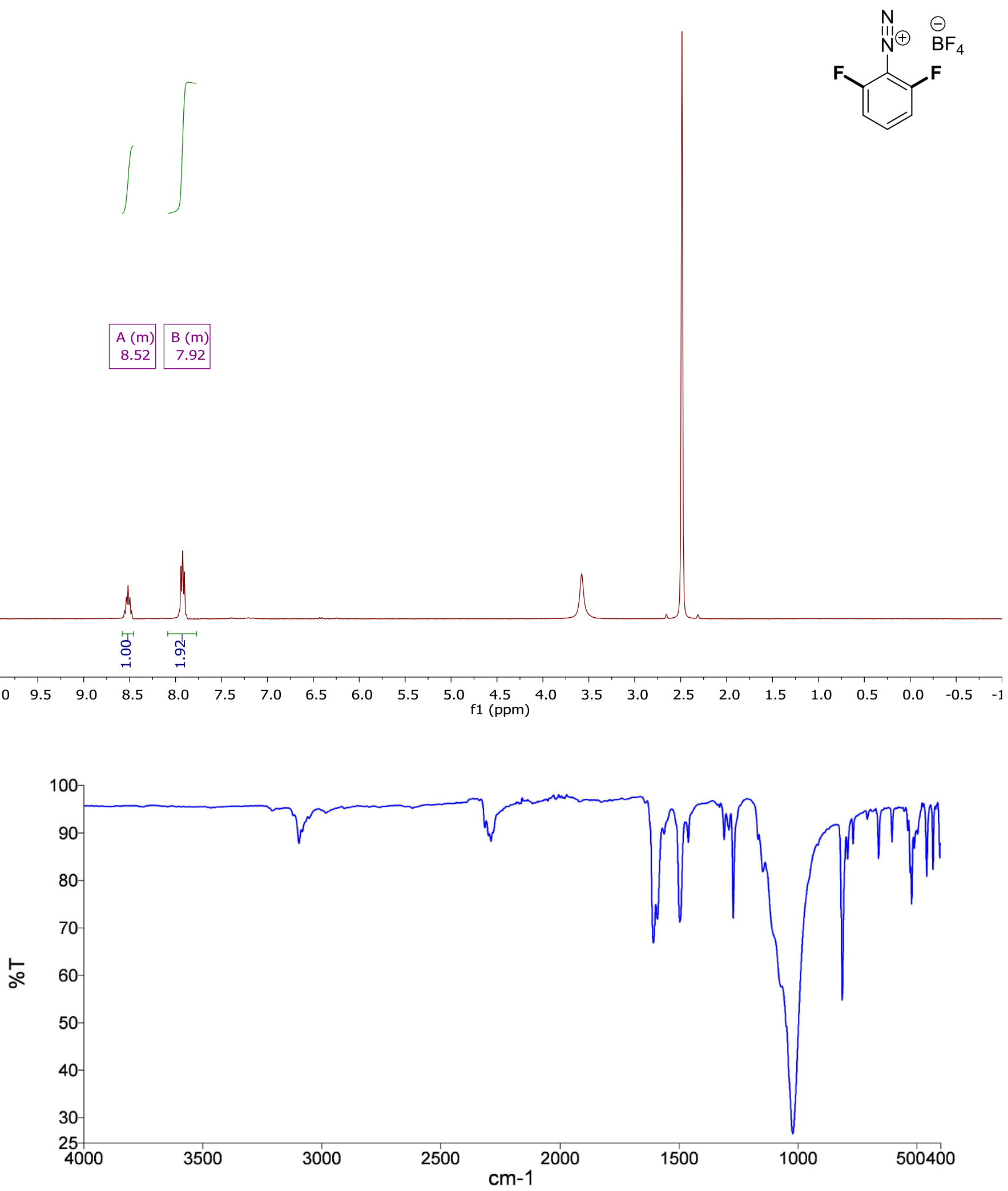
2i:
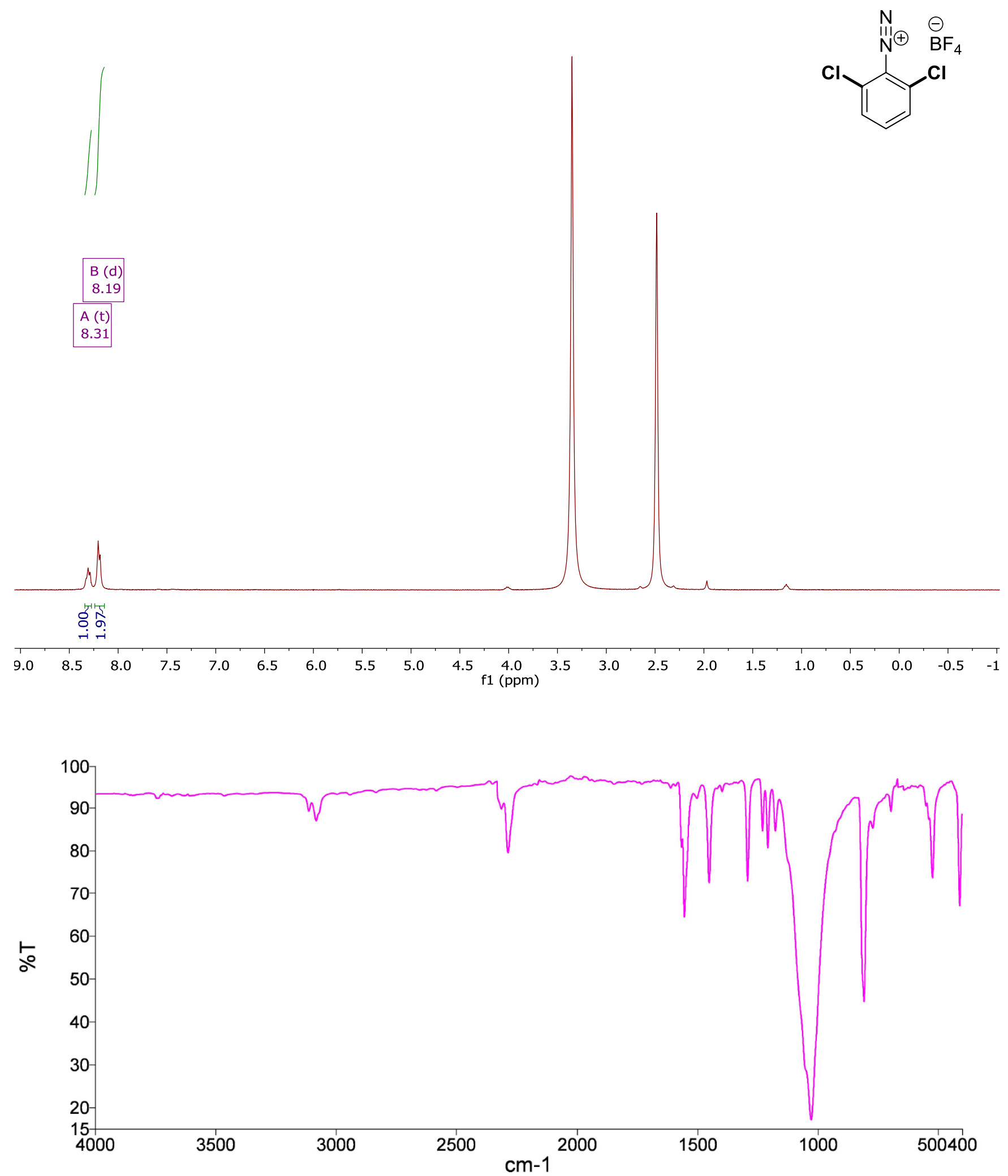
2j: Upon preparation of the NMR sample, some hydrolysis of the pinacol boronic ester was observed, which becomes apparent in the ${ }^{1} \mathrm{H}-\mathrm{NMR}$ spectrum at 8.4 and $7.8 \mathrm{ppm}$.
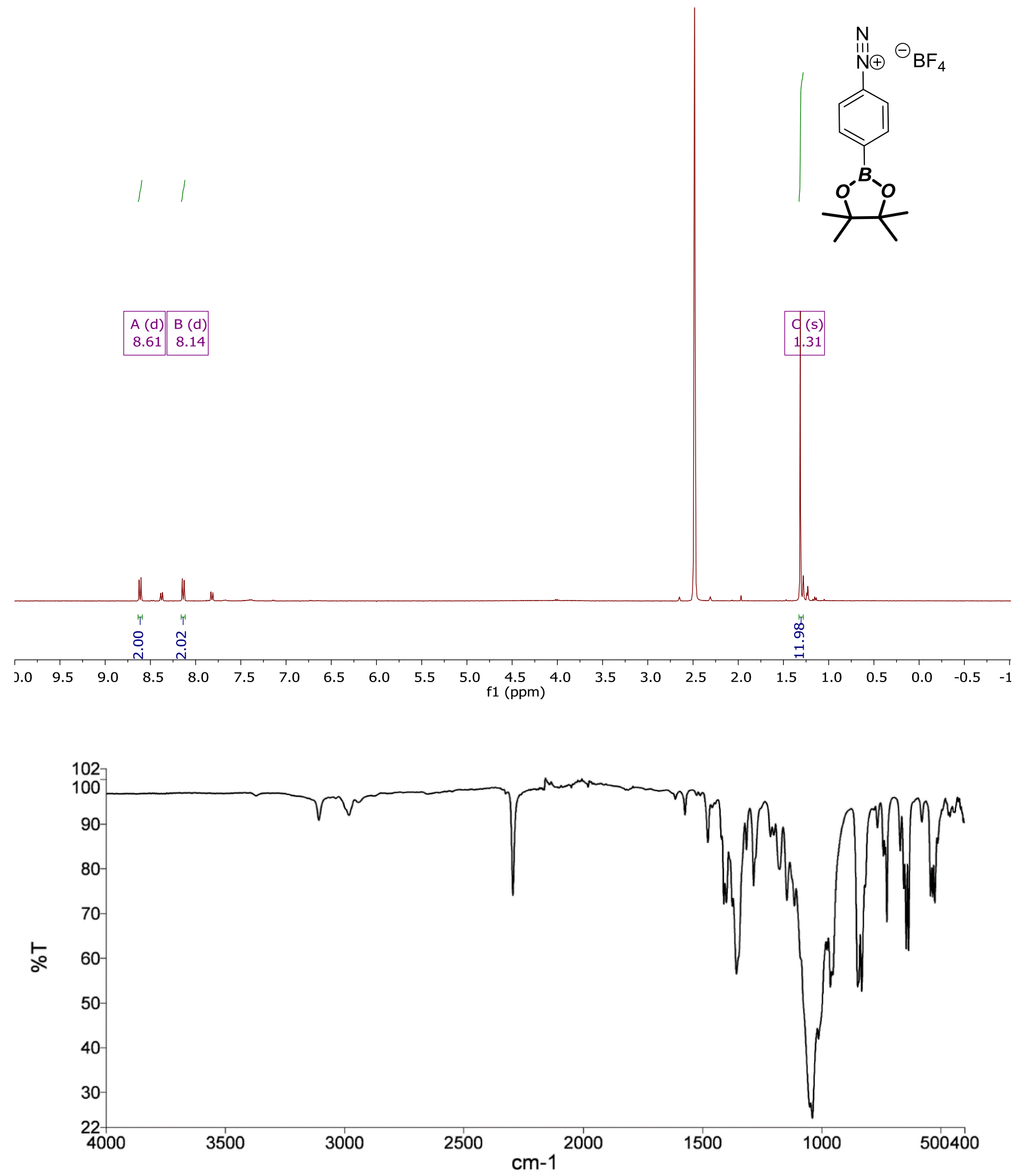
3a:

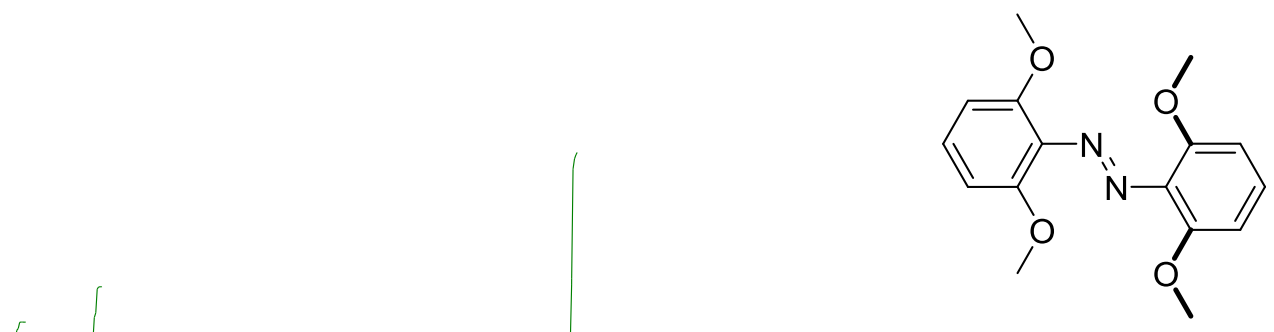

\begin{tabular}{l|l}
$A(t)$ & $B(d)$ \\
7.28 & 6.77
\end{tabular}

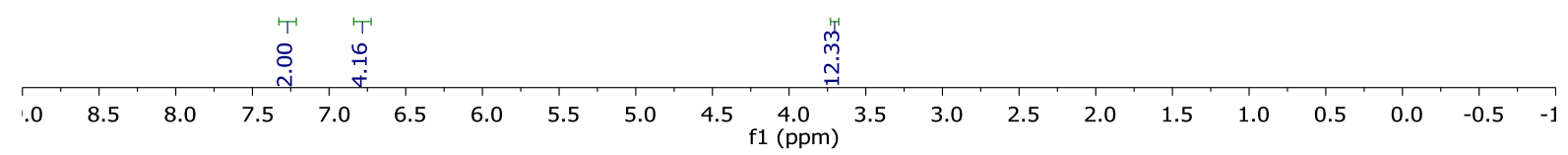

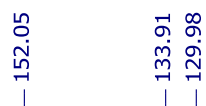

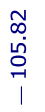

$\stackrel{8}{\circ}$

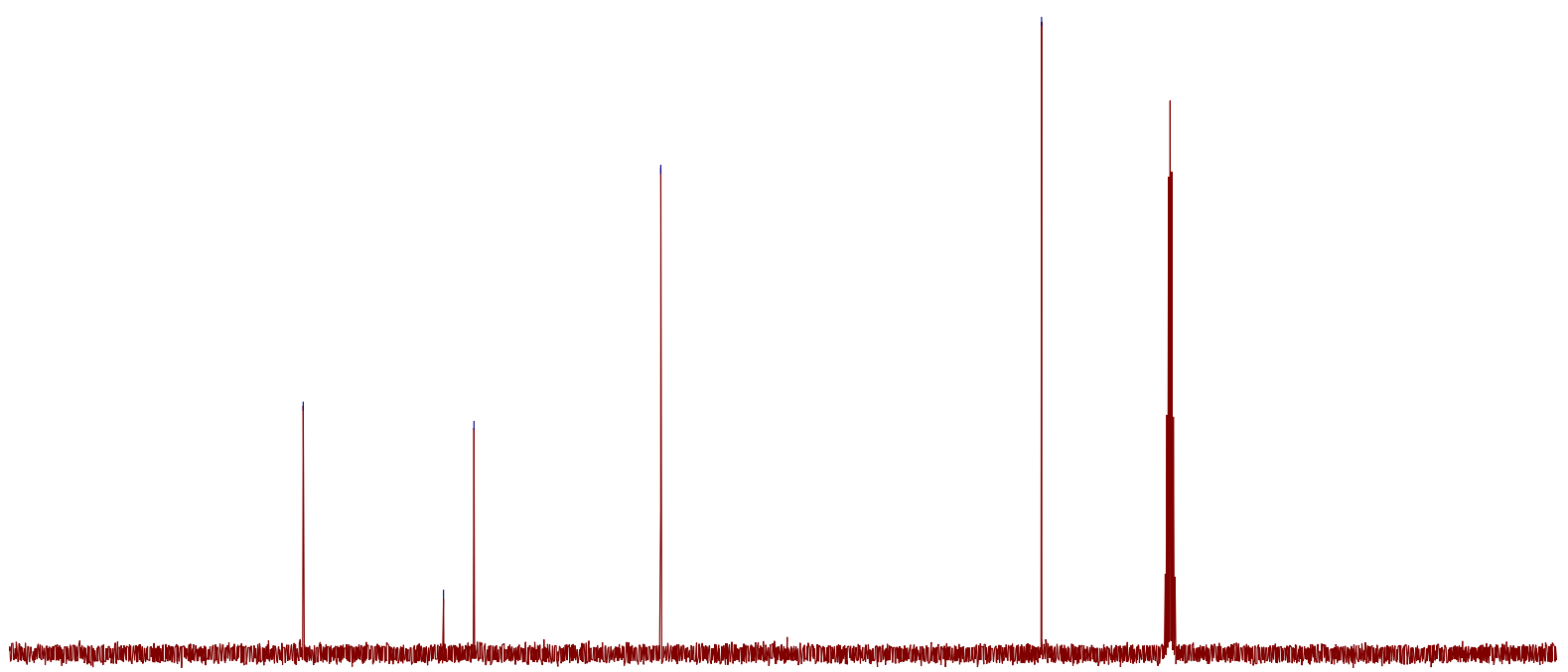

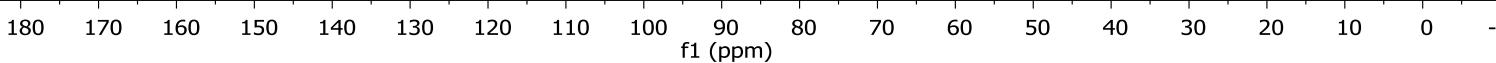




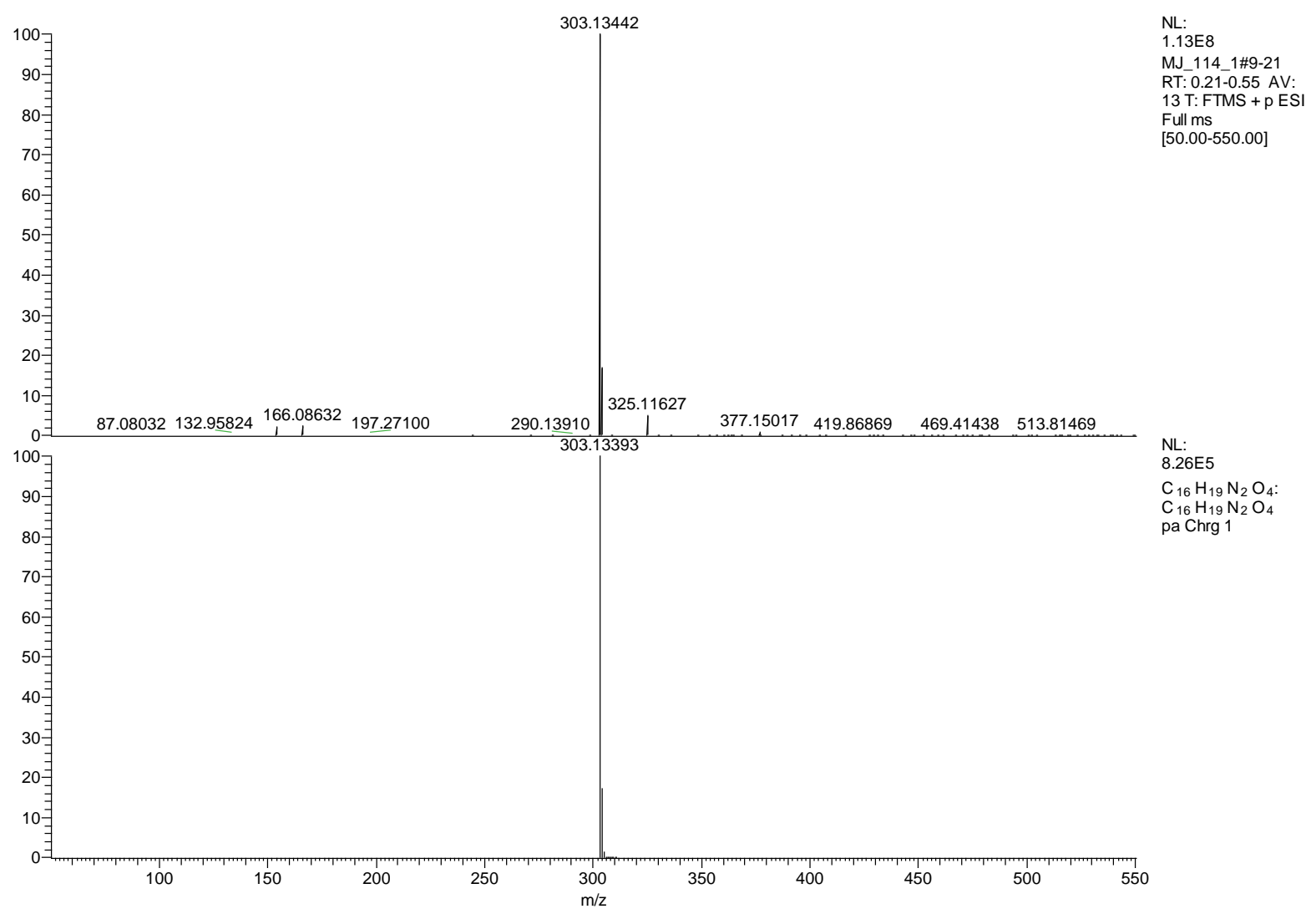

3b: After thermal adaption, no full conversion to the trans isomer was observed which became apparent in the ${ }^{1} \mathrm{H}-\mathrm{NMR}$ and ${ }^{13} \mathrm{C}-\mathrm{NMR}$ spectrum showing small quantities of the cis isomer.
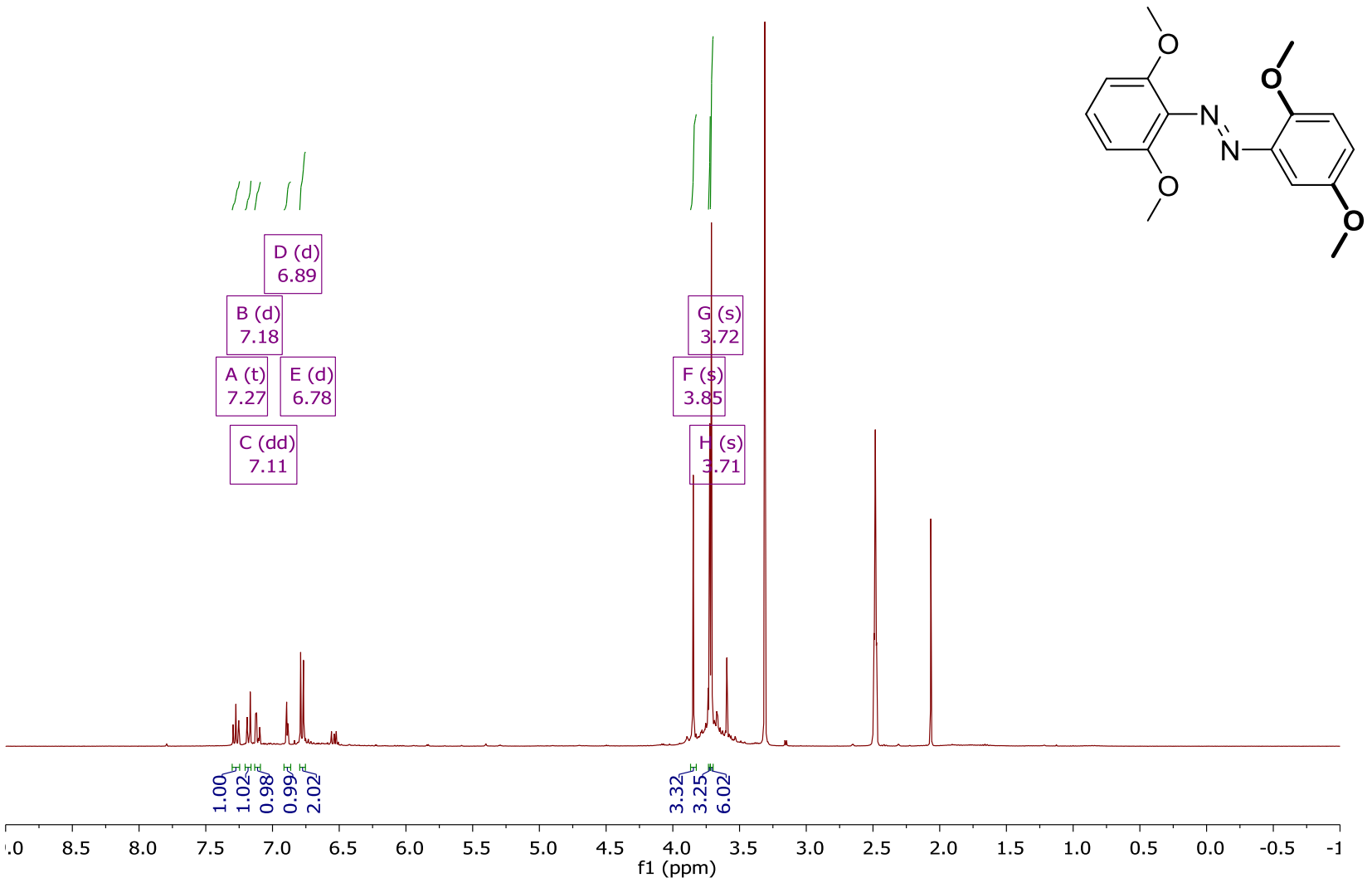


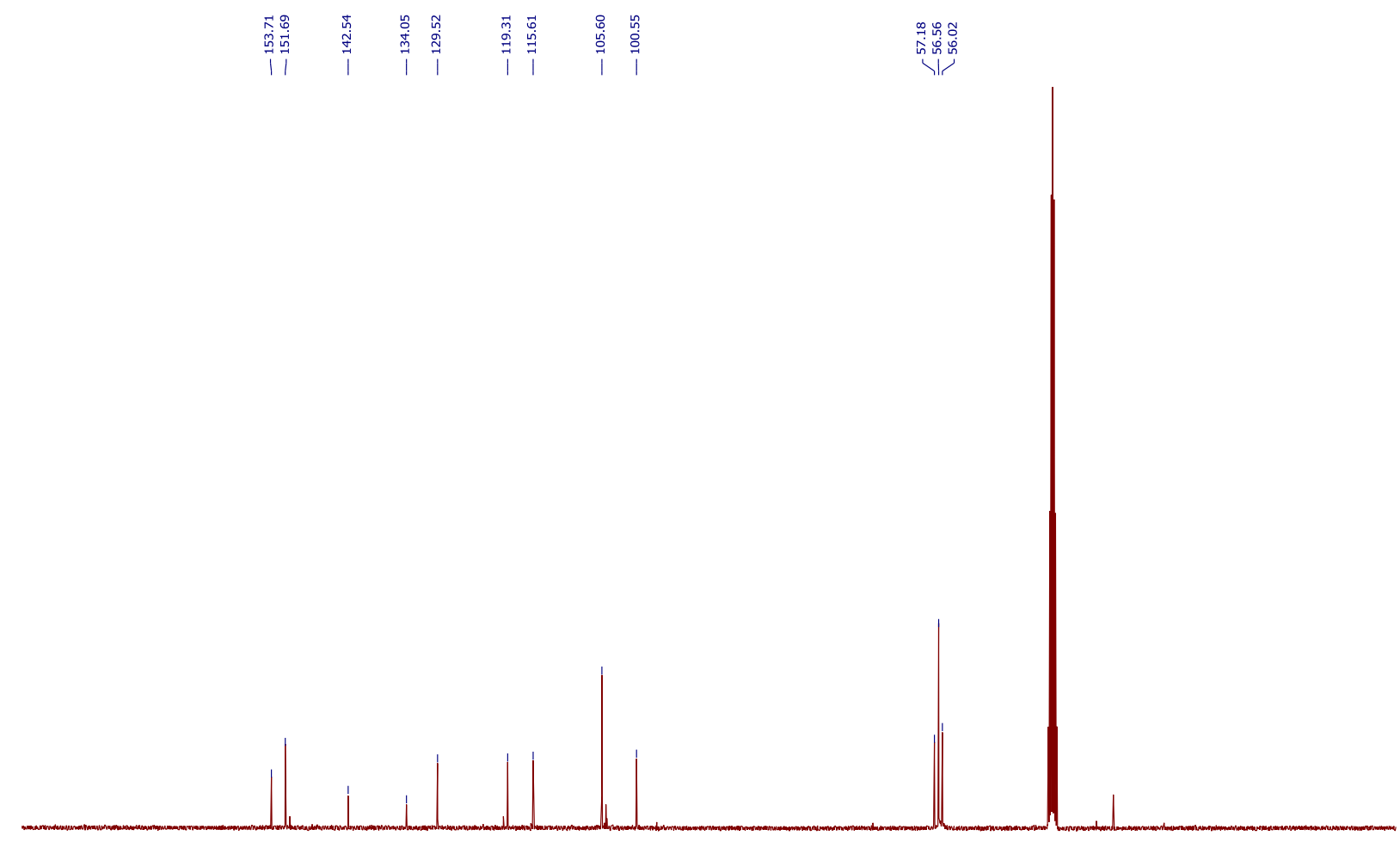

$\begin{array}{lllllllllllllllllllll}90 & 180 & 170 & 160 & 150 & 140 & 130 & 120 & 110 & 100 & \begin{array}{c}90 \\ \mathrm{f} 1(\mathrm{ppm})\end{array} & 80 & 70 & 60 & 50 & 40 & 30 & 20 & 10 & 0 & -\end{array}$

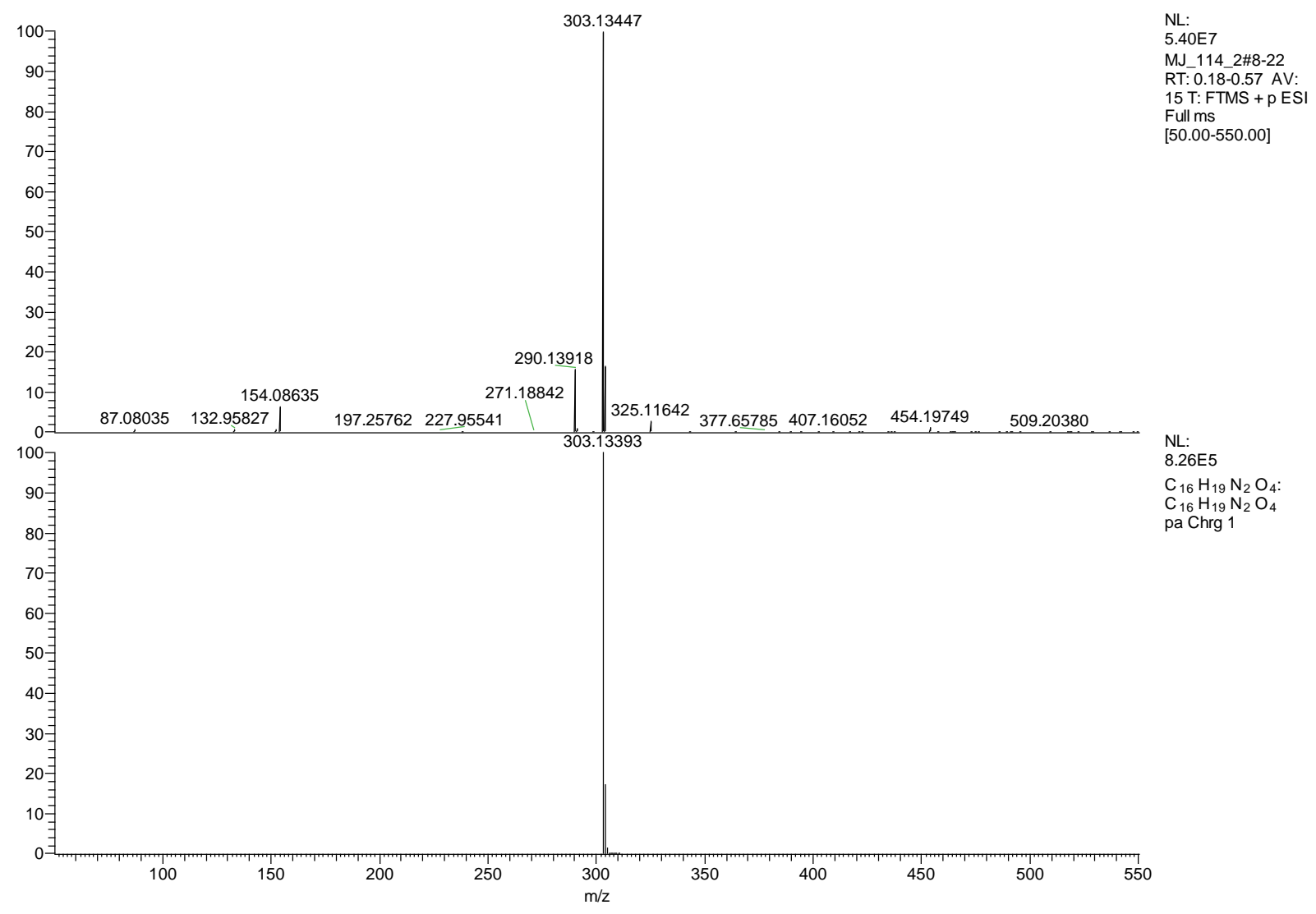


3c: After thermal adaption, no full conversion to the trans isomer was observed which became apparent in the ${ }^{1} \mathrm{H}-\mathrm{NMR}$ and ${ }^{13} \mathrm{C}-\mathrm{NMR}$ spectrum showing small quantities of the $c$ is isomer.
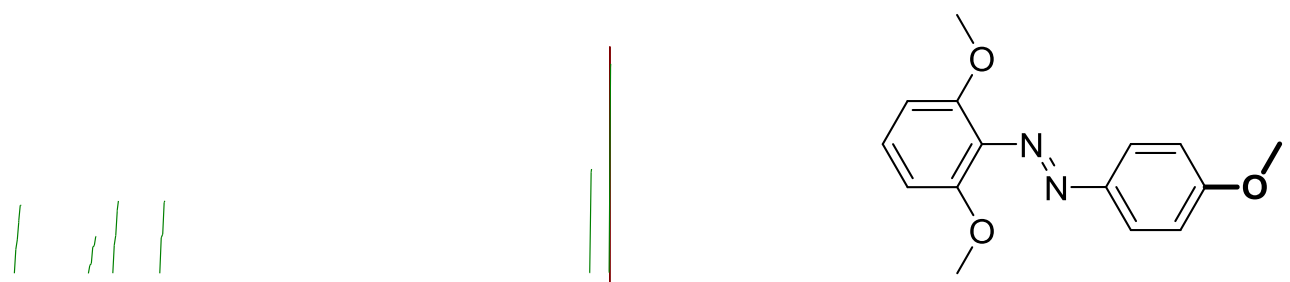

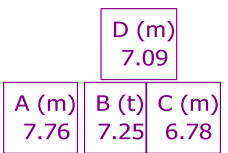
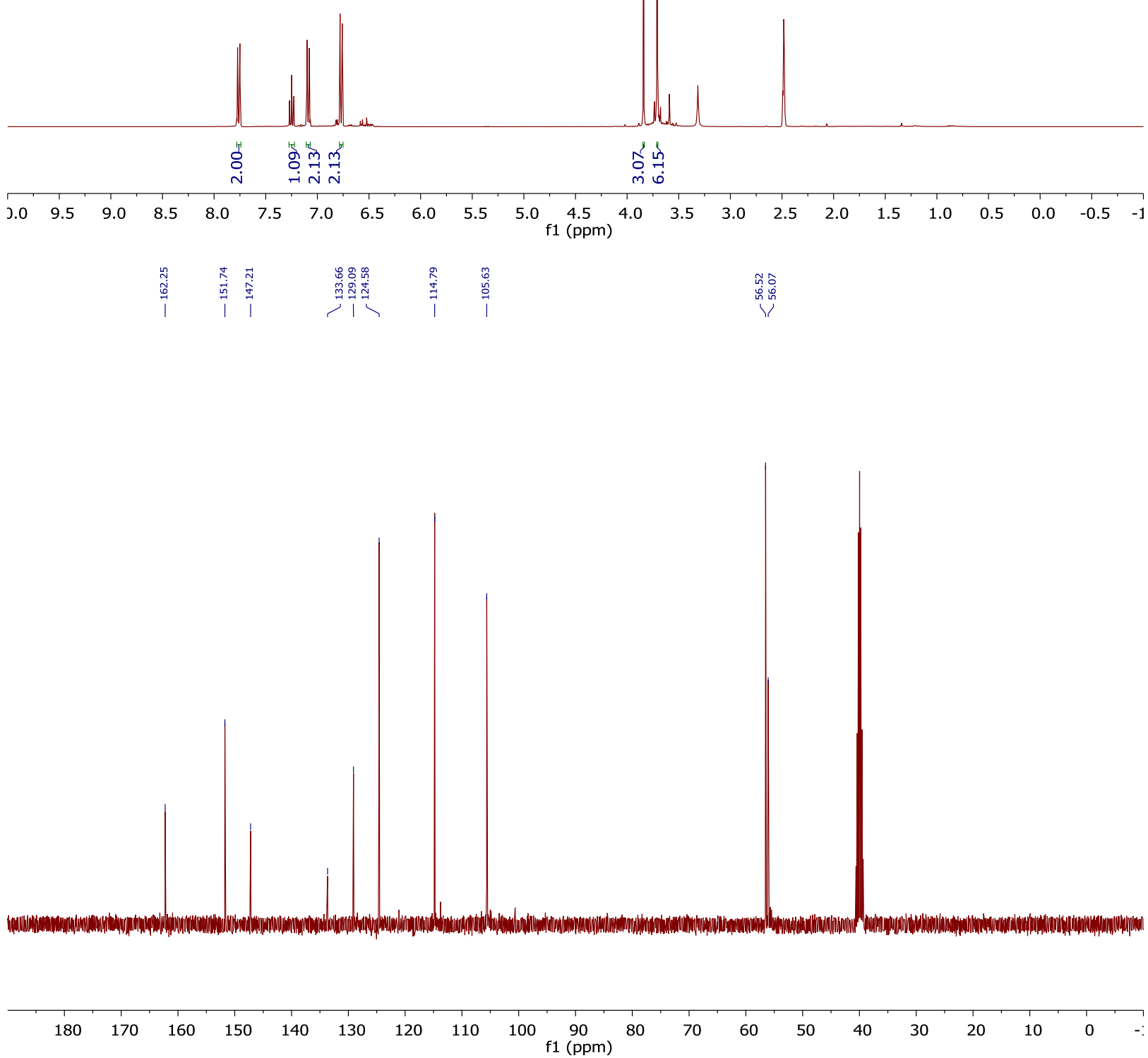


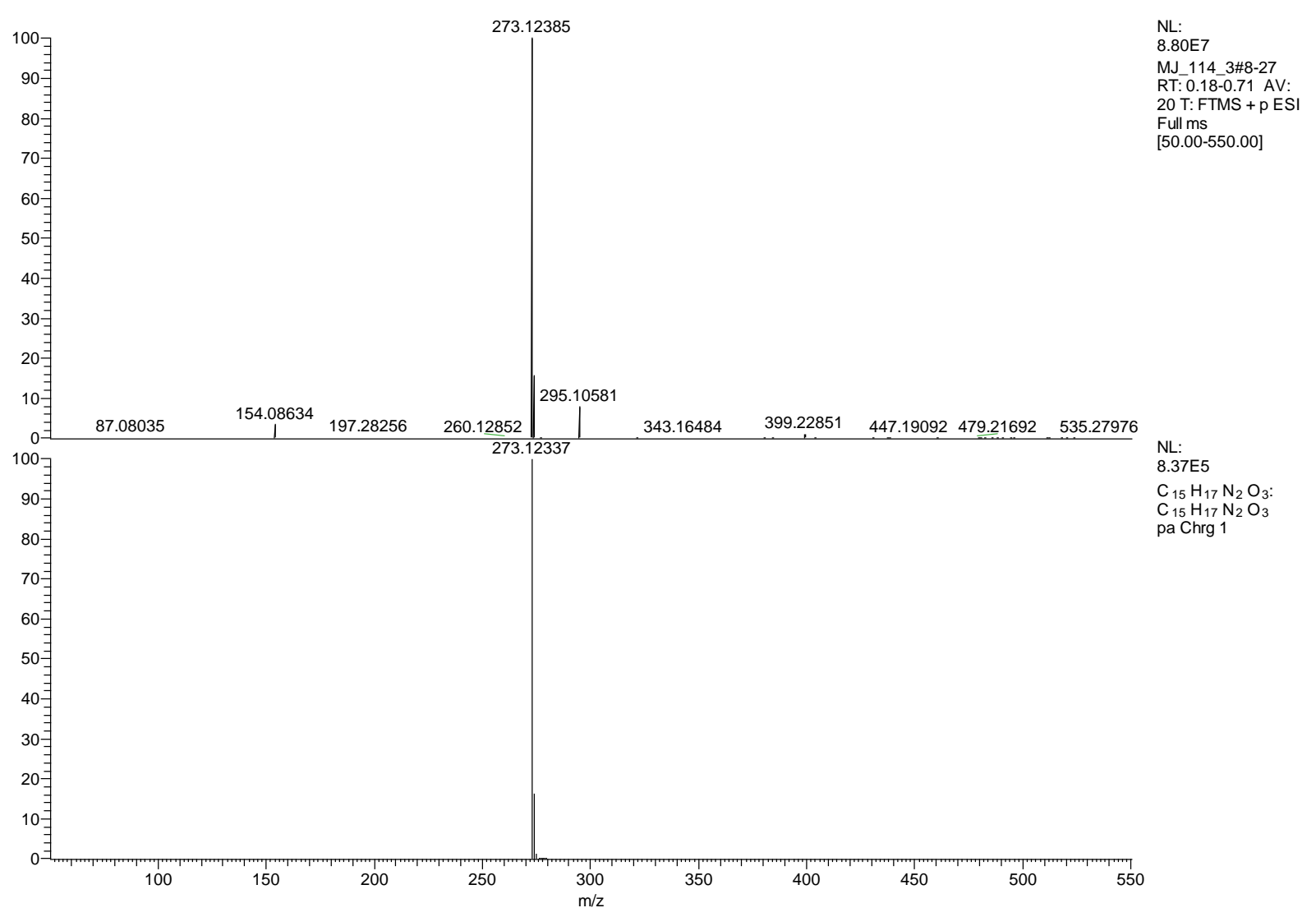

3d:

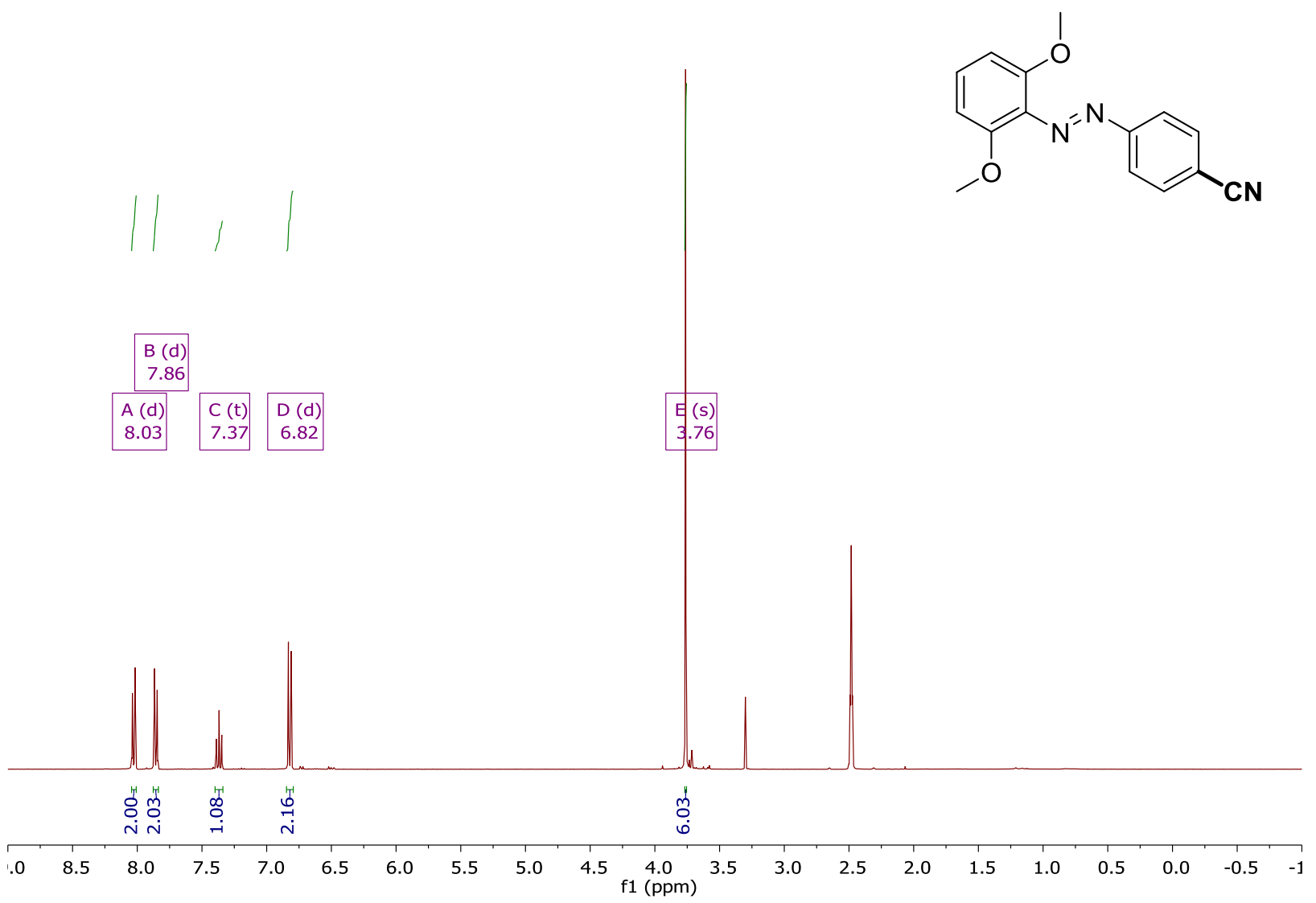



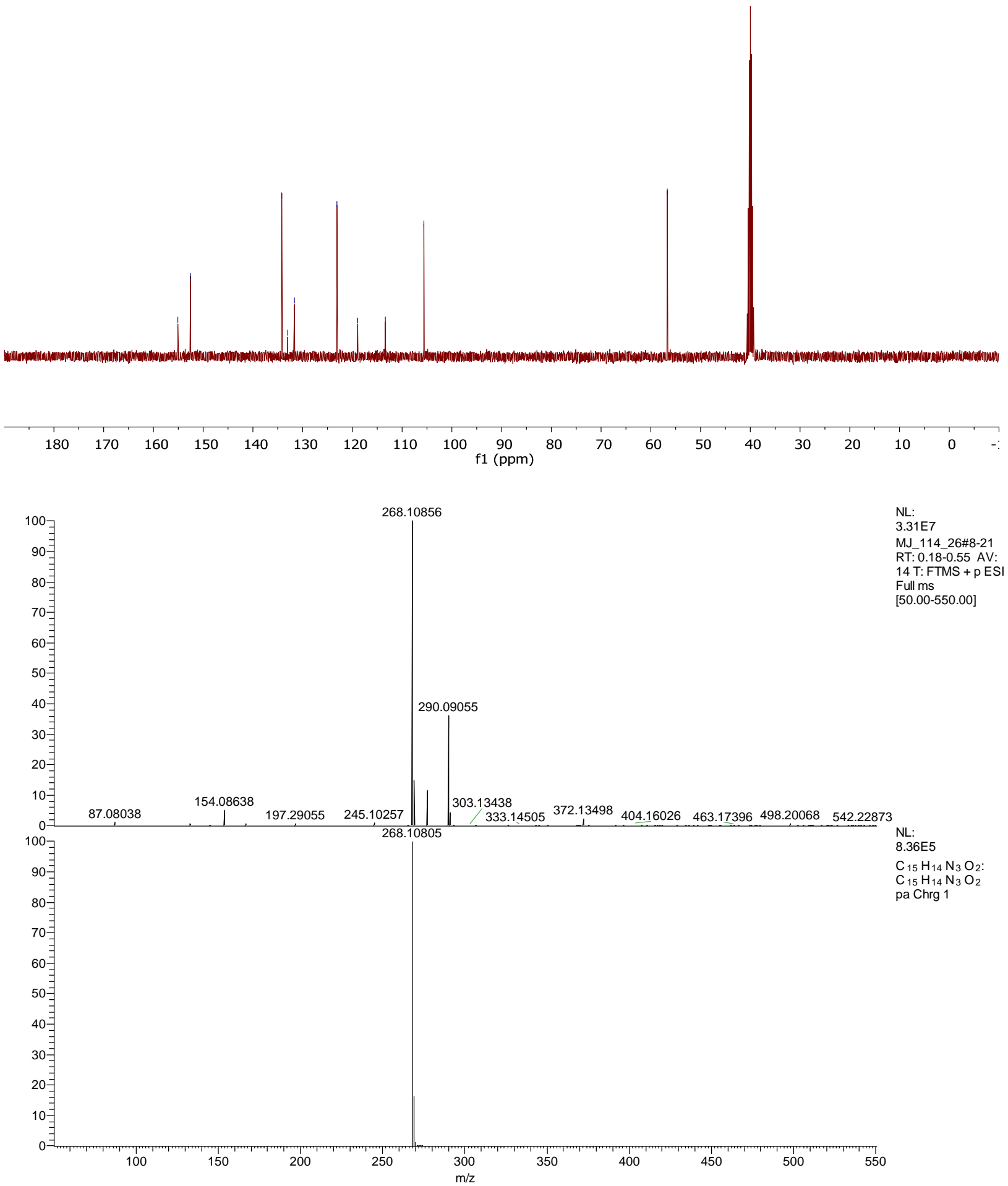
3e: After thermal adaption, no full conversion to the trans isomer was observed which became apparent in the ${ }^{1} \mathrm{H}-\mathrm{NMR}$ and ${ }^{13} \mathrm{C}-\mathrm{NMR}$ spectrum showing small quantities of the $\mathrm{cis}$ isomer.
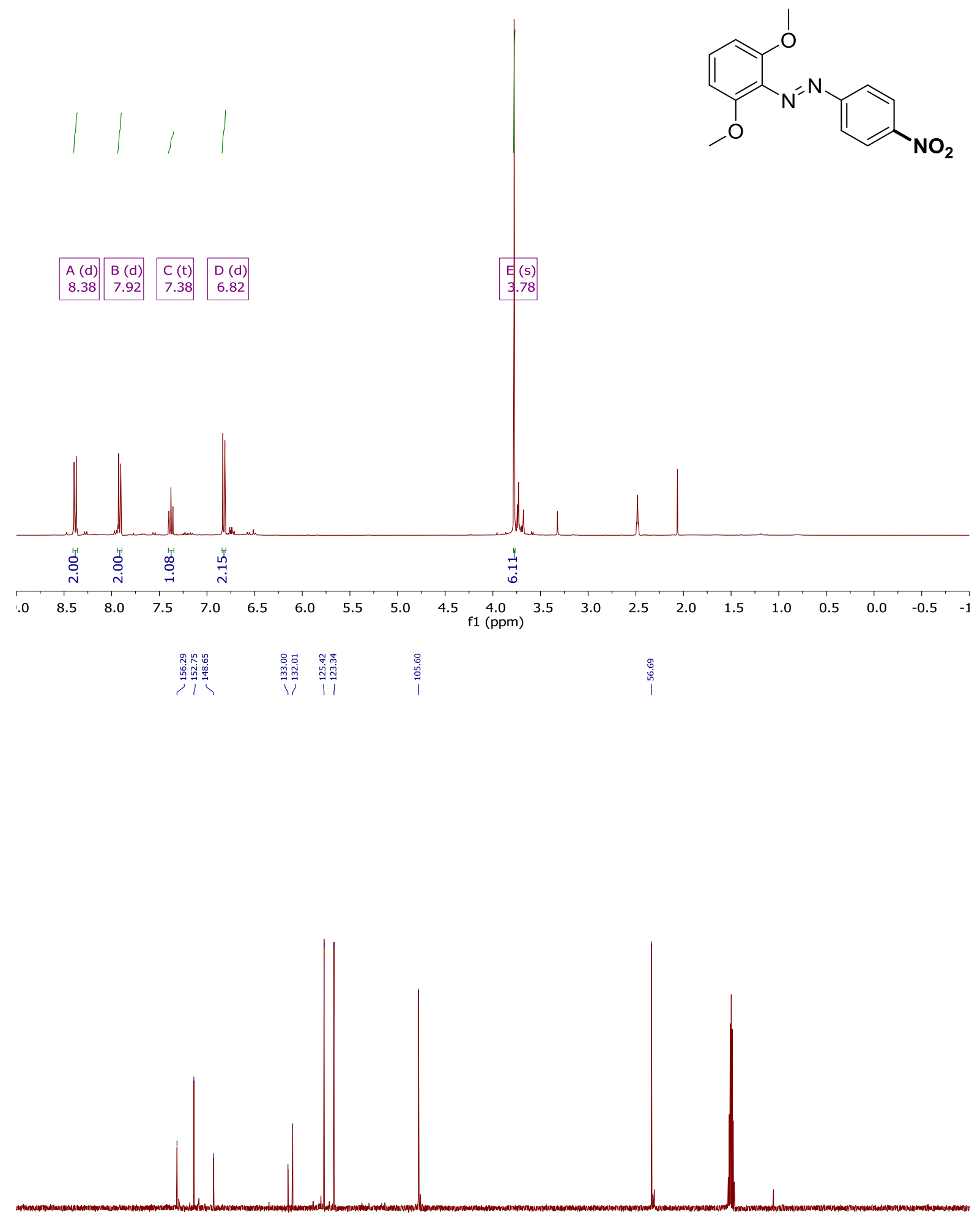

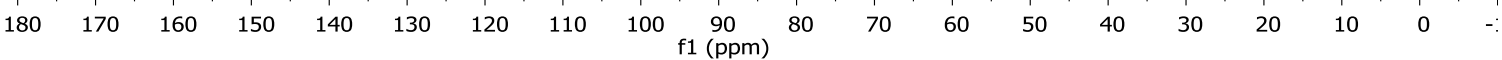




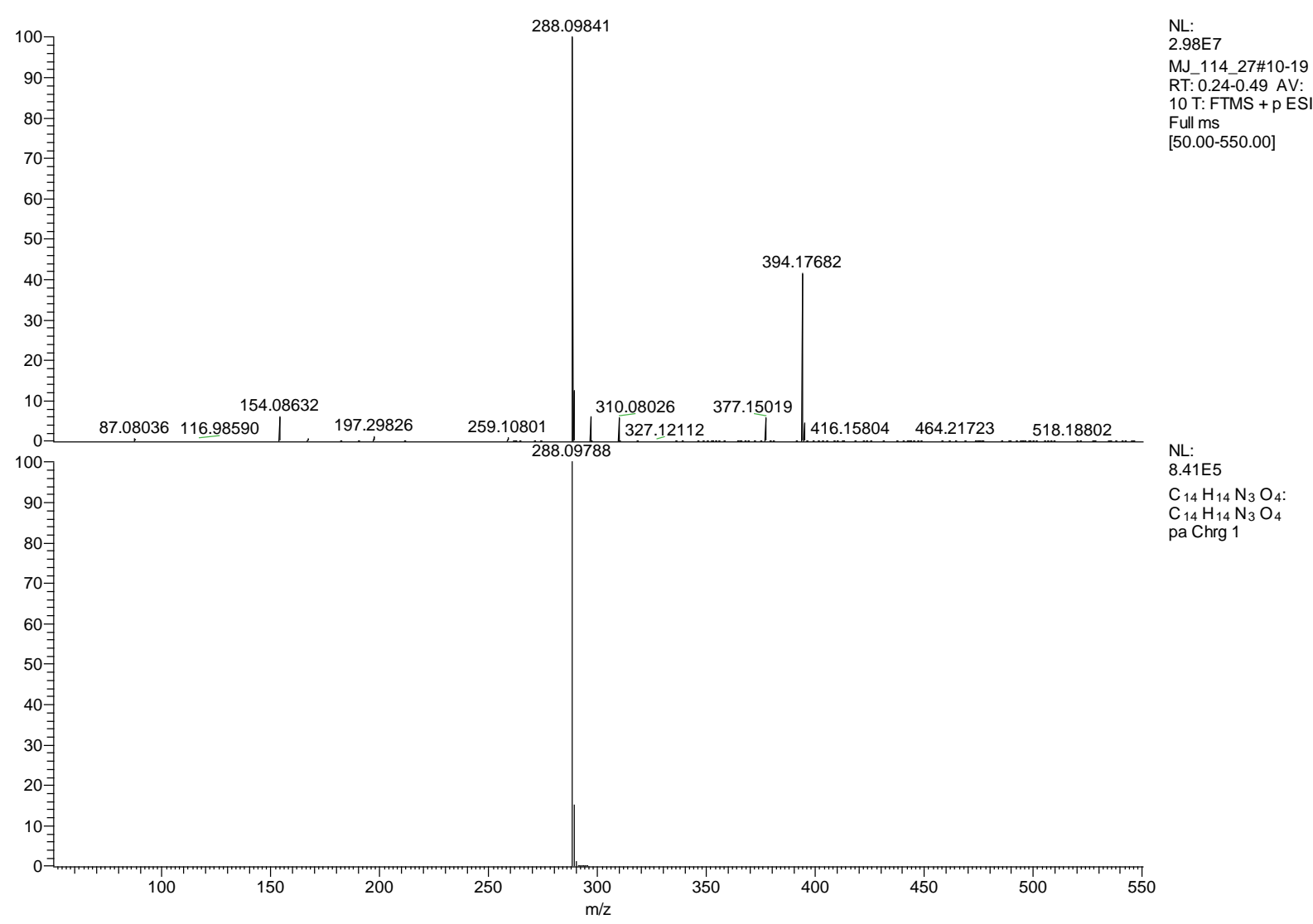

3f:

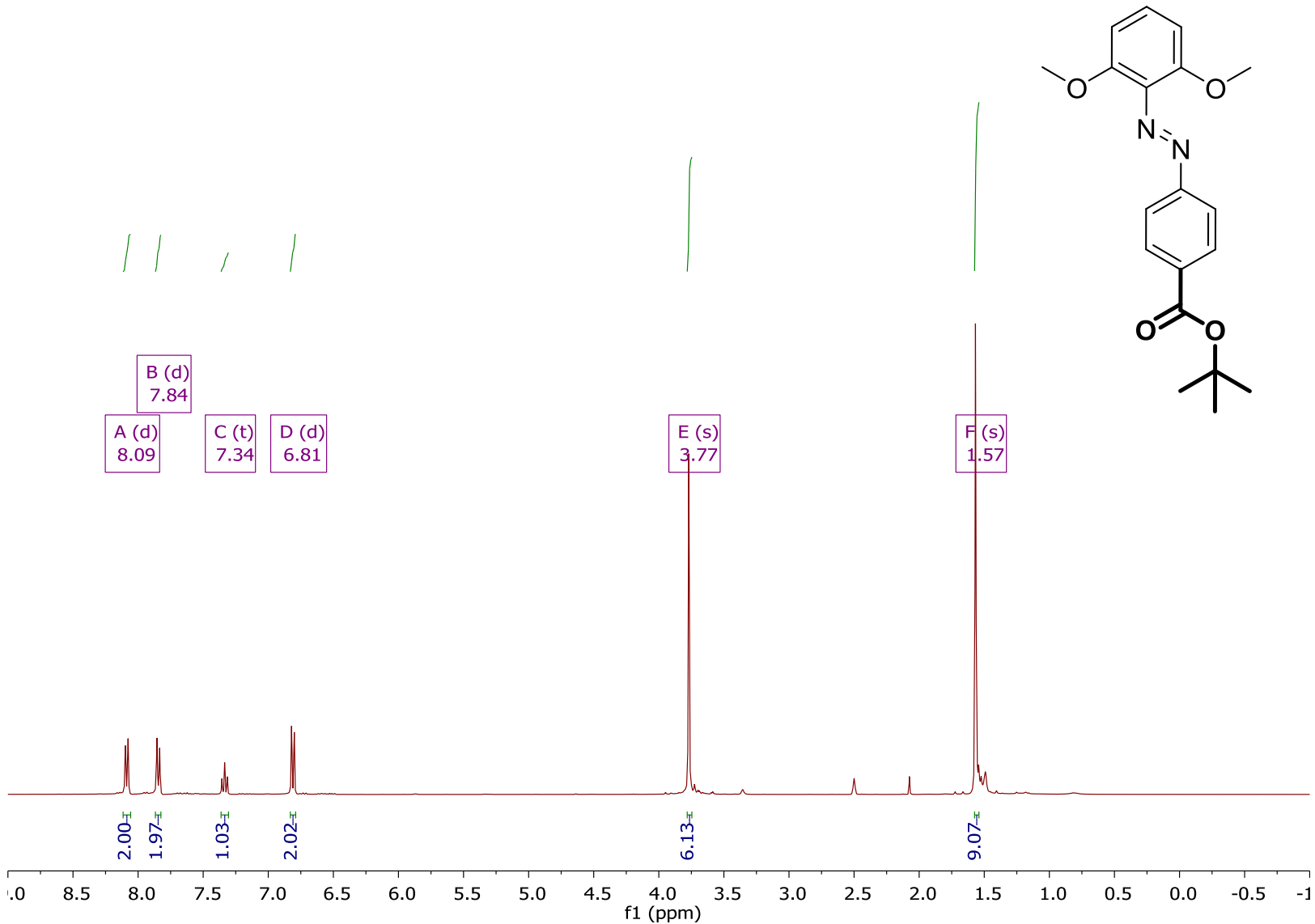




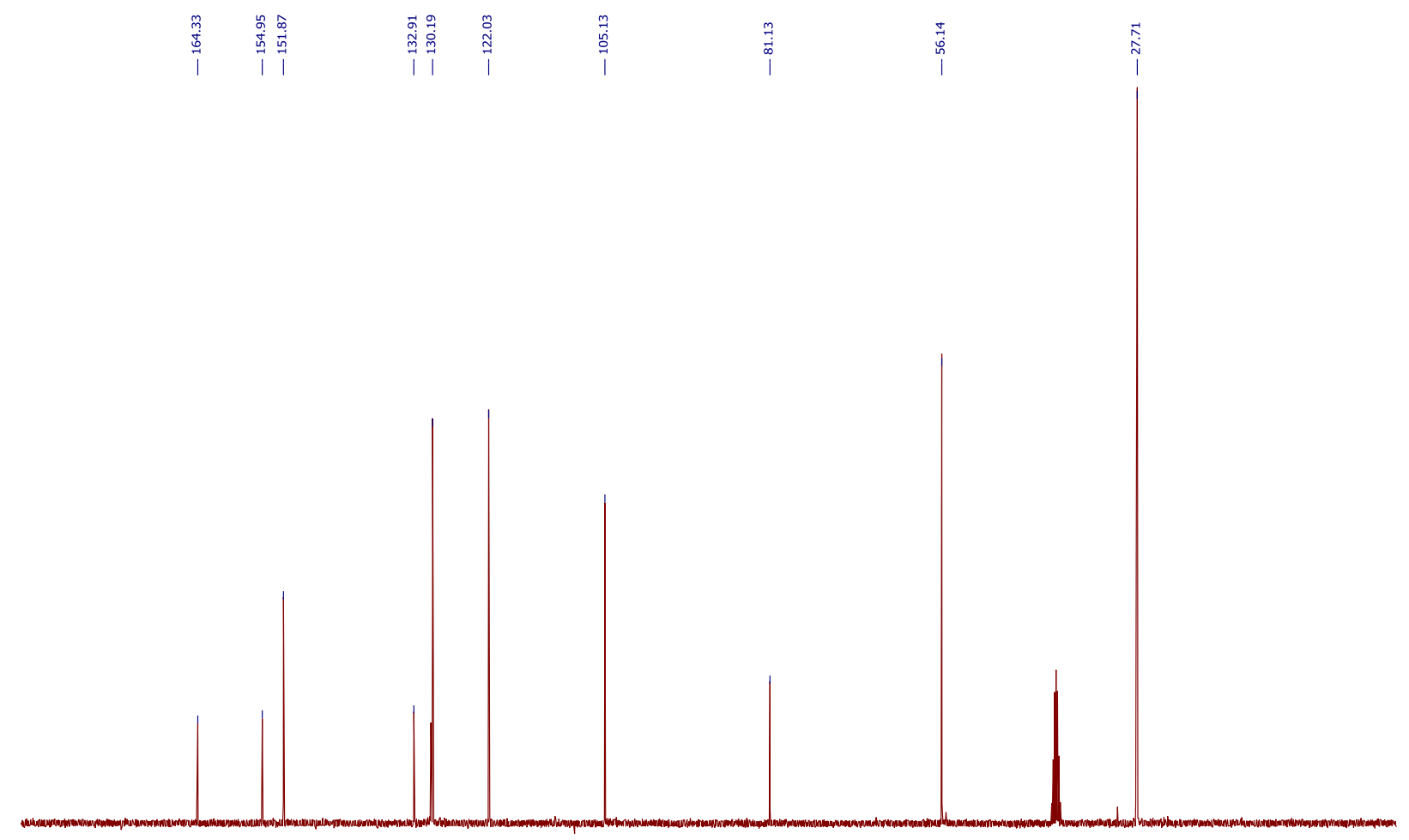

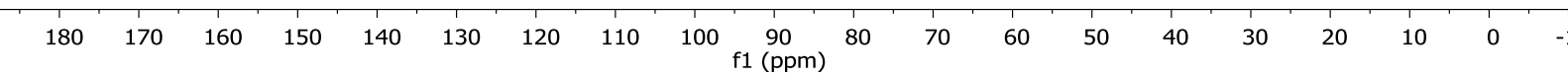

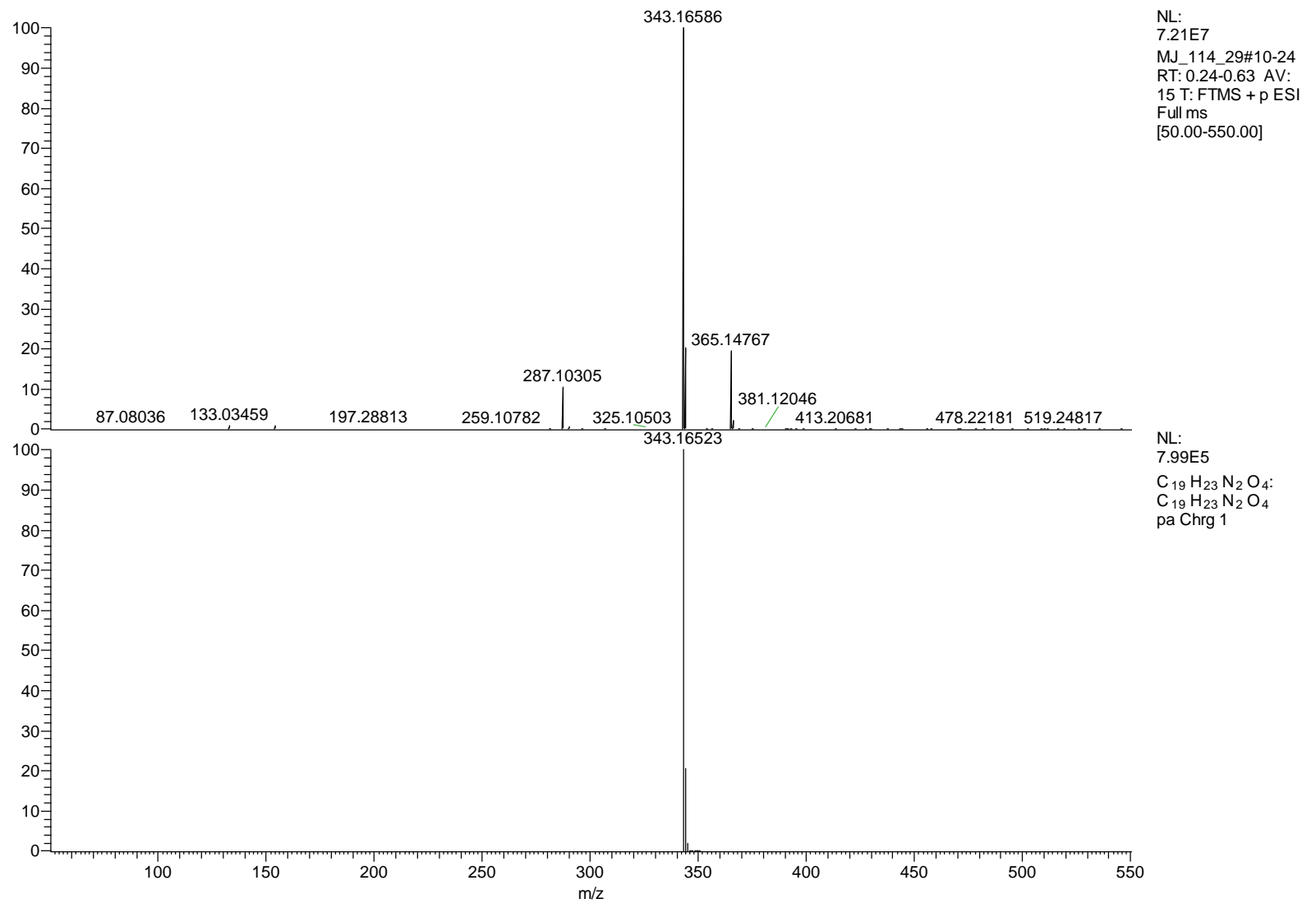


3g:
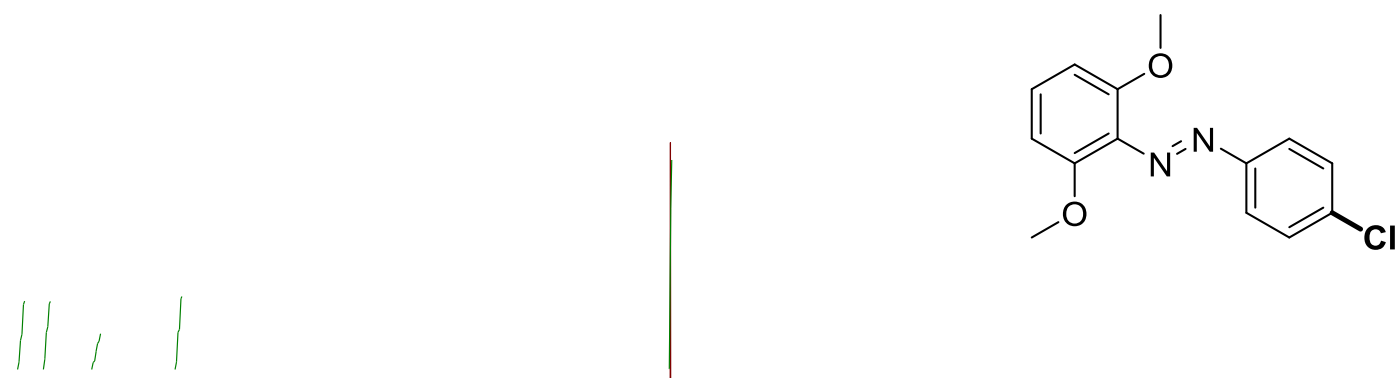

$$
\begin{aligned}
& \begin{array}{|l|}
\hline \text { B (d) } \\
7.61 \\
\hline A(d) \\
\hline
\end{array} \\
& \begin{array}{|l|l|l|l|l|}
\hline \text { A (d) } & \text { C (t) } & \text { D (d) } \\
\hline
\end{array} \\
& \begin{array}{llll}
\hline 7.77 & 7.31 & 6.79 \\
\hline
\end{array}
\end{aligned}
$$

\begin{tabular}{|c|c|c|c|}
\hline 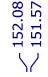 & 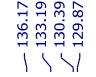 & 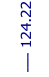 & $\begin{array}{l}\text { No } \\
\text { ஸे } \\
\stackrel{0}{0} \\
\mid\end{array}$ \\
\hline
\end{tabular}
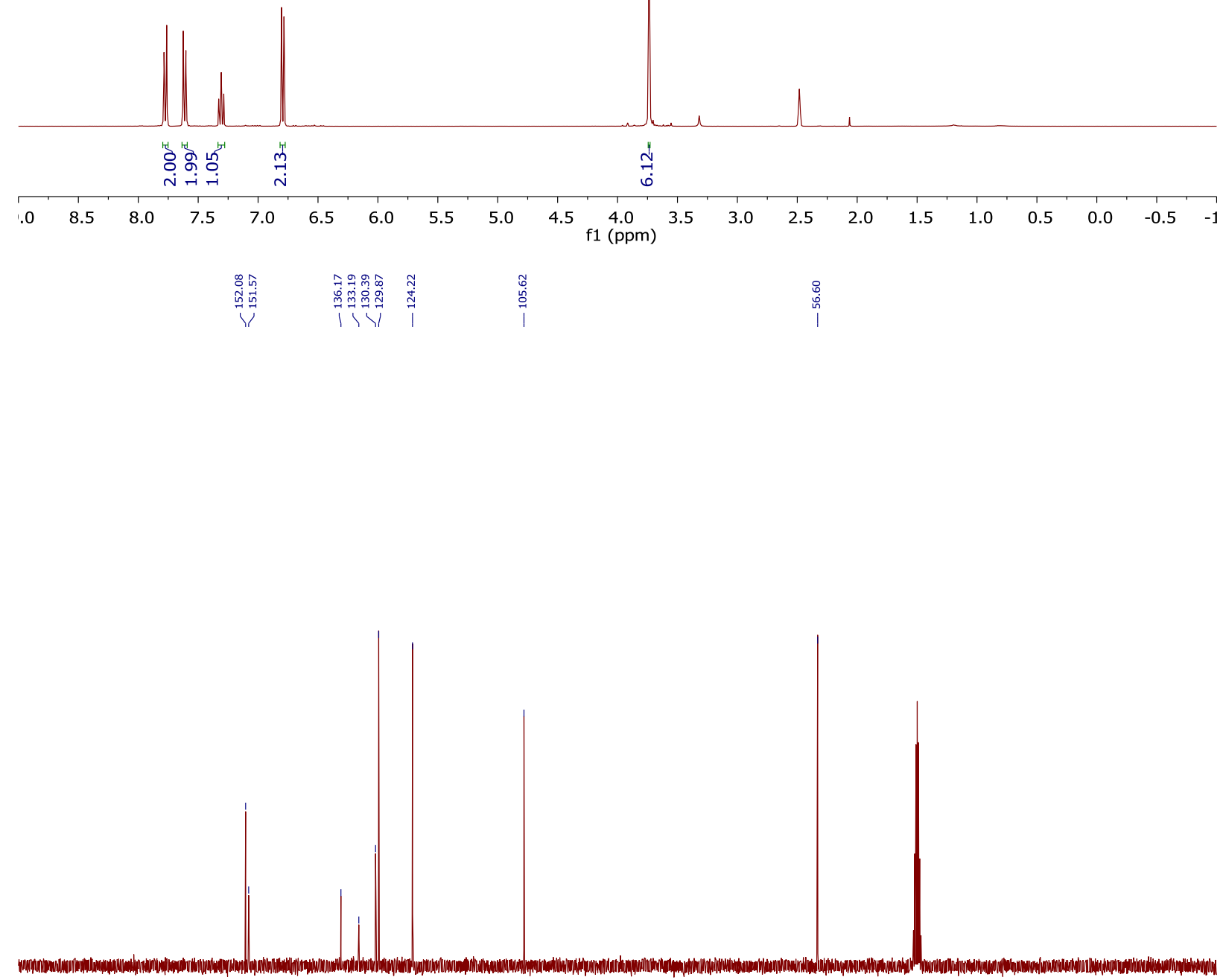


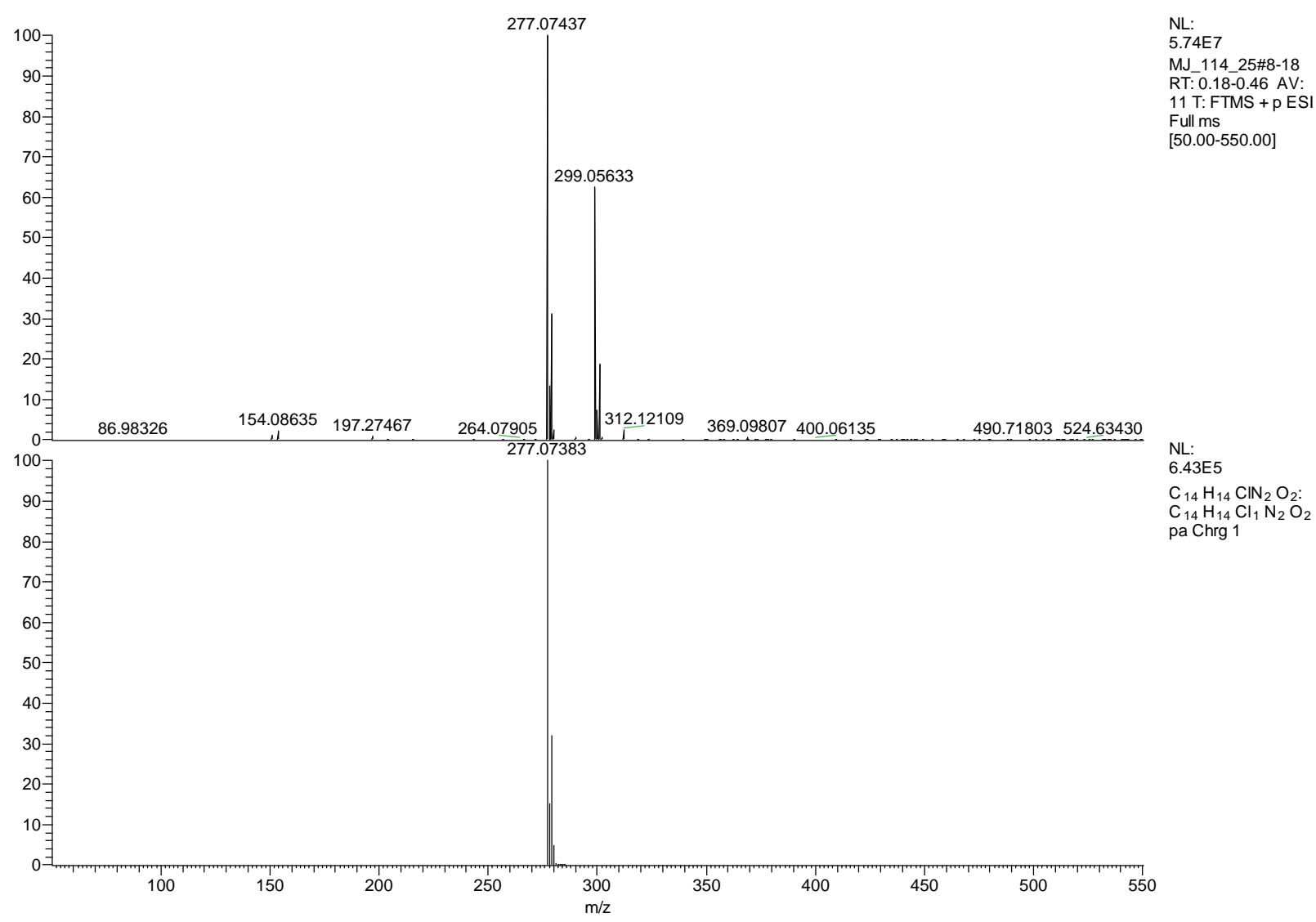

3h:

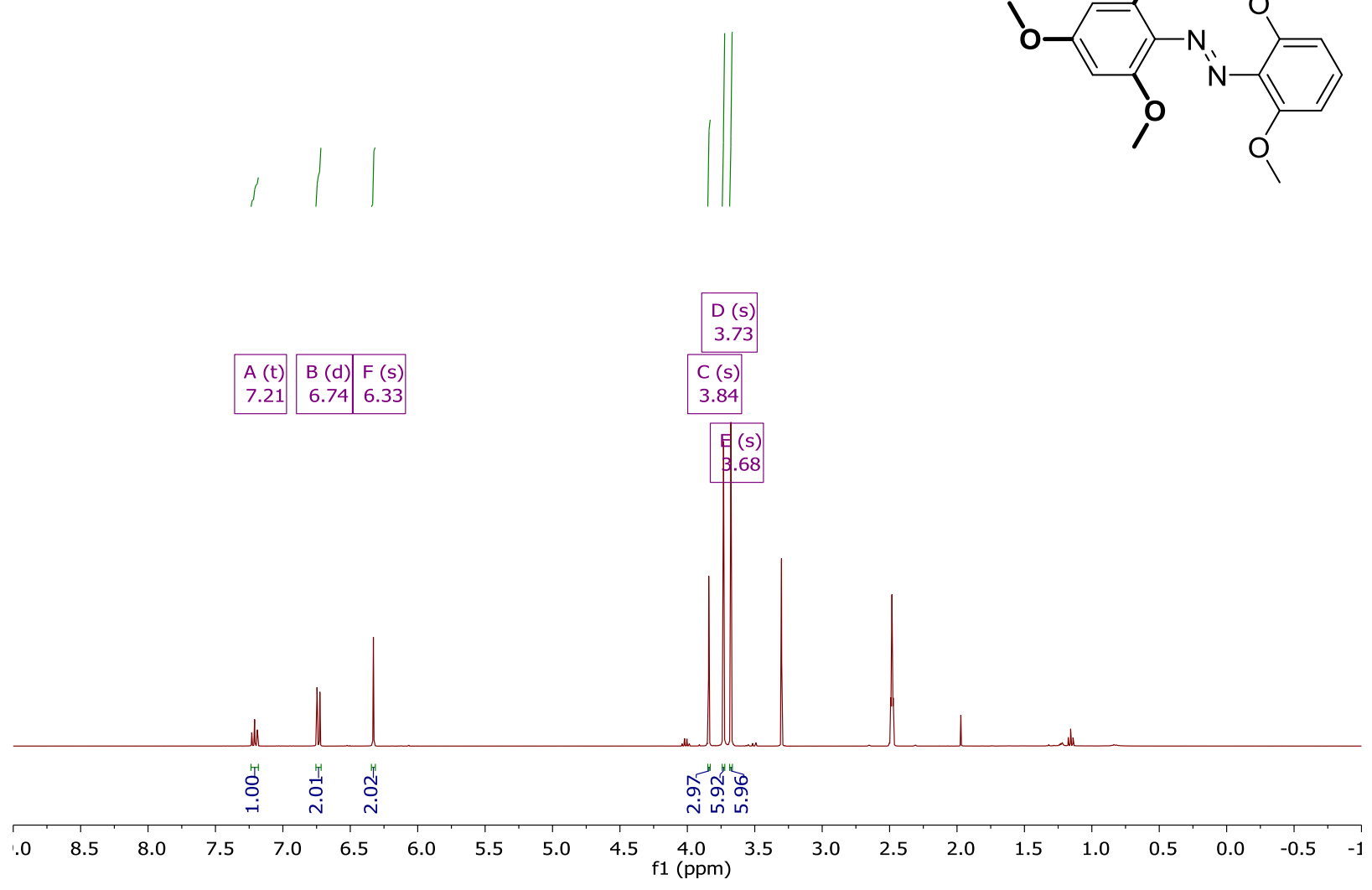




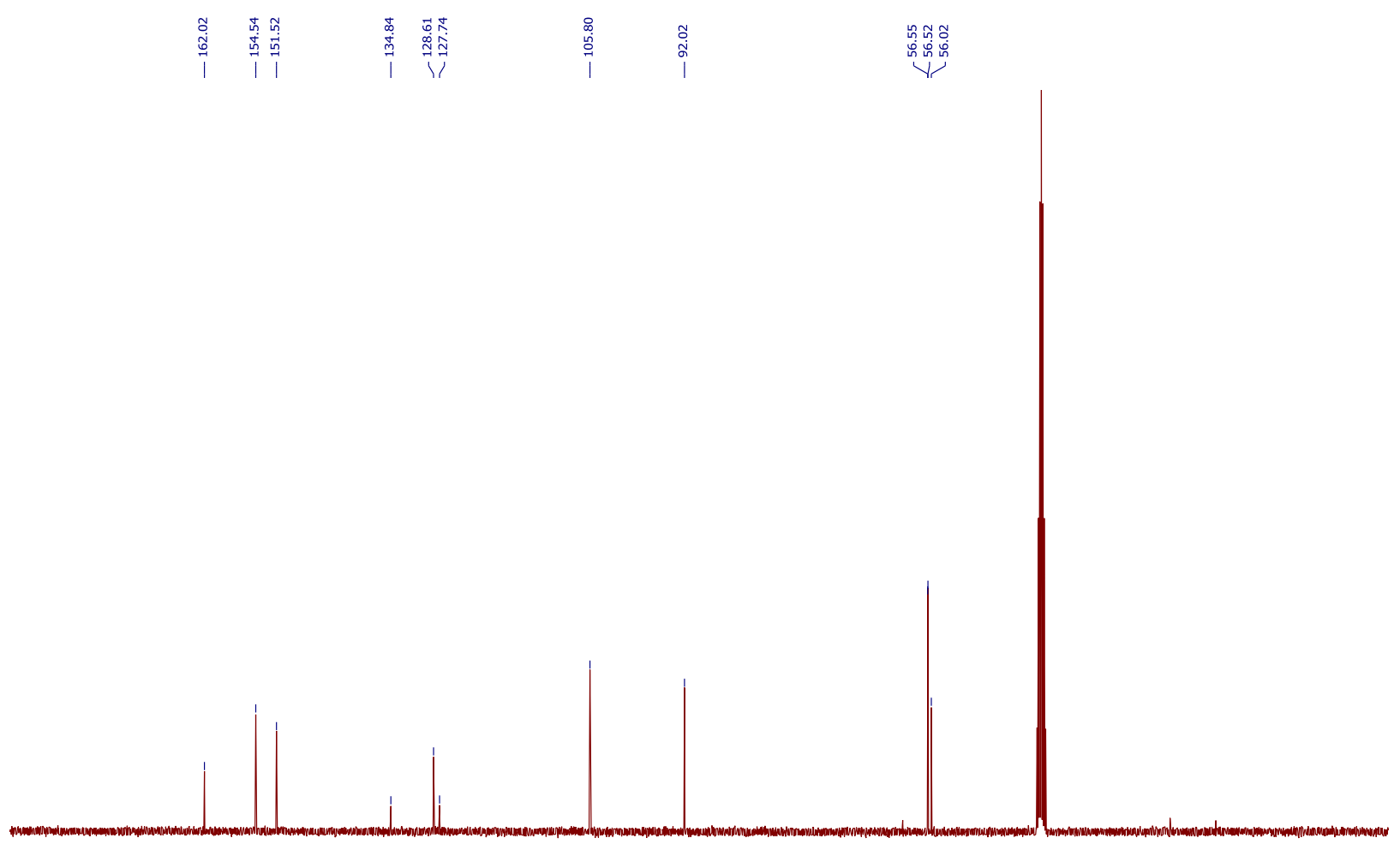

\begin{tabular}{lllllllllllllllllllll}
\hline 90 & 180 & 170 & 160 & 150 & 140 & 130 & 120 & 110 & 100 & $\begin{array}{c}90 \\
\mathrm{f} 1(\mathrm{ppm})\end{array}$ & 80 & 70 & 60 & 50 & 40 & 30 & 20 & 10 & 0 & -1
\end{tabular}

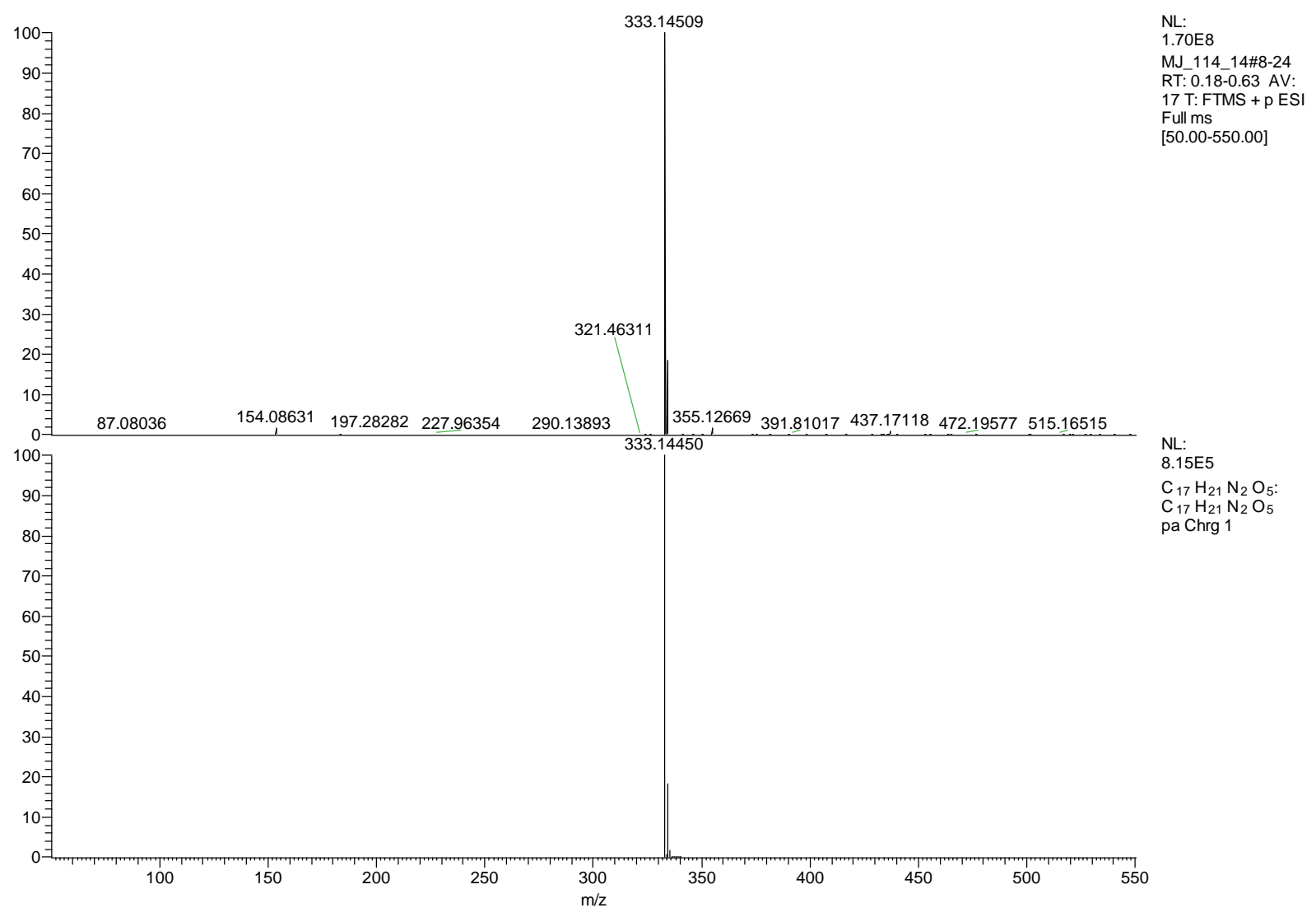


3i:

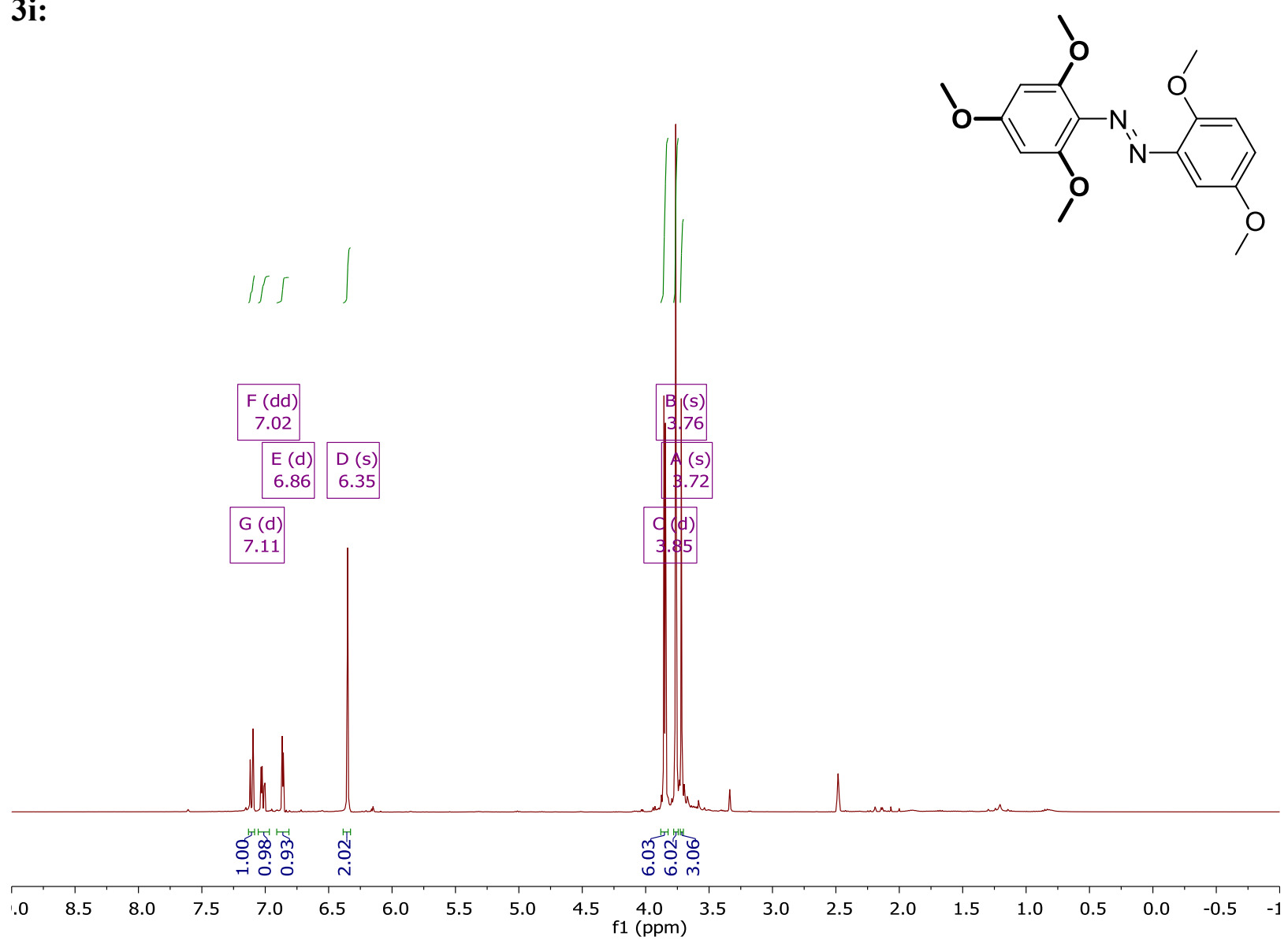

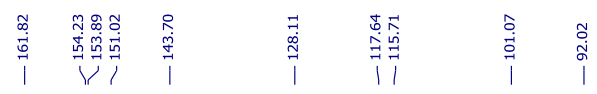

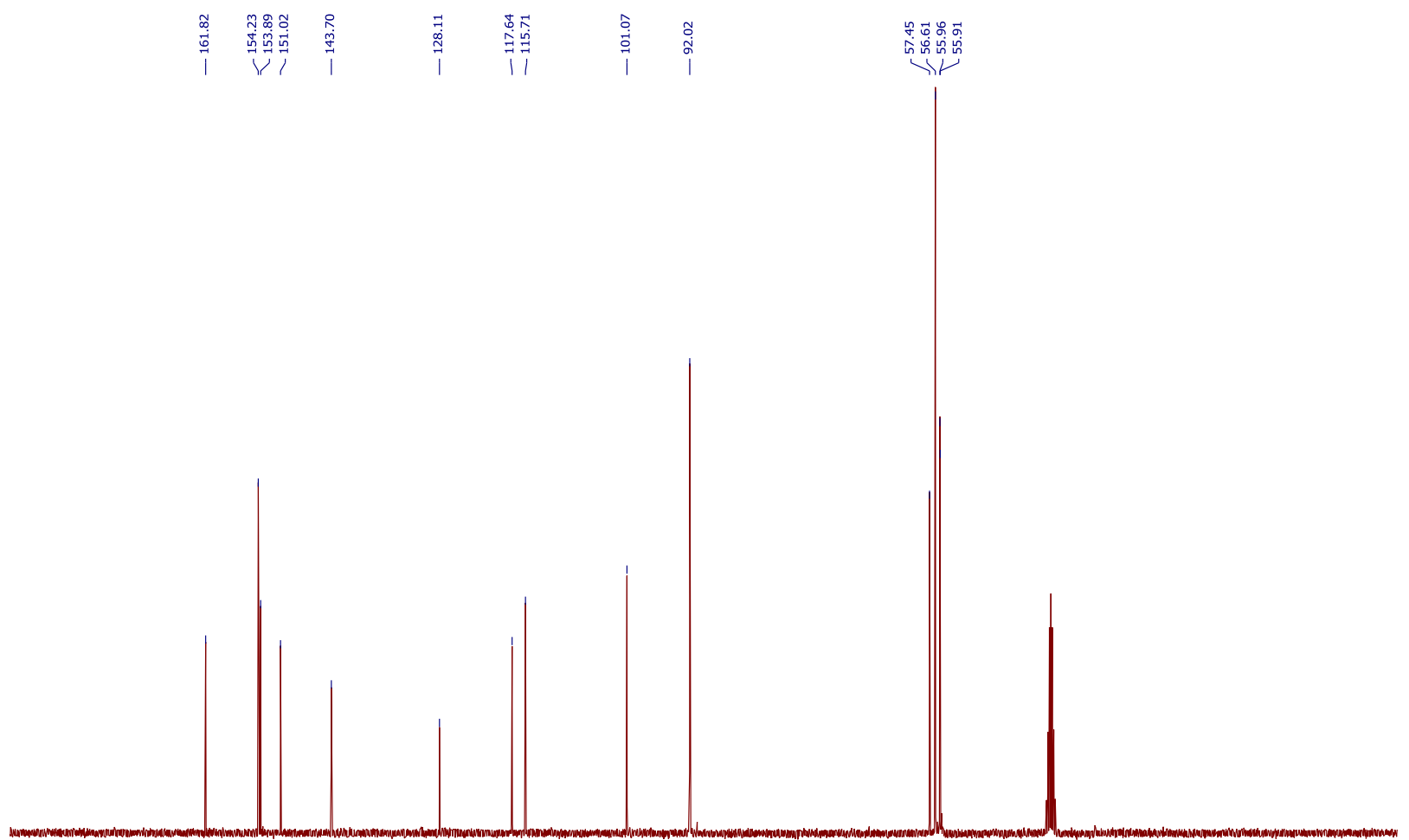

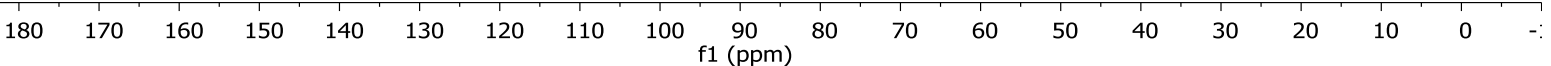




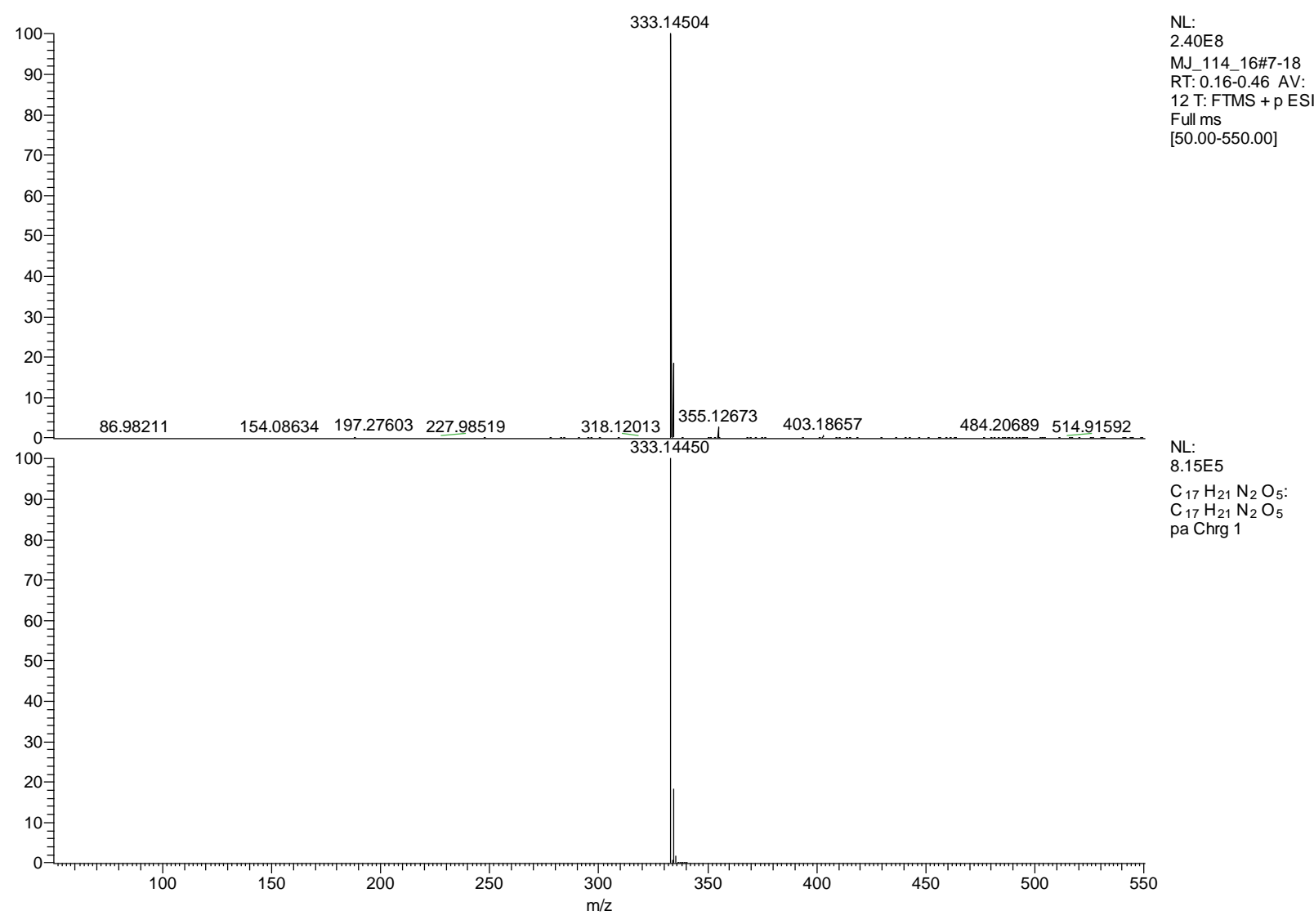

3j: After thermal adaption, no full conversion to the trans isomer was observed which became apparent in the ${ }^{1} \mathrm{H}-\mathrm{NMR}$ and ${ }^{13} \mathrm{C}$-NMR spectrum showing small quantities of the cis isomer.

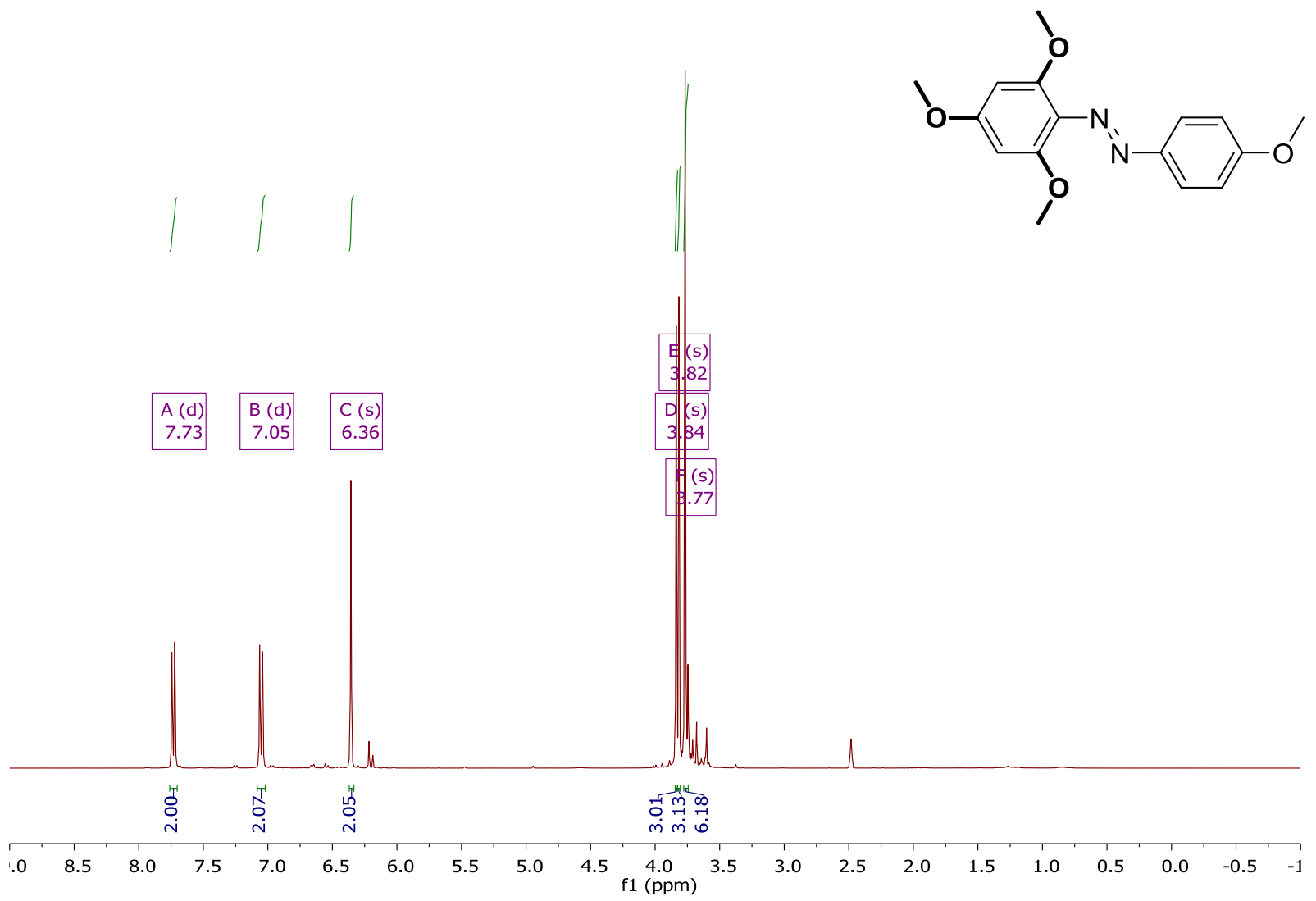




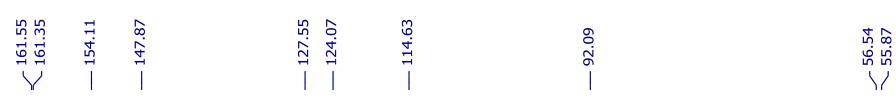

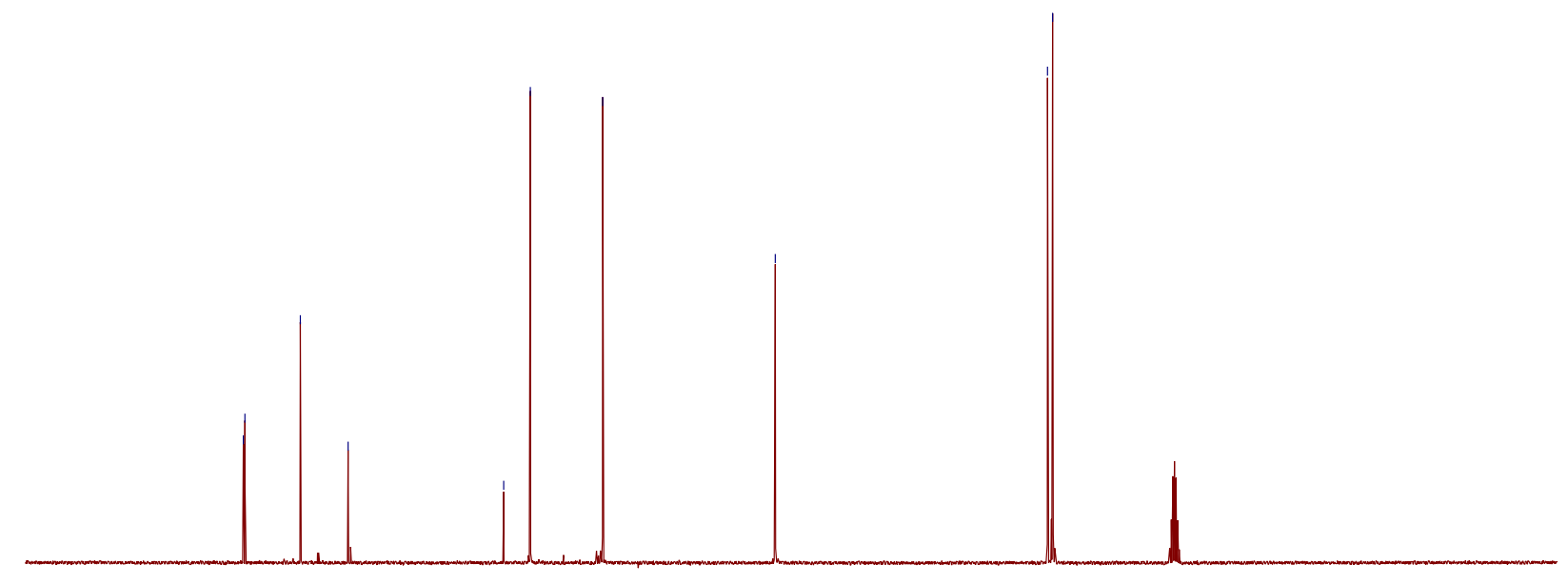

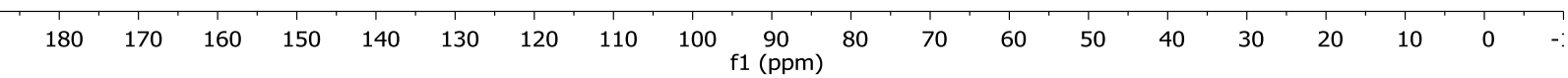

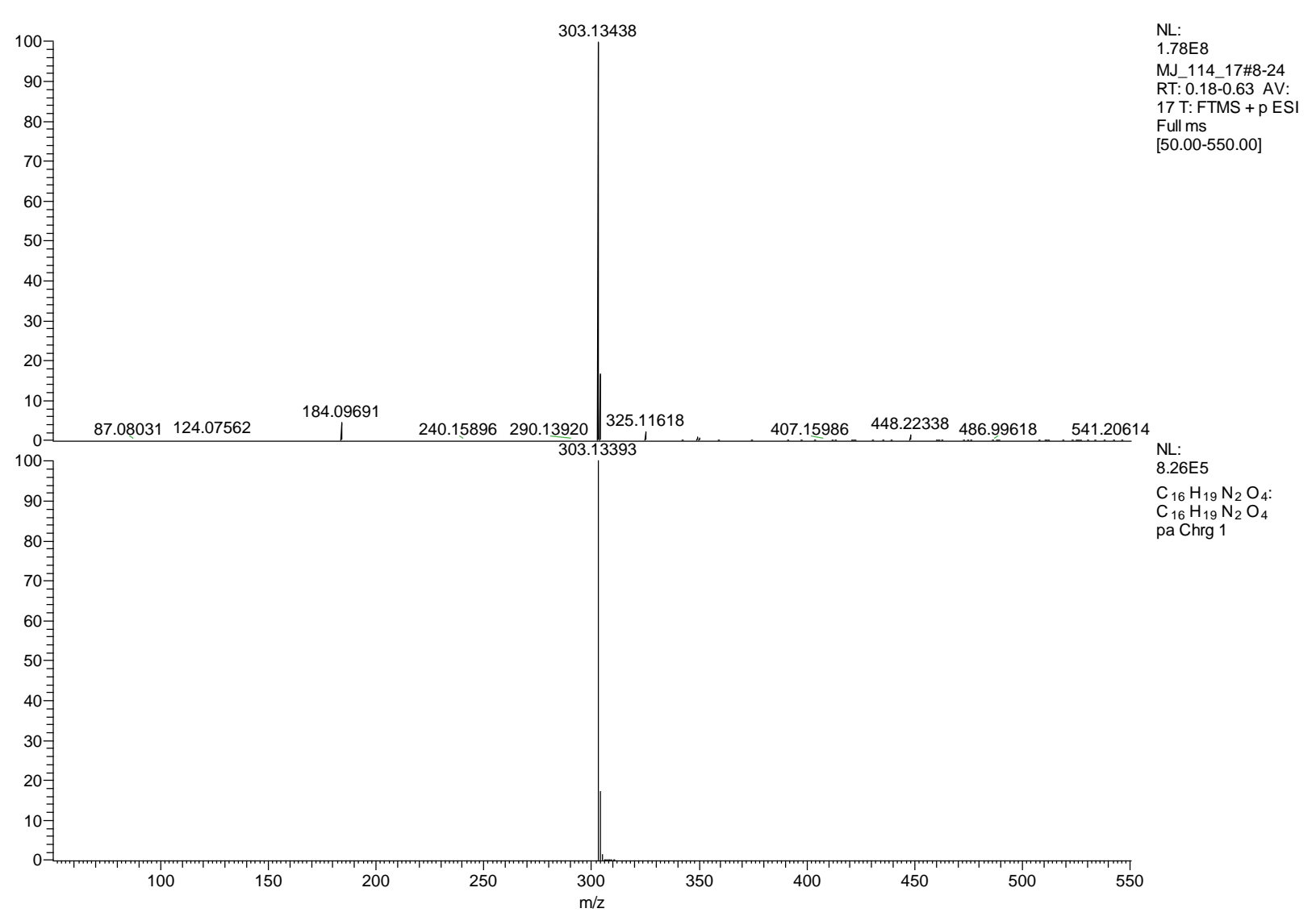


3k:

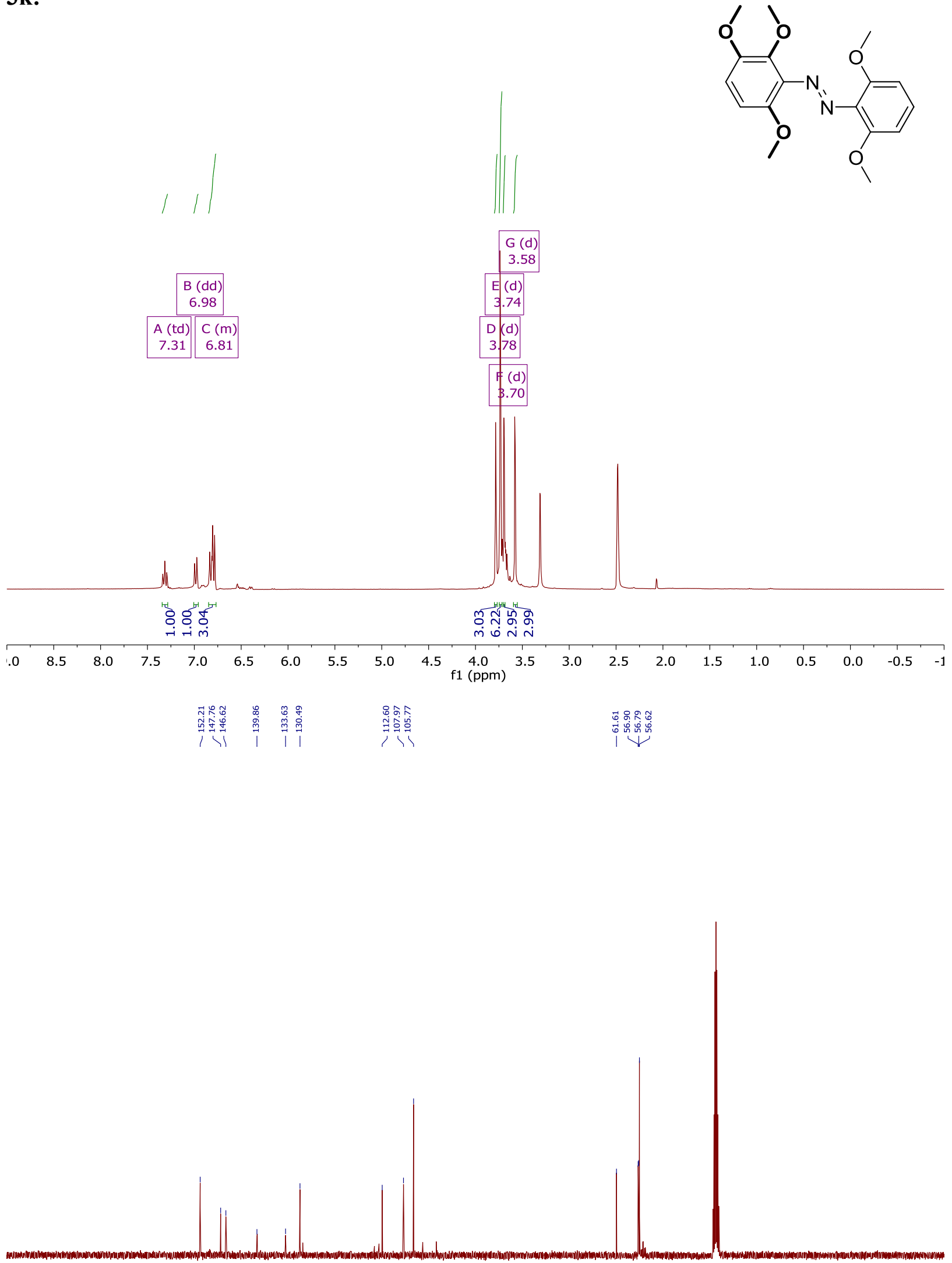

$\begin{array}{lllllllllllllllllllllll}190 & 180 & 170 & 160 & 150 & 140 & 130 & 120 & 110 & 100 & 90 & 80 & 70 & 60 & 50 & 40 & 30 & 20 & 10 & 0 & 10\end{array}$ 


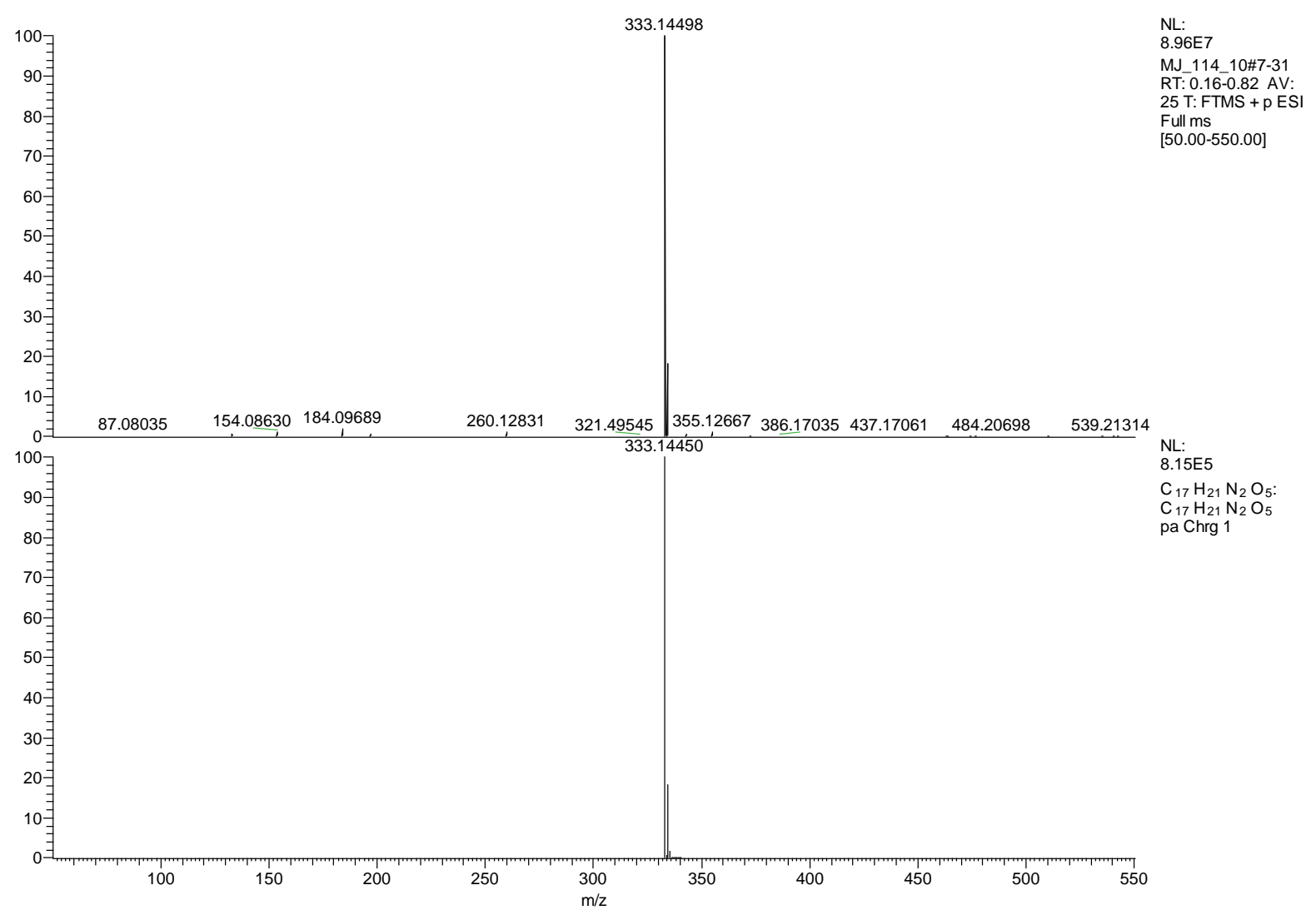

31:
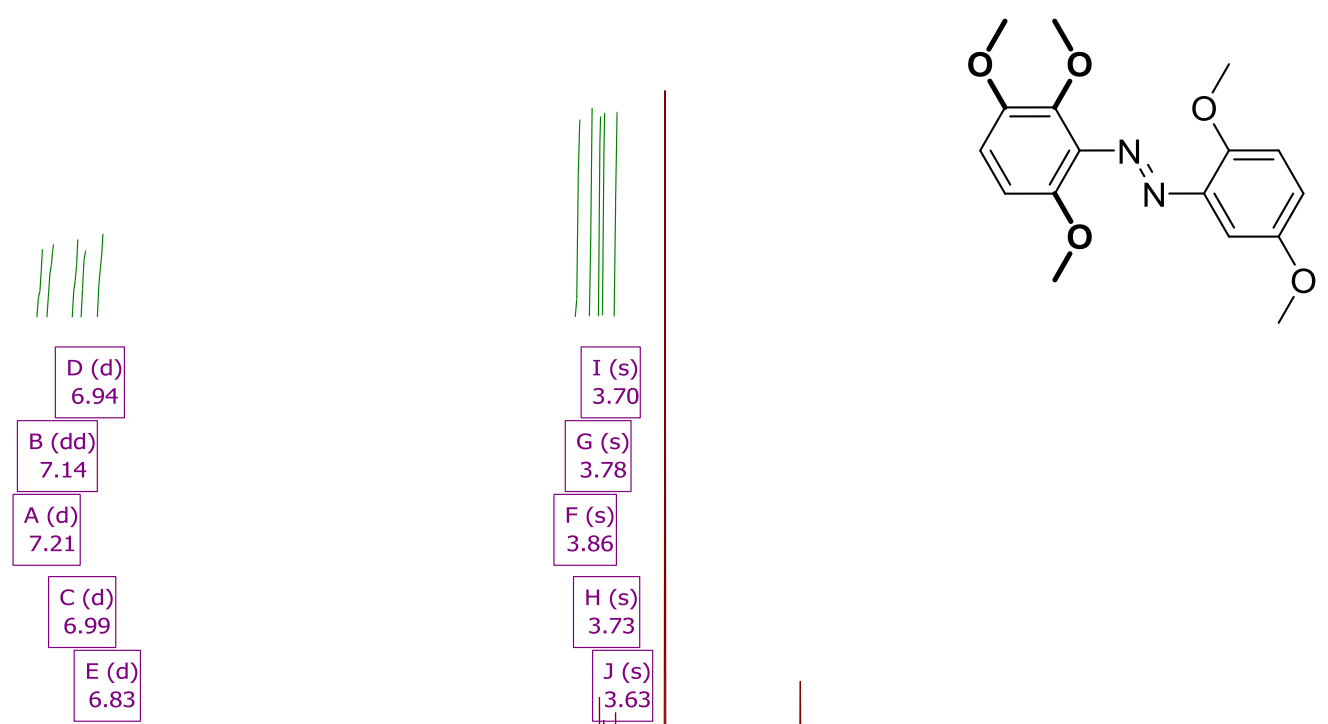

Wh the

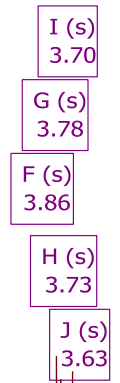

웅

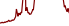

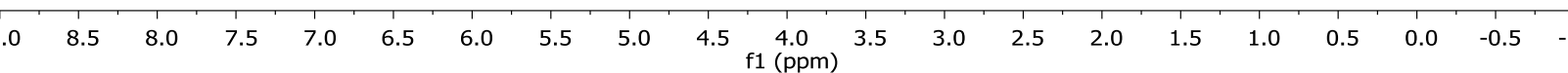




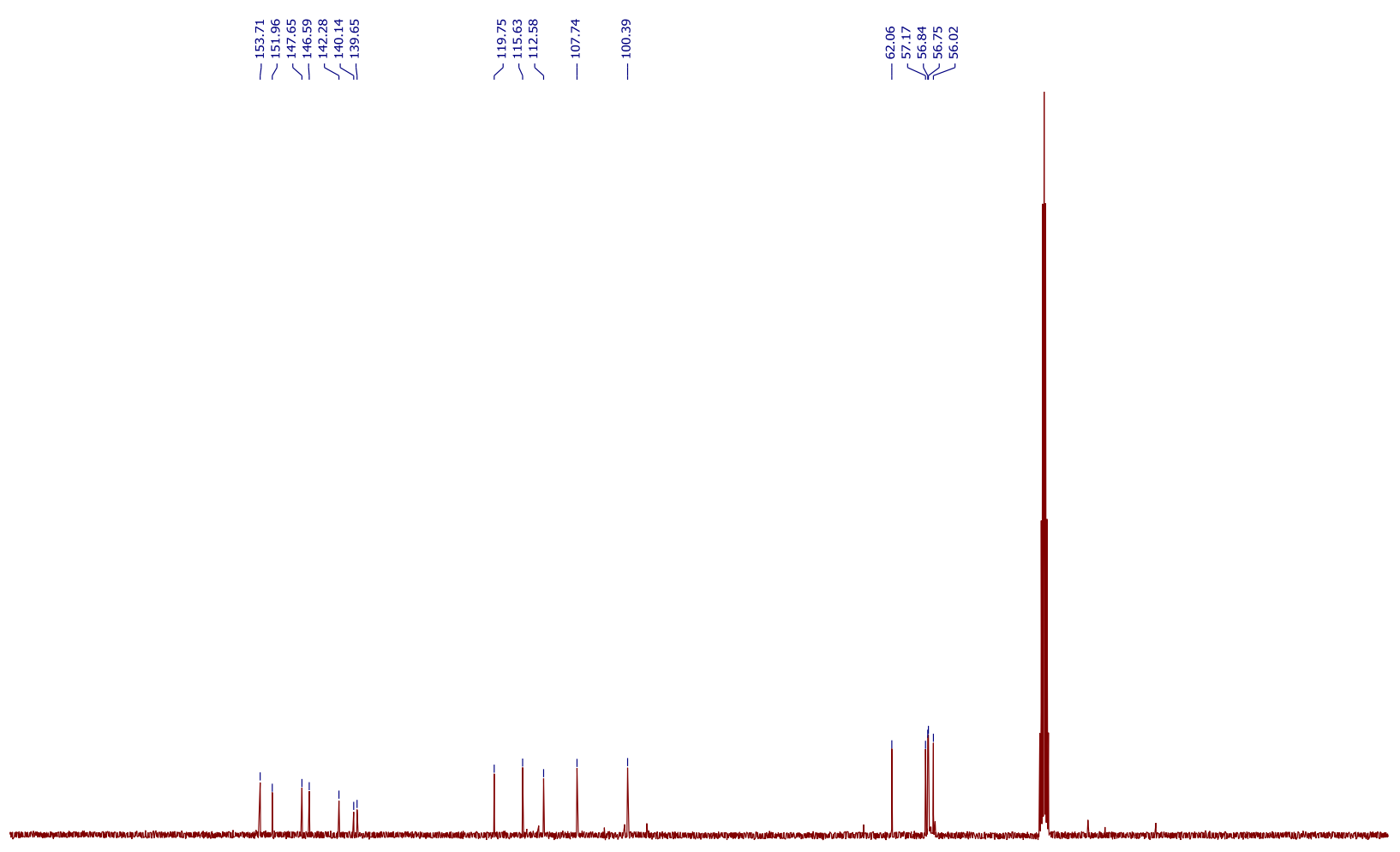

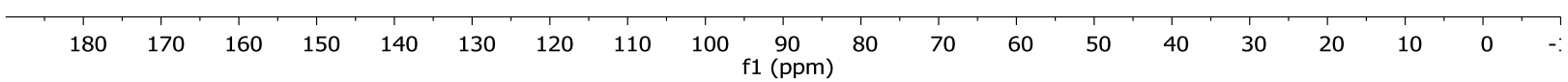

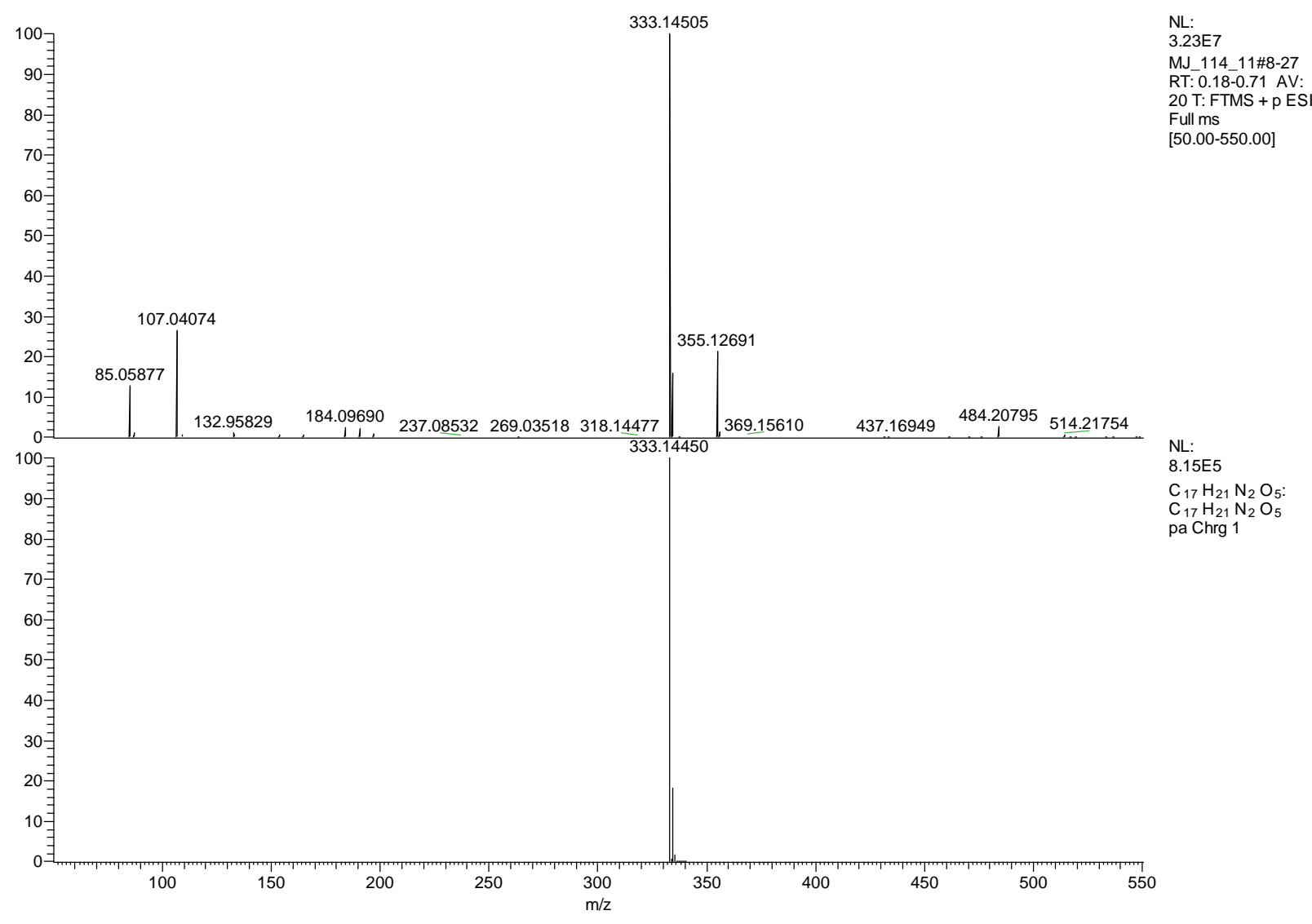


3m:

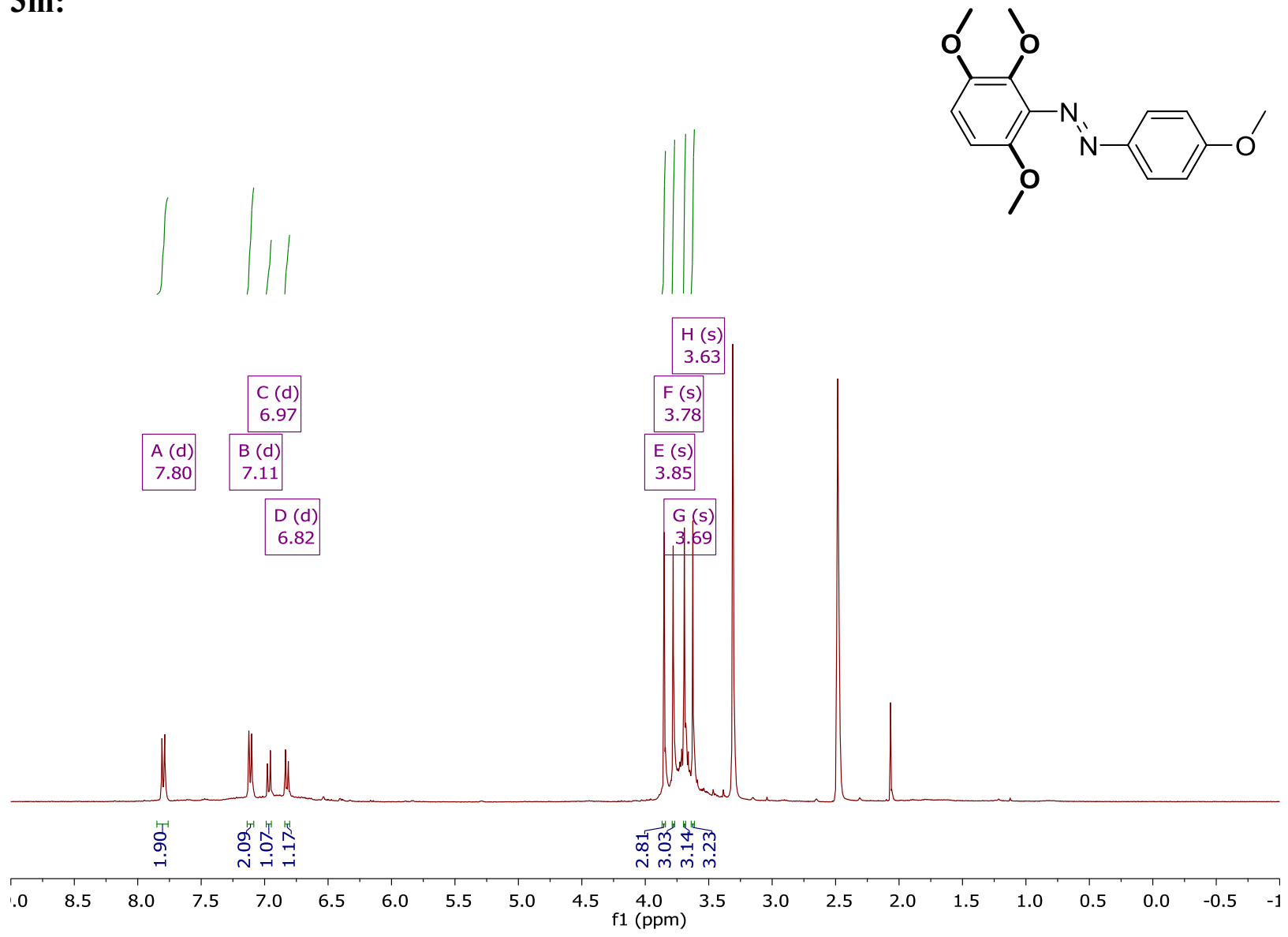

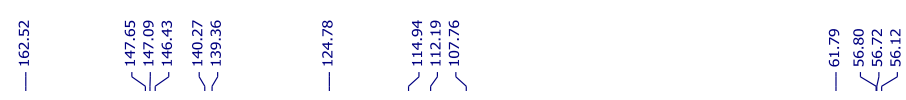

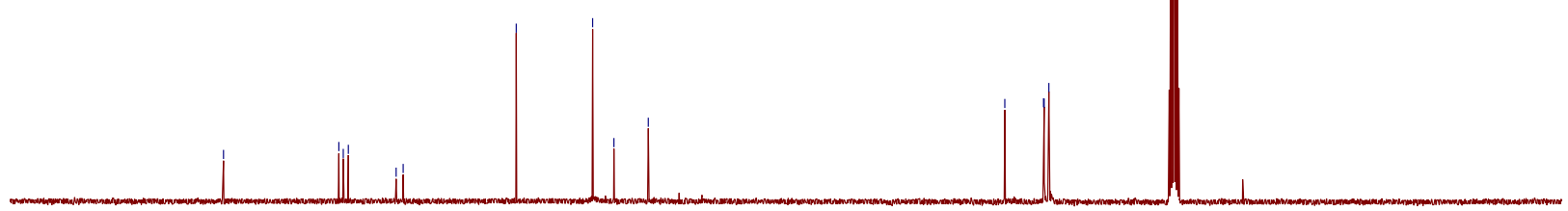

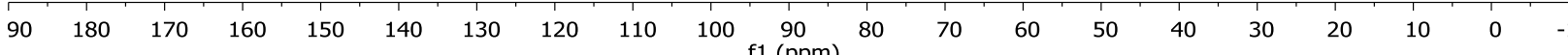




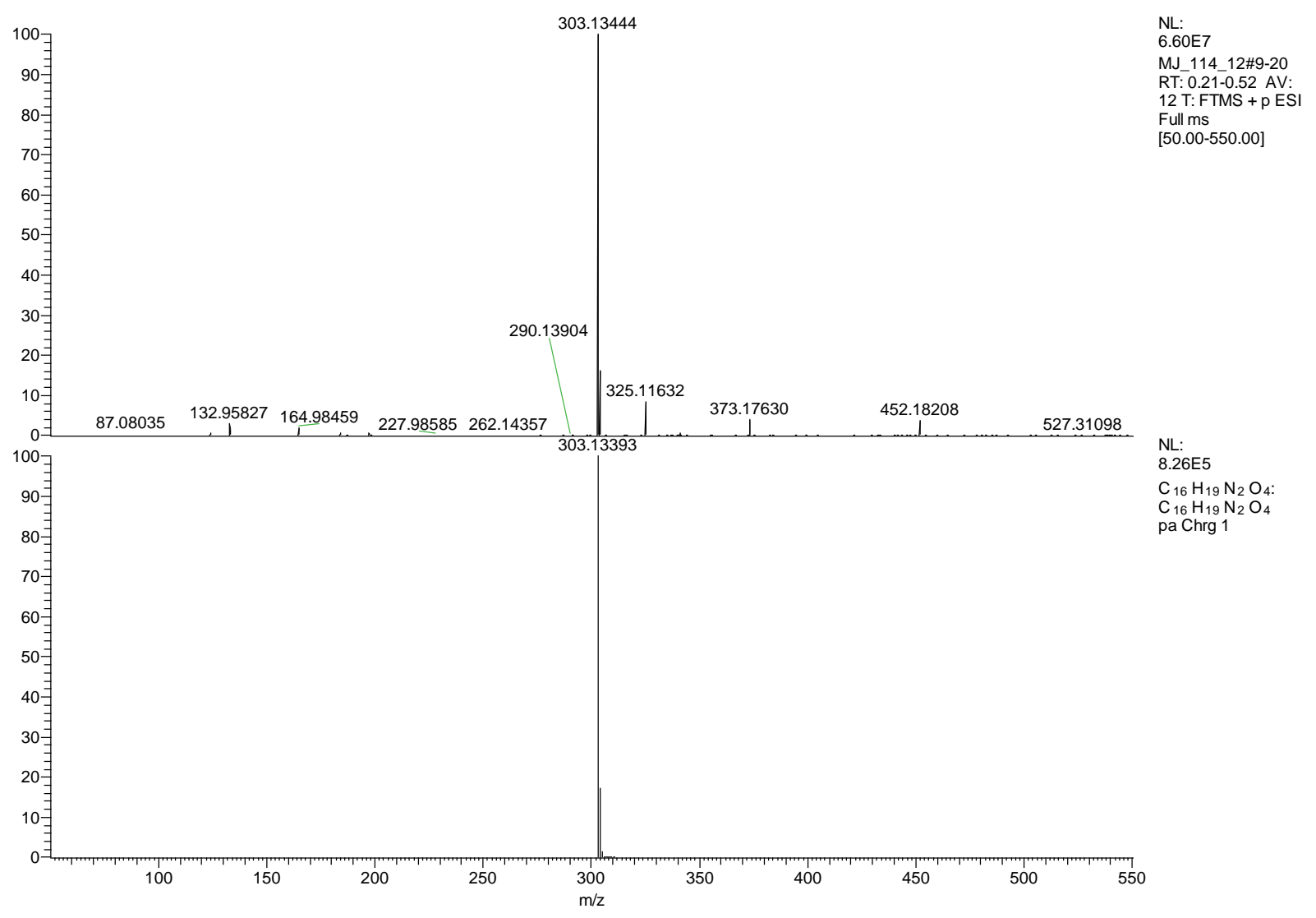

3n:
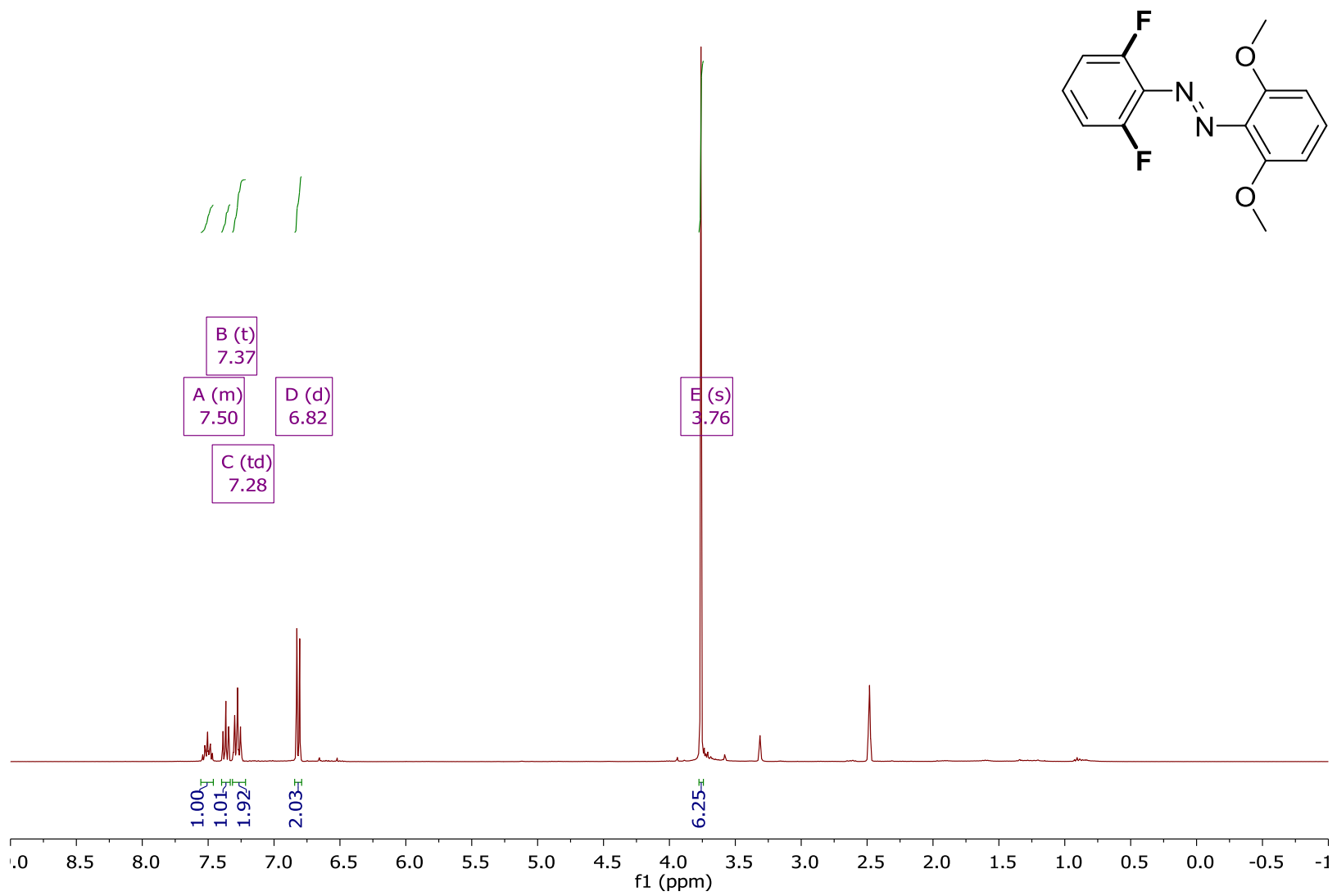


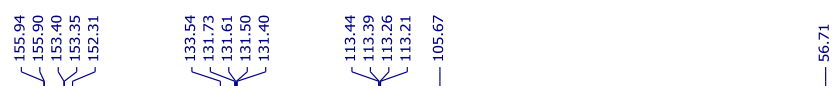
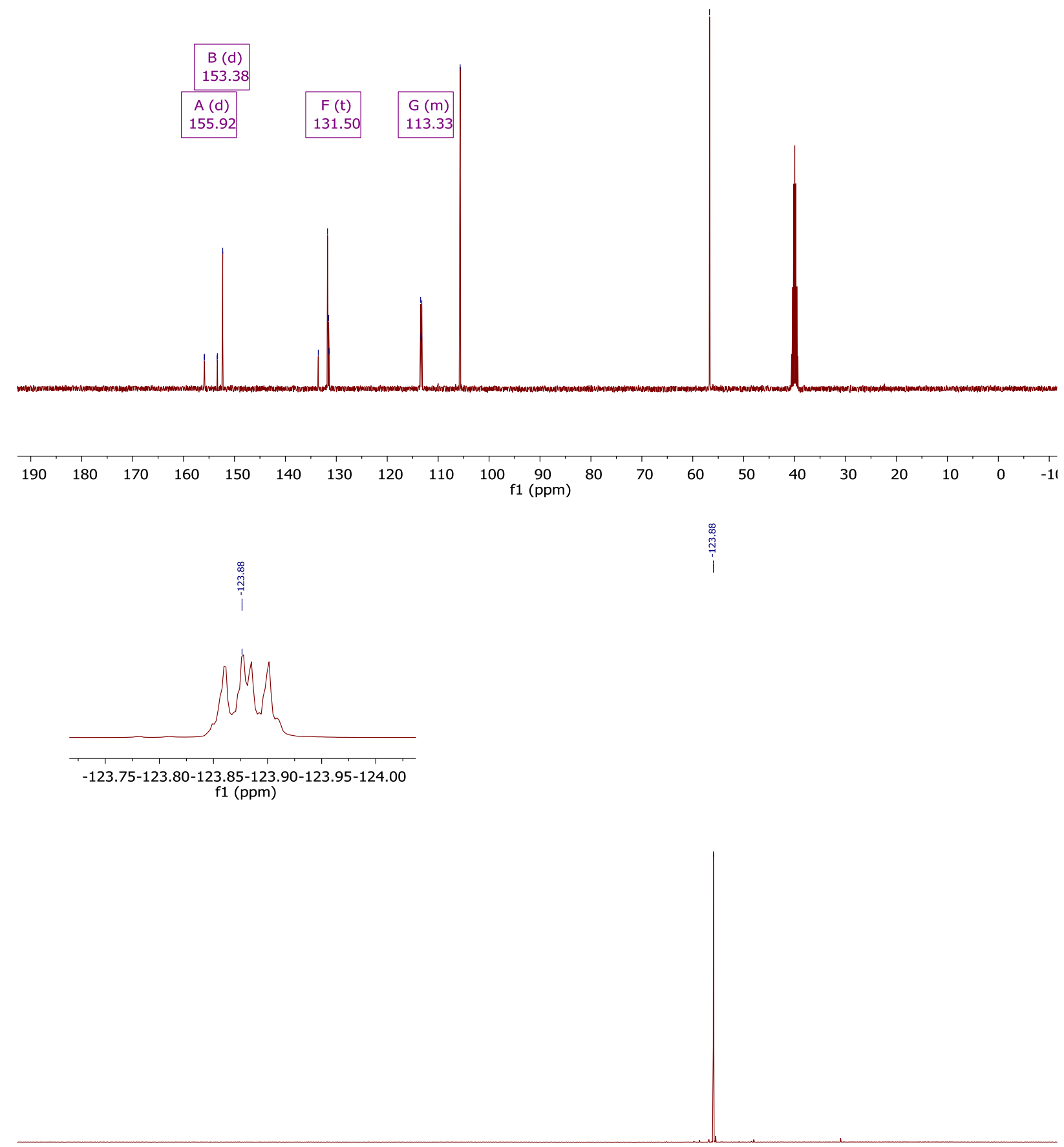

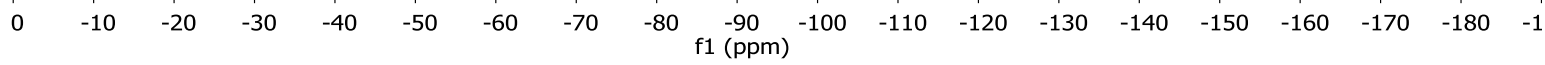




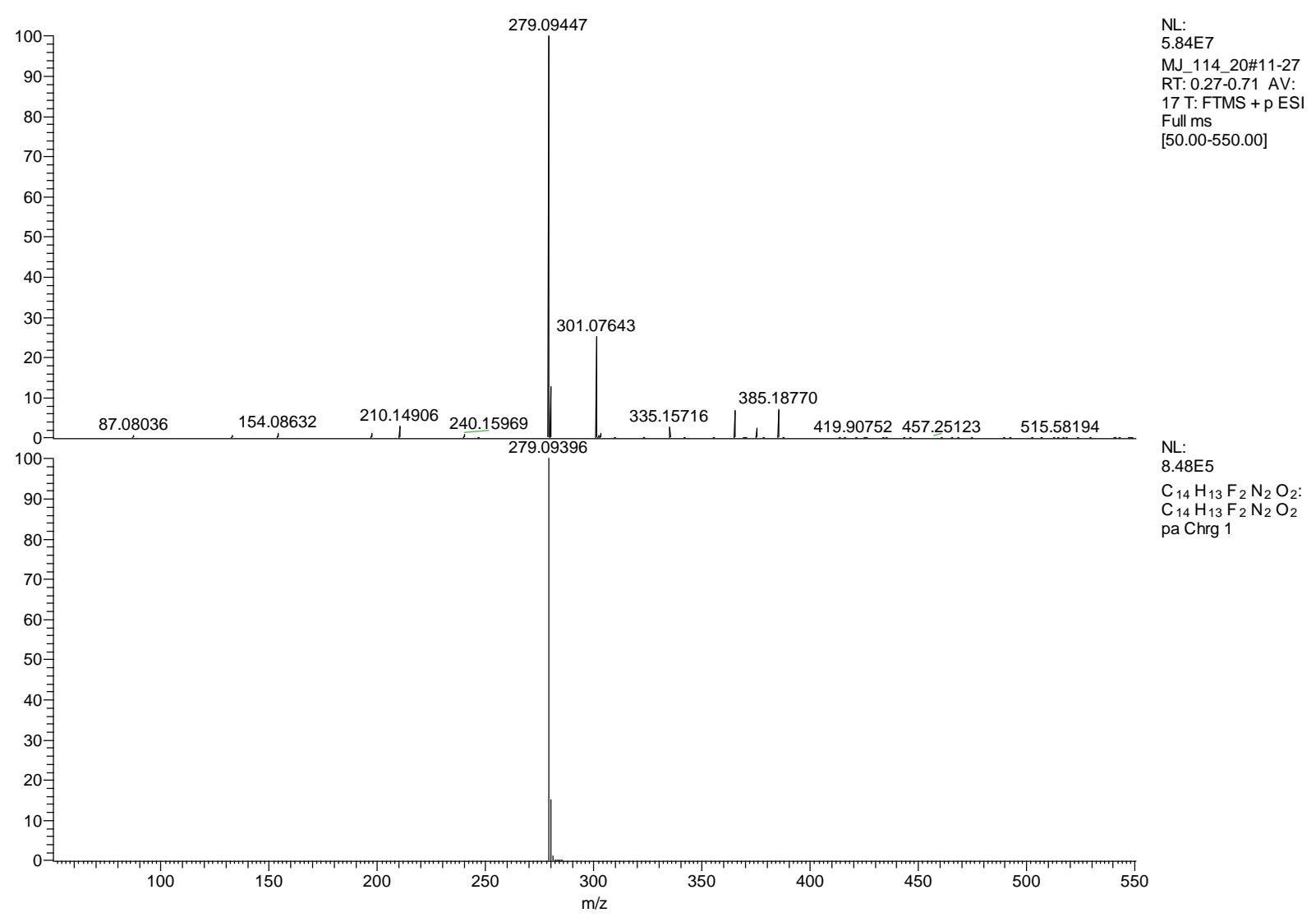

3o: After thermal adaption, no full conversion to the trans isomer was observed which became apparent in the ${ }^{1} \mathrm{H}-\mathrm{NMR}$ and ${ }^{13} \mathrm{C}-\mathrm{NMR}$ spectrum showing small quantities of the cis isomer.
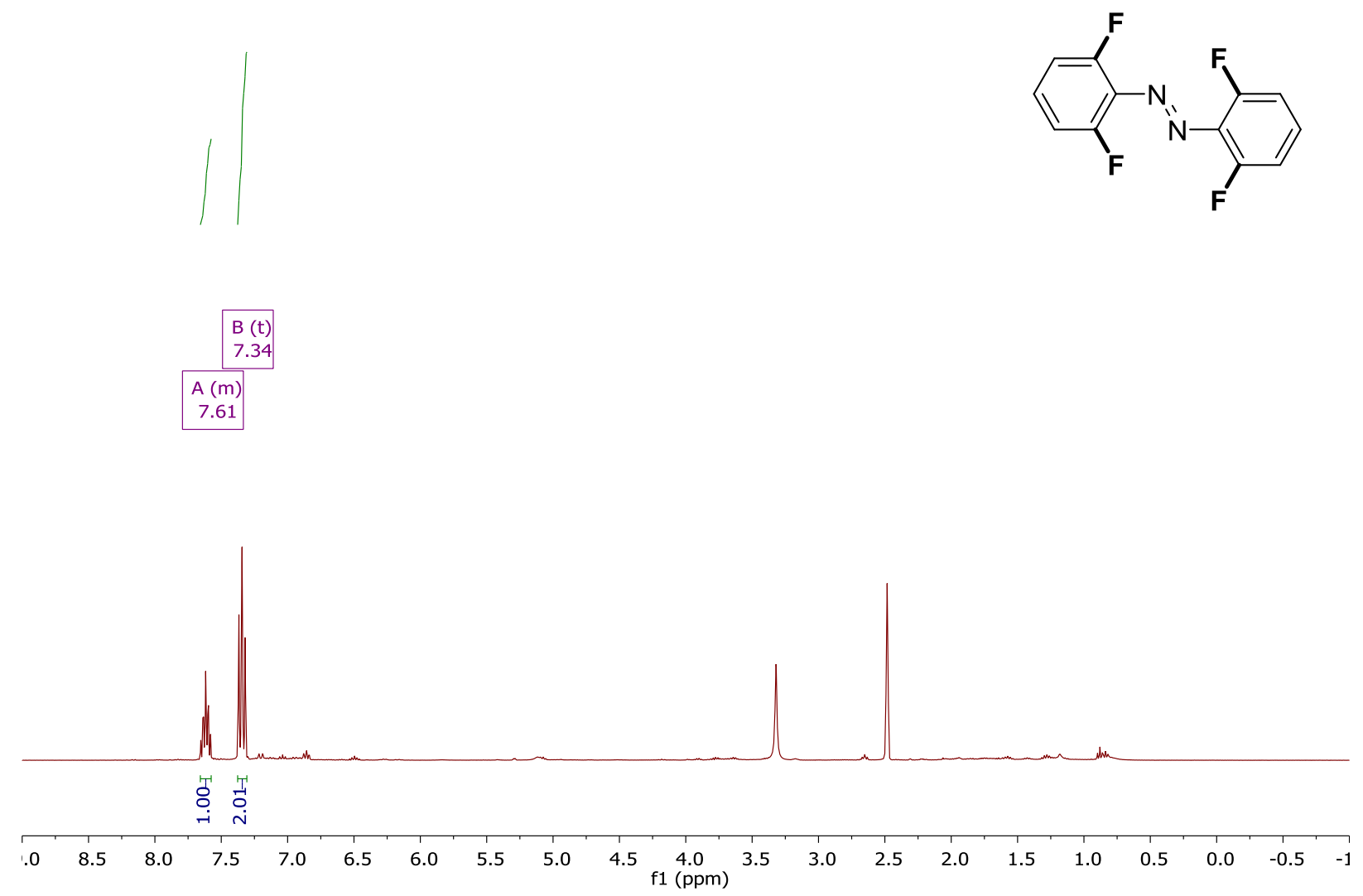
V| v

\begin{tabular}{|c|c|c|}
\hline $\begin{array}{r}B(d) \\
153.7\end{array}$ & & \\
\hline $\begin{array}{c}A(d) \\
156.35\end{array}$ & $\begin{array}{c}C(t) \\
133.62\end{array}$ & $\begin{array}{c}D(d d) \\
113.65\end{array}$ \\
\hline
\end{tabular}

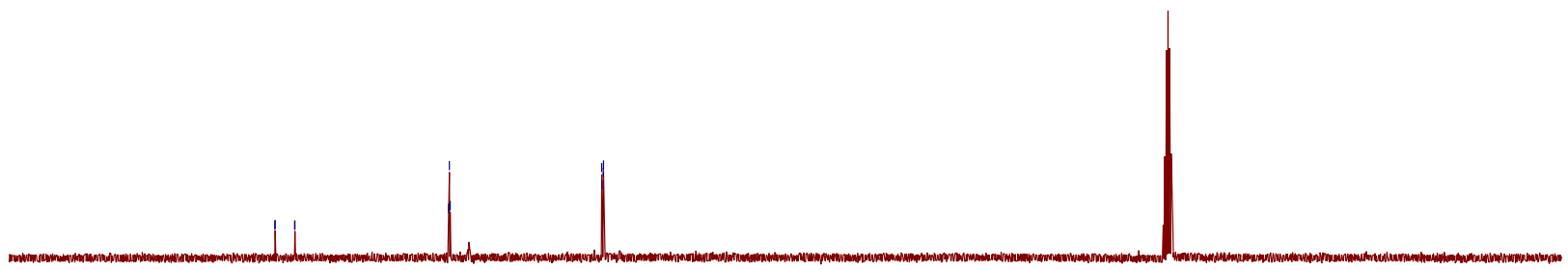

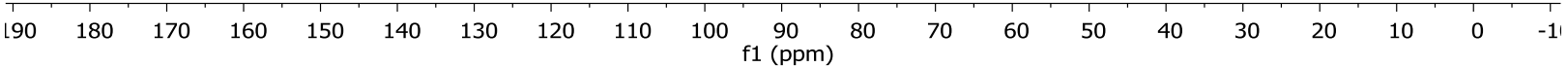

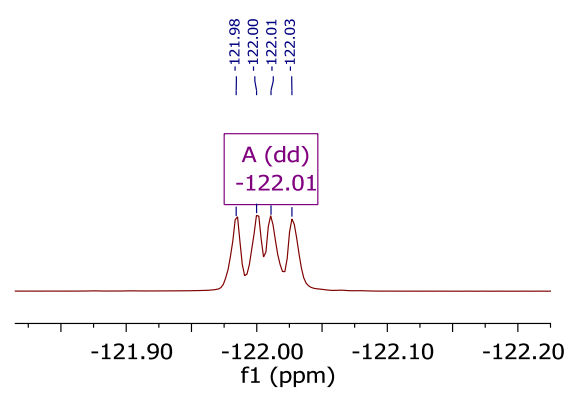

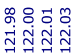

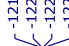

A (dd)

$-122.01$

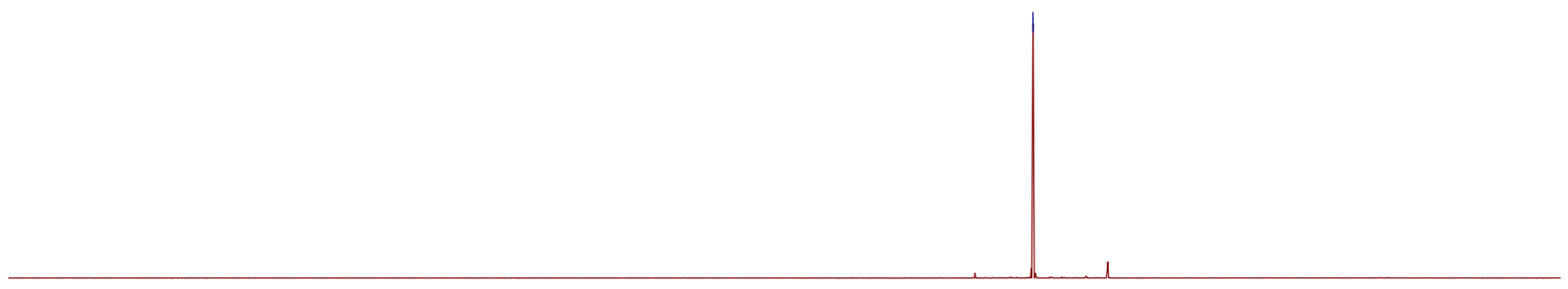

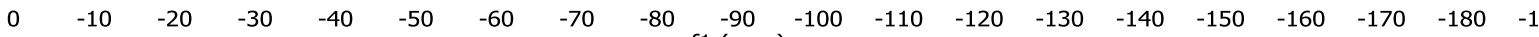
f1 (ppm) 


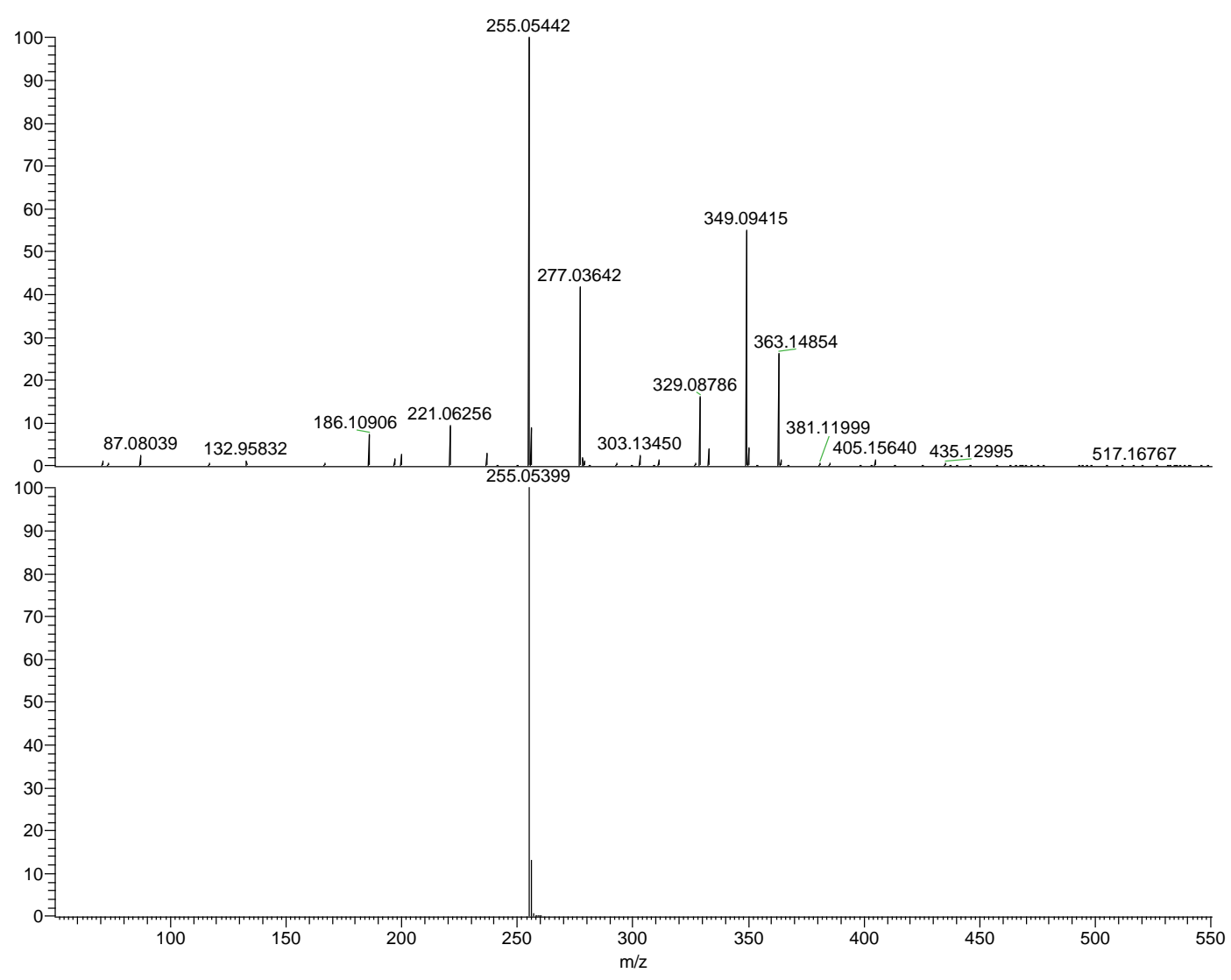

NL:

MJ 114 21\#7-22

RT: $0.16-0.57 \mathrm{AV}$ :

$16 \mathrm{~T}: \mathrm{FTMS}+\mathrm{p}$ ESI

Full ms
$[50.00-550.00]$
$\mathrm{NL}:$
$8.72 \mathrm{E} 5$

$\mathrm{C}_{12} \mathrm{H}_{7} \mathrm{~F}_{4} \mathrm{~N}_{2}$ :

$\mathrm{C}_{12} \mathrm{H}_{7} \mathrm{~F}_{4} \mathrm{~N}_{2}$

pa Chrg 1

3p:

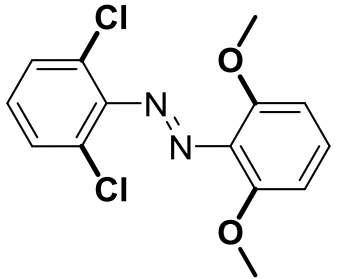

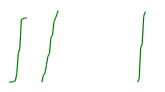
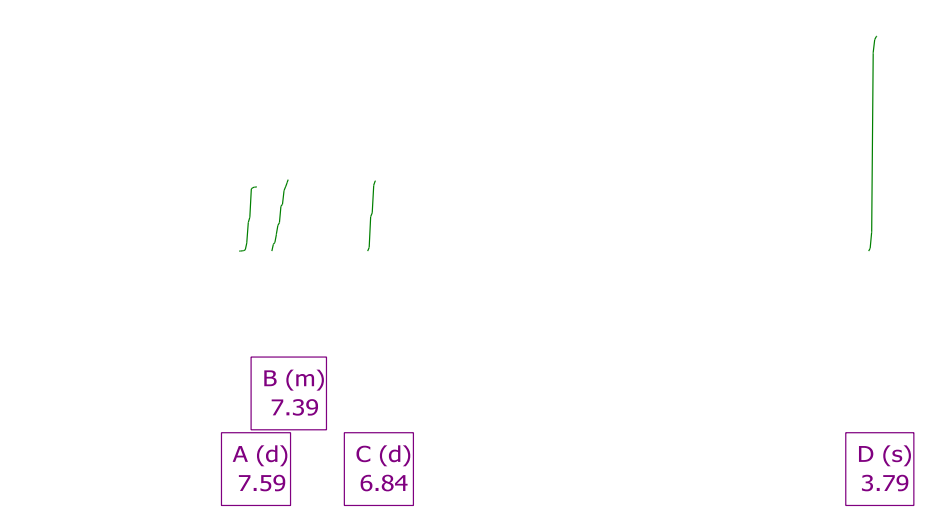
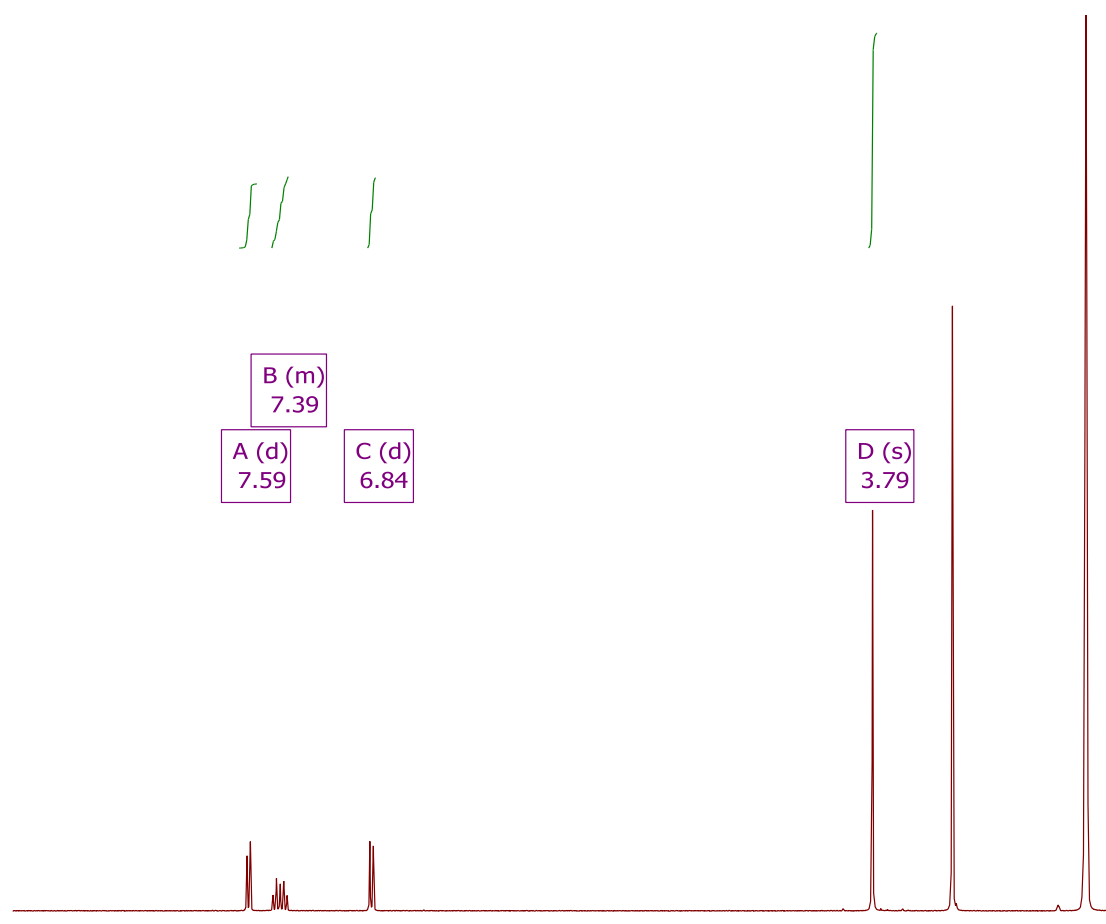

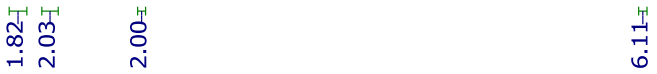

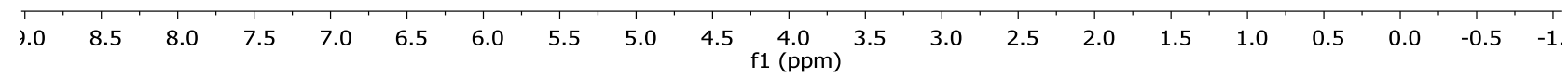




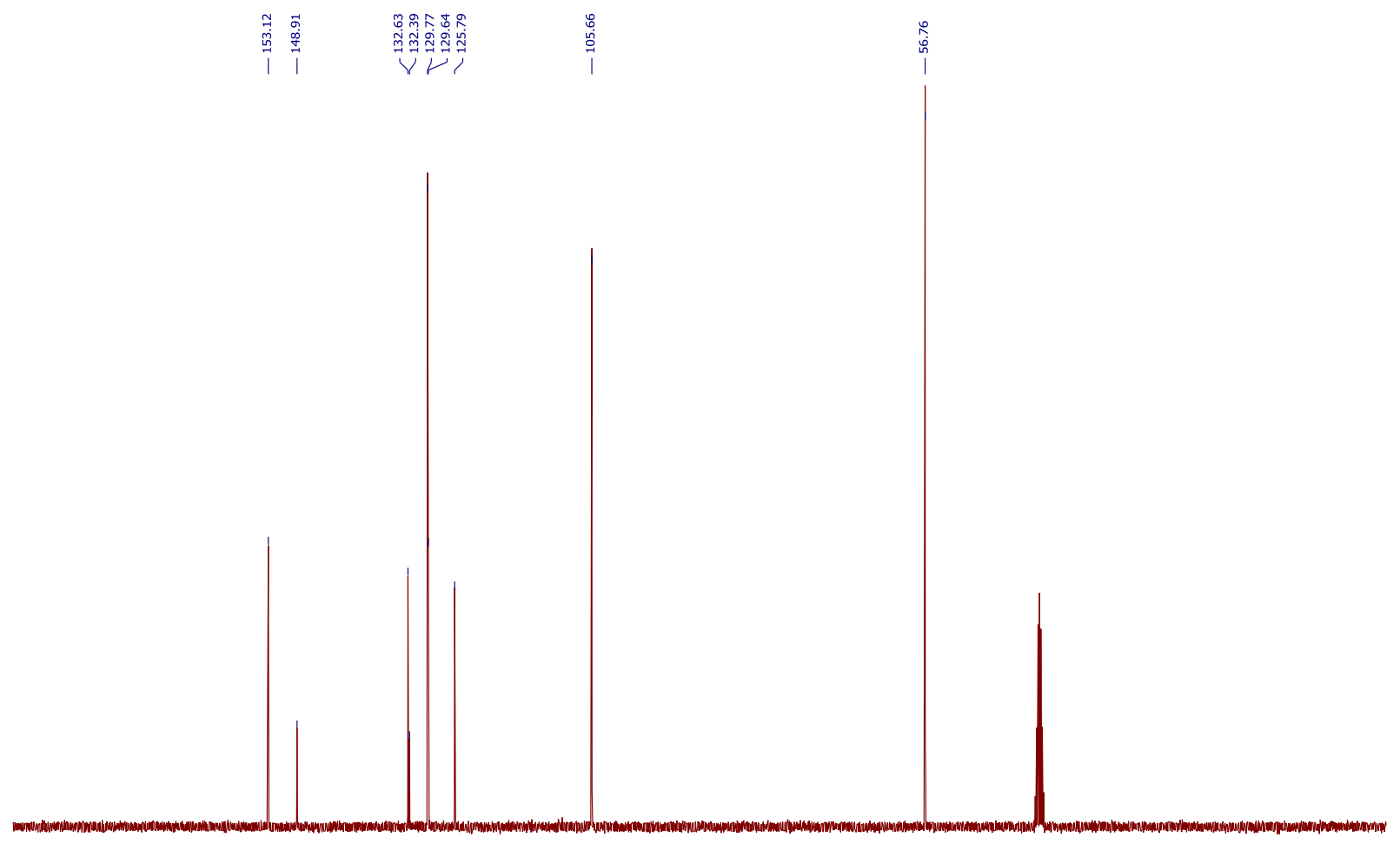

\begin{tabular}{|c|c|c|c|c|c|c|c|c|c|c|c|c|c|c|c|c|c|c|c|}
\hline 90 & 180 & 170 & 160 & 150 & 140 & 130 & 120 & 110 & 100 & $\begin{array}{l}90 \\
1(\mathrm{ppm})\end{array}$ & 80 & 70 & 60 & 50 & 40 & 30 & 20 & 10 & 0 \\
\hline
\end{tabular}

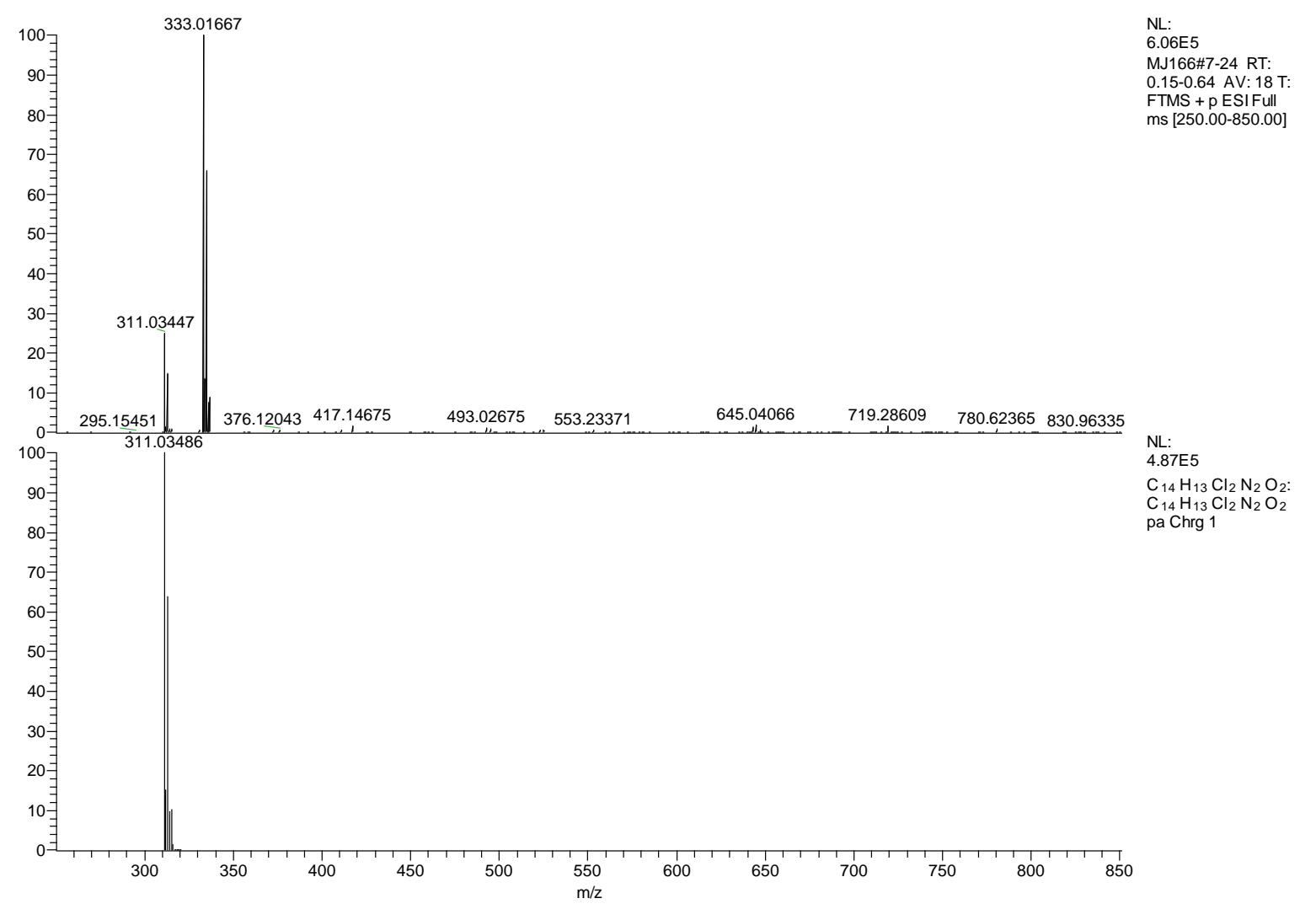


3q:
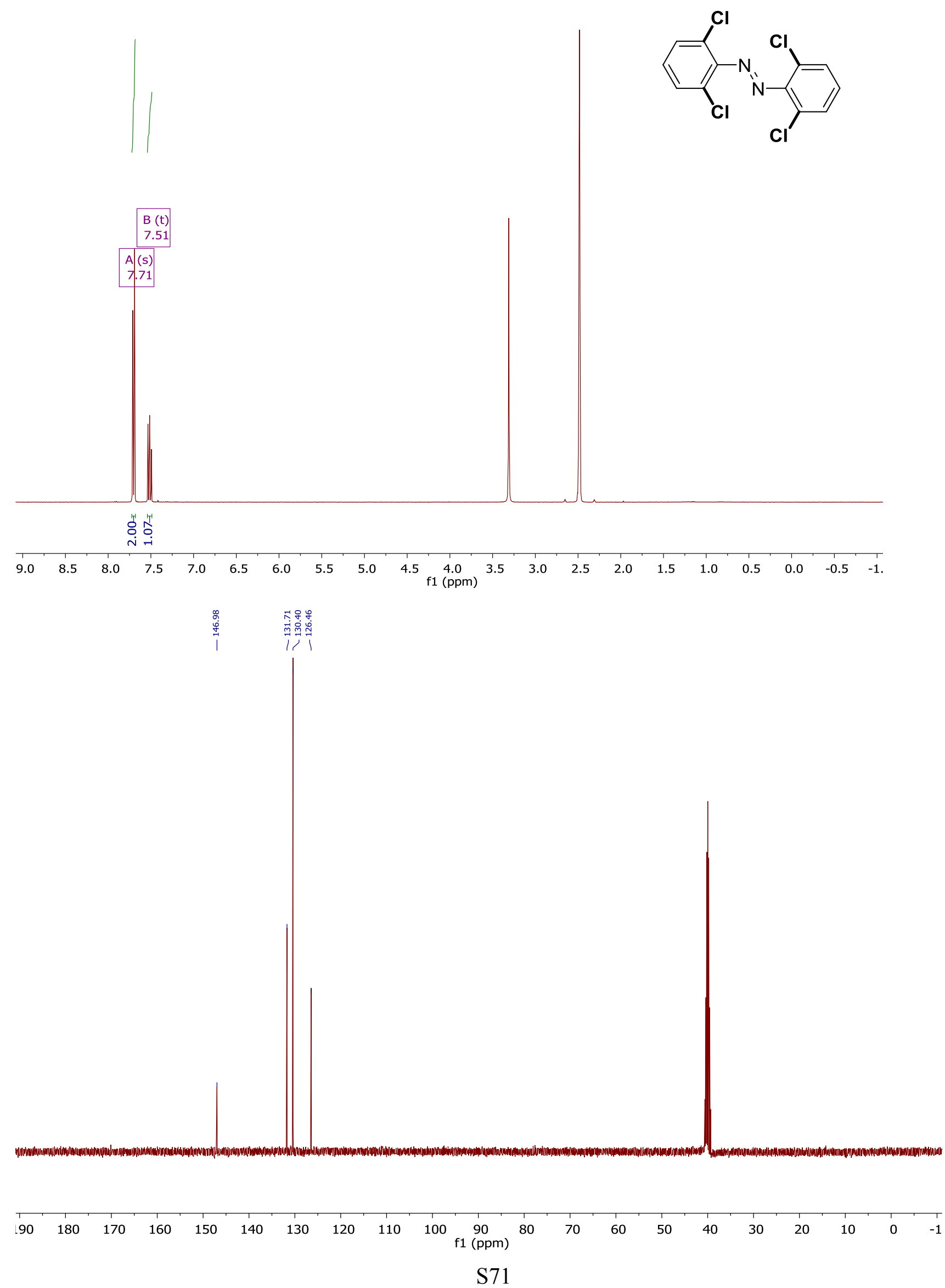


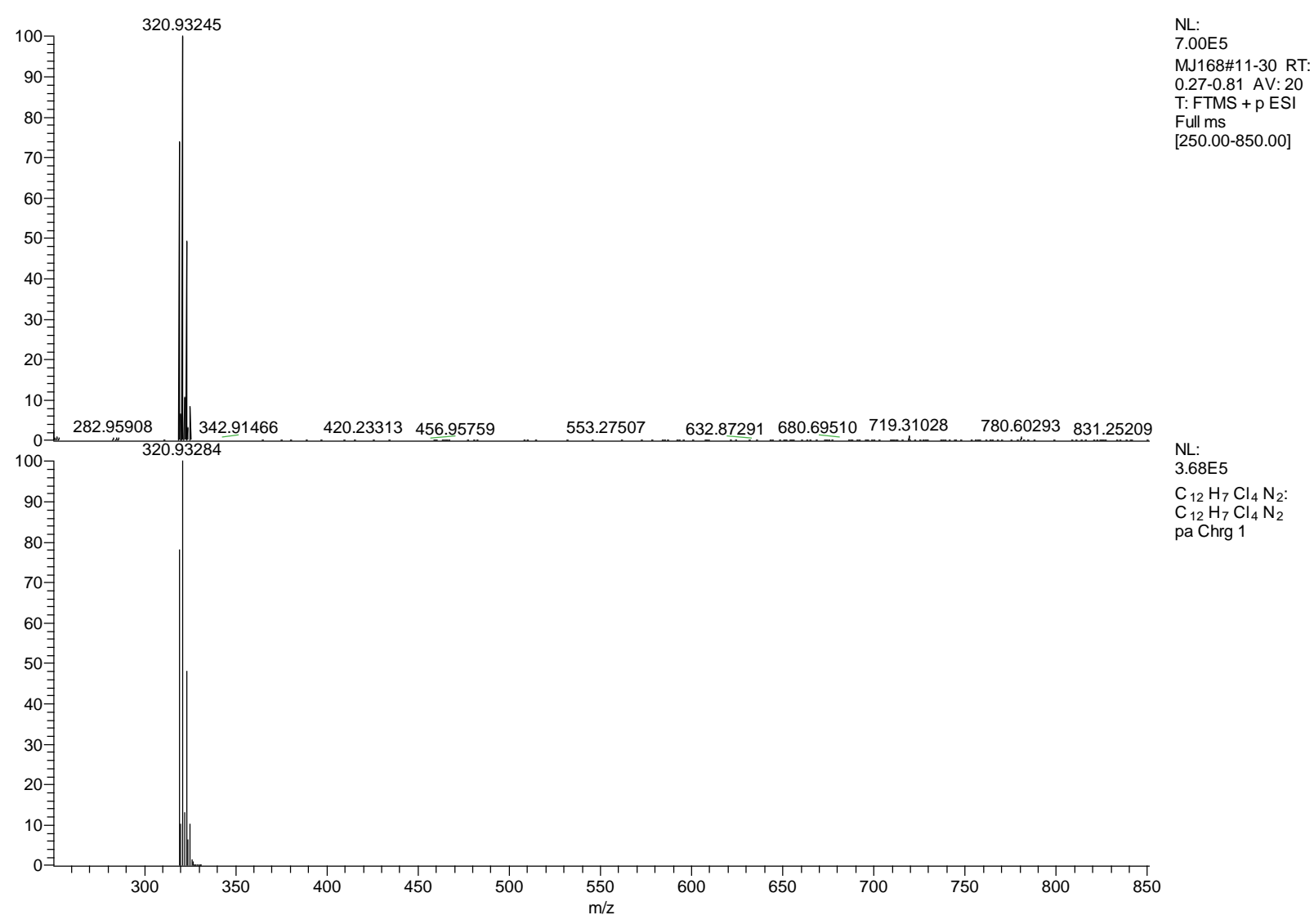

3r:

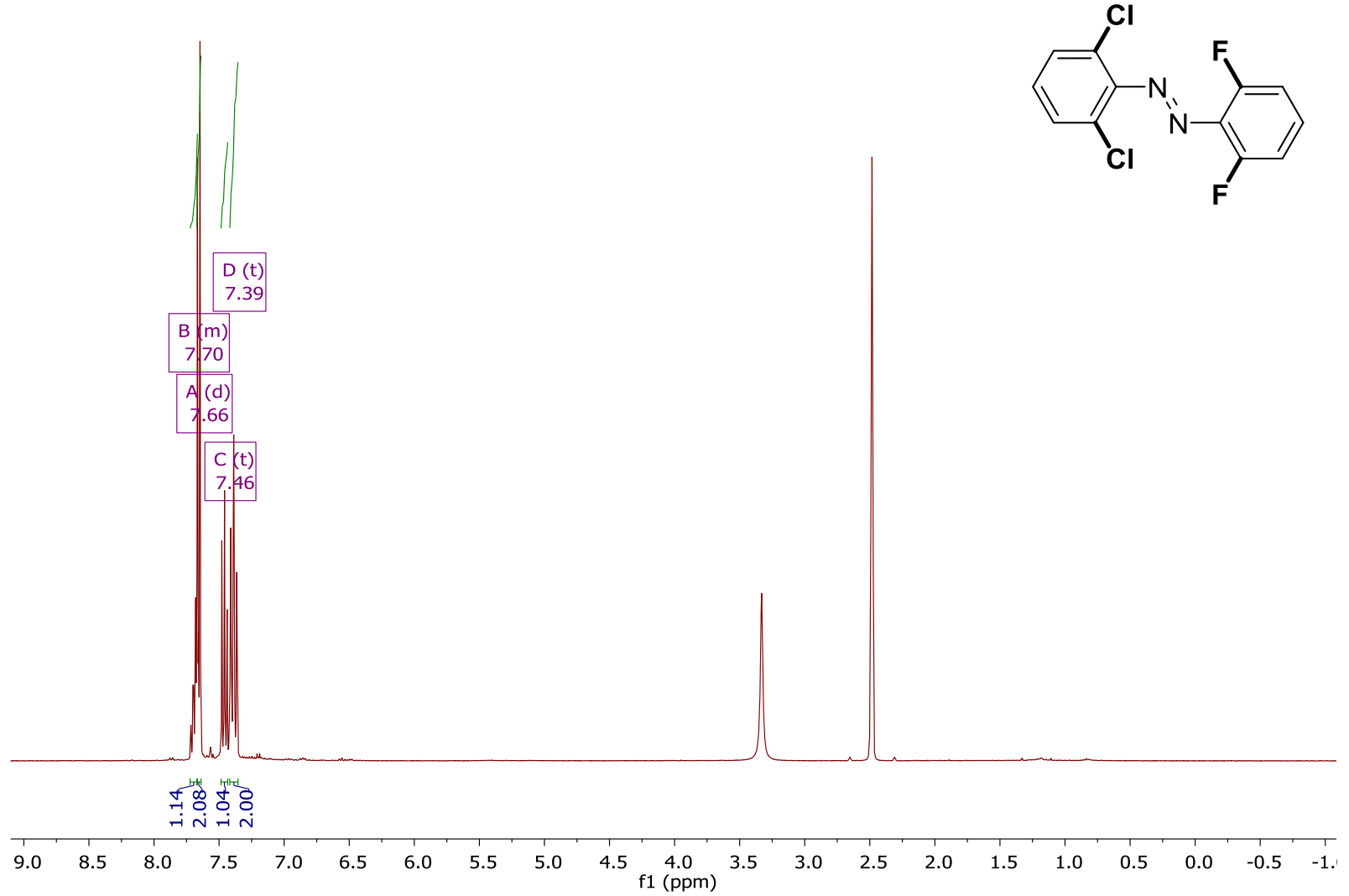



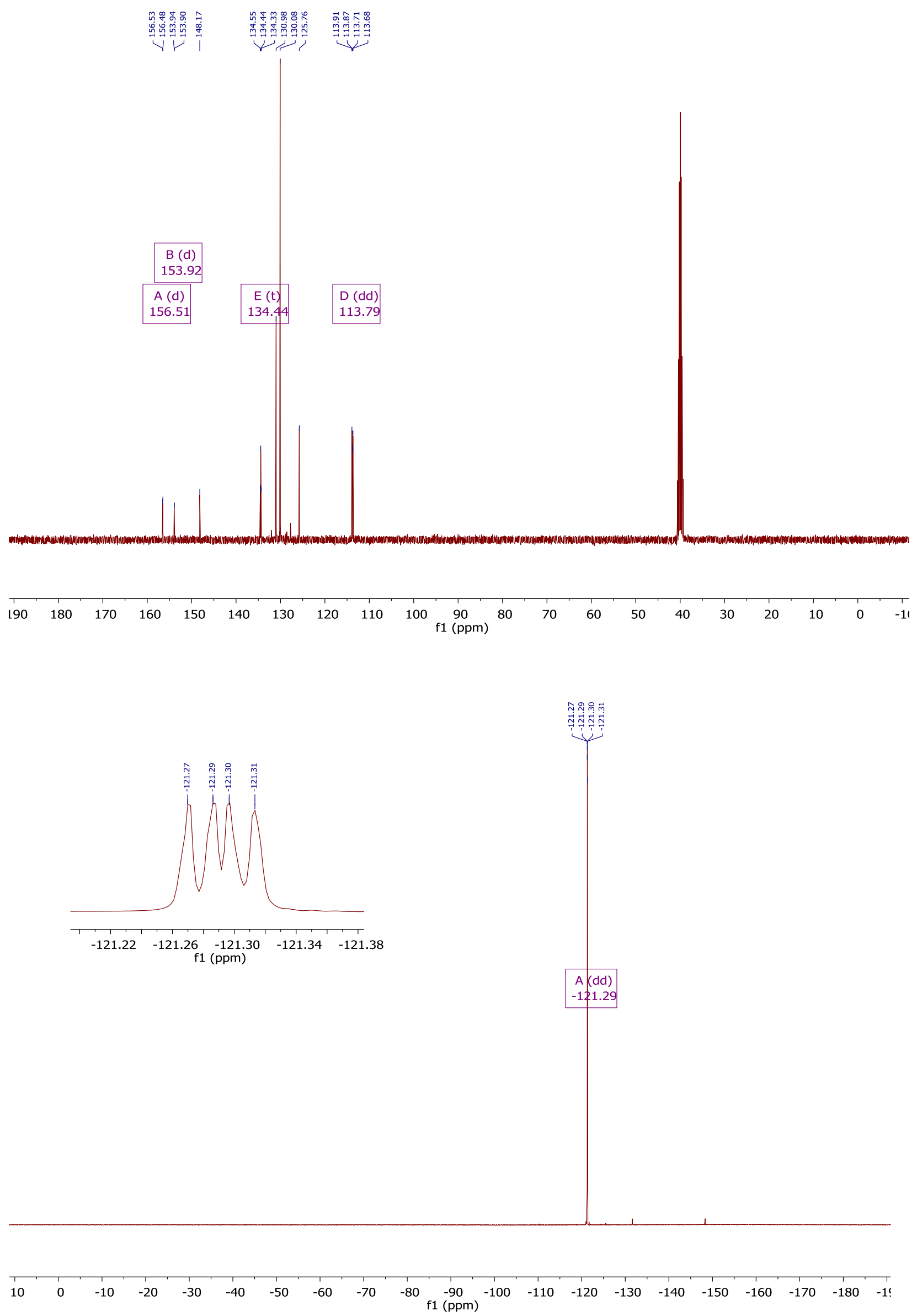

S73 


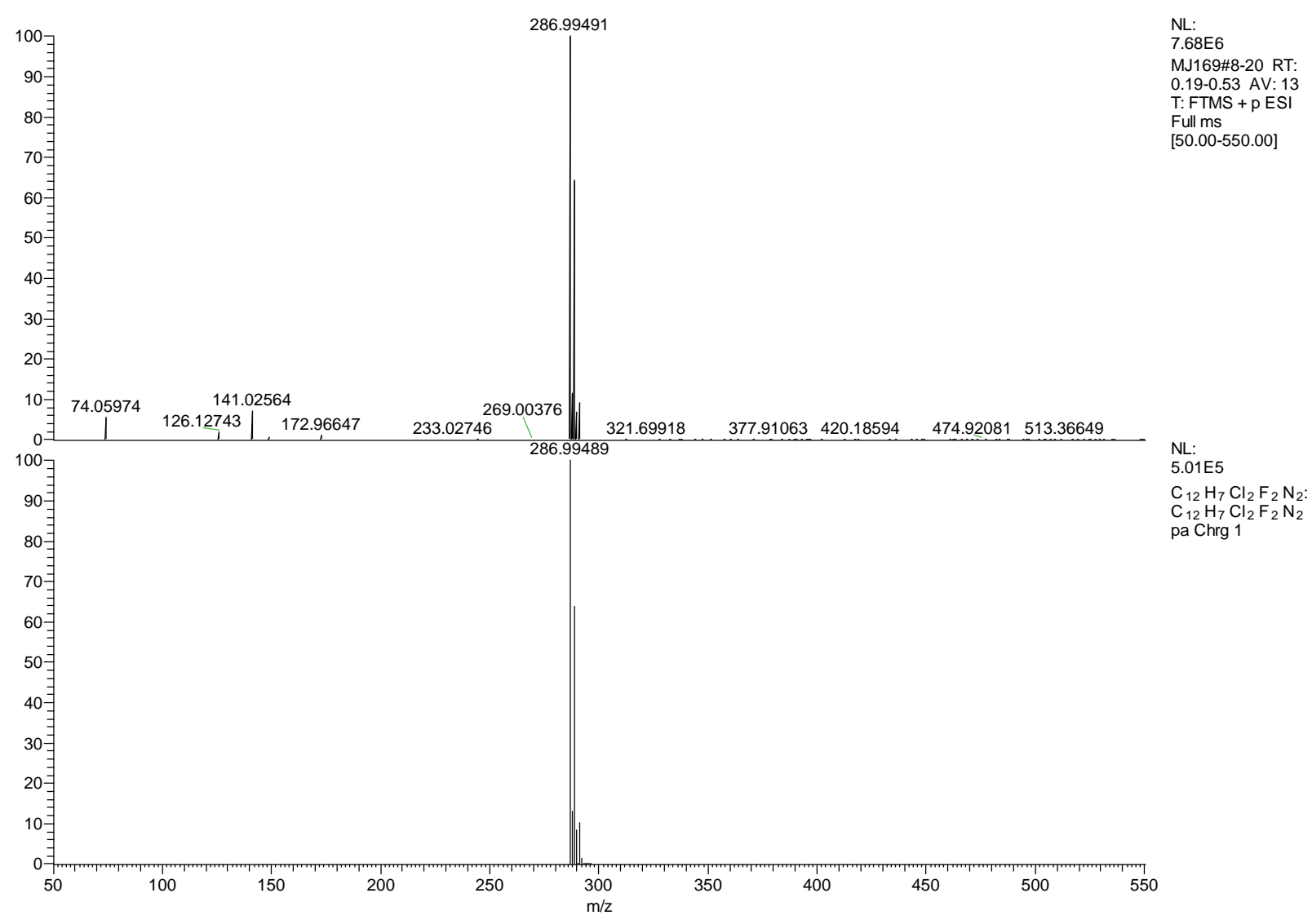

3s:

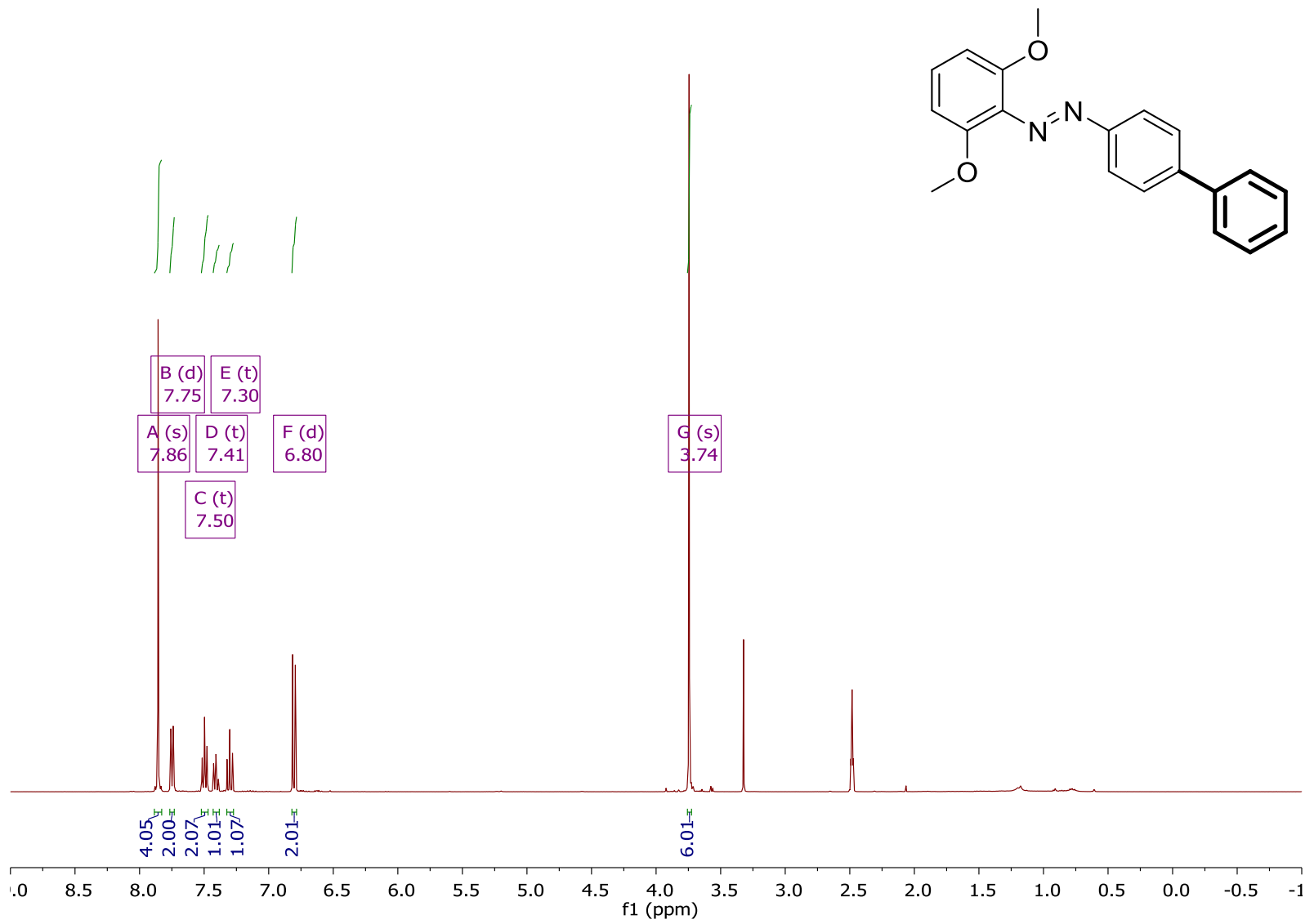



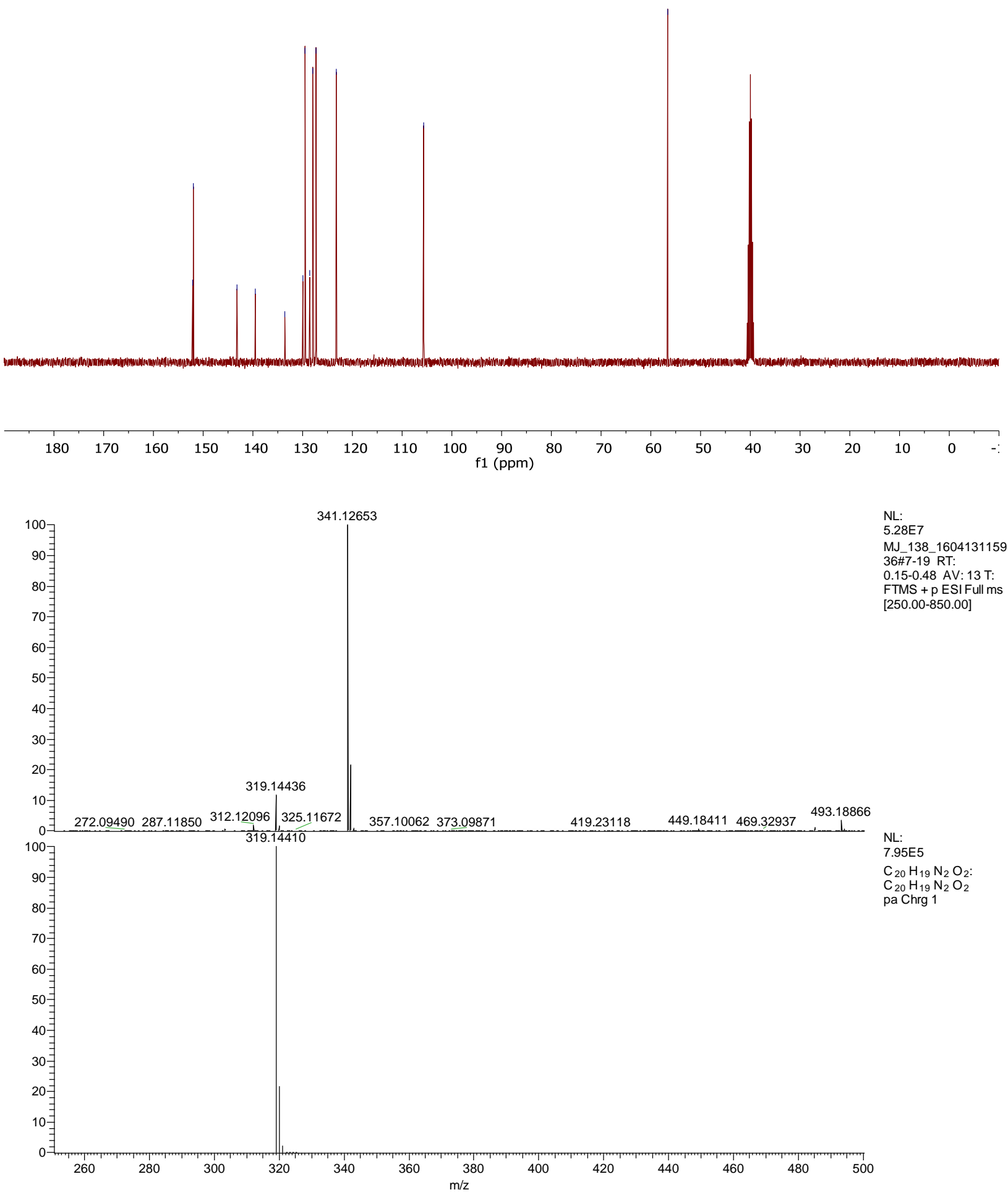


\section{References}

[1] P. S. J. Canning, K. McCrudden, H. Maskill, B. Sexton, J. Chem. Soc. Perkin Trans. 2 1999, 2735-2740.

[2] U. Wannagat, G. Hohlstein, Chem. Ber. 1955, 88, 1839-1846. 